, as

\title{
MASTER
}

\section{SEARCH FOR T-VIOLATION IN THE INELASTIC SCATTERING OF ELECTRONS FROM A POLARIZED PROTON TARGET}

Stephen Rock

(Ph.D. Thesis)

August 1970

AEC Contract No. W $-7405-$ eng -48
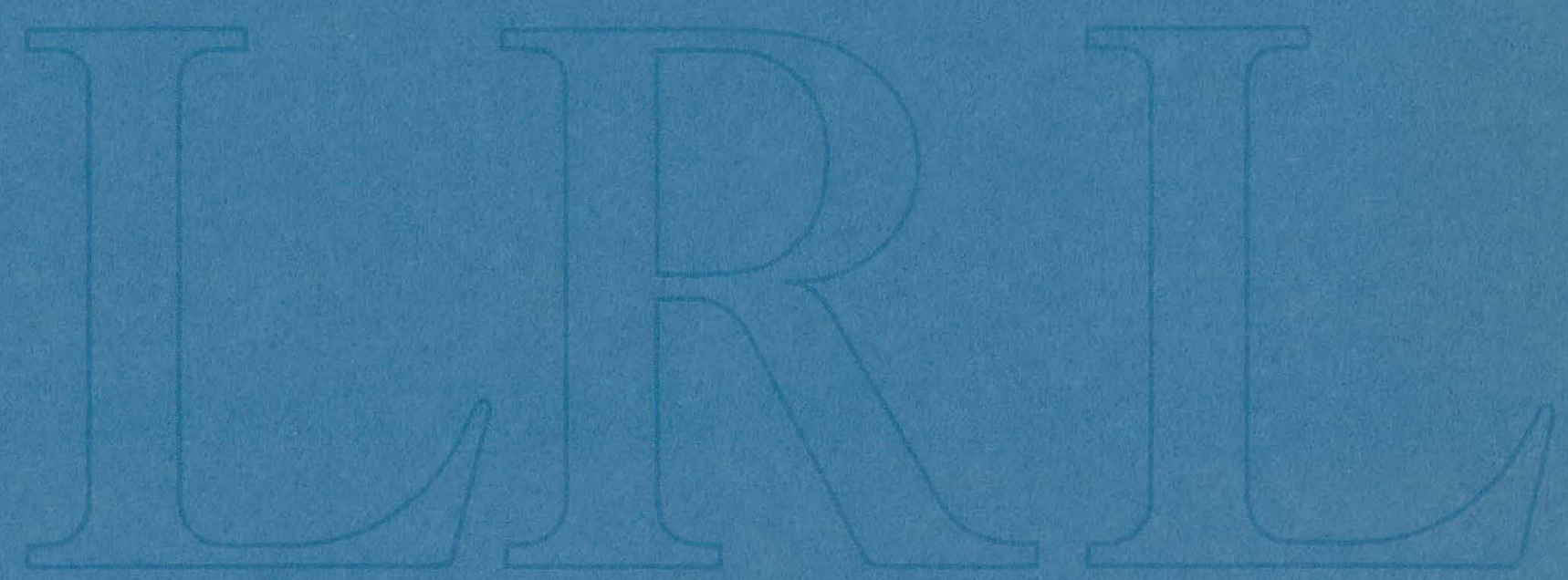

LAWRENCE RADIATION I.ABORATTORY UNIVERSITY of CAIIFORNIA BERKELEY 


\section{DISCLAIMER}

This report was prepared as an account of work sponsored by an agency of the United States Government. Neither the United States Government nor any agency Thereof, nor any of their employees, makes any warranty, express or implied, or assumes any legal liability or responsibility for the accuracy, completeness, or usefulness of any information, apparatus, product, or process disclosed, or represents that its use would not infringe privately owned rights. Reference herein to any specific commercial product, process, or service by trade name, trademark, manufacturer, or otherwise does not necessarily constitute or imply its endorsement, recommendation, or favoring by the United States Government or any agency thereof. The views and opinions of authors expressed herein do not necessarily state or reflect those of the United States Government or any agency thereof. 


\section{DISCLAIMER}

Portions of this document may be illegible in electronic image products. Images are produced from the best available original document. 


\section{PAGES $\mathrm{i}$ to $\mathrm{ii}$ WERE INTENTIONALLY LEFT BLANK}


Abstract. . . . . . . . . . . . . . . . . v

I. Introduction. .................. 1

II. Theory

A. History . . . . . . . . . . . . . . . 3

B. This Experiment ............... 6

C. Analysis of Kinematic Factors . . . . . . . . 21

D. Running Conditions. $+\cdots \cdot \ldots \cdot \cdots \cdot \cdots \cdot \cdots \cdot \cdots$

III. Experimental Design

A. Asymmetry Formula. . . . . . . . . . . 27

B. 'Errors and Target Polarization Reversal. . . . . . 30

C. Summary. . . ................ 33

IV. Experimental Setup

A. Beam. . . . . . . . . .... 34

B. Detection Equiprent . . . . . . . . . 38

C. Resolution. . . . . . . . . . . . 44

V. Polarized Target

A. Principles of Operation ............ 46

B. Choice of Material. . . . . . . . . . . 48

C. Target Preparation. . . . . . . . . . . 53

D. Cryostat. . . . . . . . . ... 56

E. Measurement of Target Polarization. . . . . . . . 64

VI. Running Procedure ....................

A. Beam Tune Up. . . . . . . . . . . . 72

B. Data Taking ................ 76 
VII. Analysis of Data

A. Least Squares Fit . . . . . . . . . . 79

B. Test Asymmetries. . . . . . . . . . 82

C. Deadtime................. 94

D. Monitors. ..................... 96

E. Regions . . . . . . . . . . . . . . 99

F. Selection of Data . . . . . . . . . . 100

VIII. Hydrogen Fraction . . . . . . . . . . . 104

IX. Results ... . . . . . . . . . . . . 109 Acknowledgments . . . . . . . . . . . . . . . I19 Dedication. . . . . . . . . . . . . 121 Appendices

A. Cancellation of Errors. . . . . . . . . 123

B. Kinematic Corrections . . . . . . . . . . 127

c. Target Hcating. . . . . . . . . . . 128

D. Statistical Independence of Asymmetries . . . . . . 132

E. Deadtime. . . . . . . . . . . . 134

F. Phony Bump. . . . . . . . . . . 135

References. . . . . . . . . . . . . 137 


\author{
$-\mathrm{V}-$ \\ SEARCH FOR T-VIOLATION IN THE INETASTIC SCATTERING OF \\ EIECTRONS FROM A POLARIZED PROTON TARGET \\ Stephen Rock \\ Lawrence Radiation Laboratory \\ University of California \\ Berkeley, California 94720
}

August 1970

\begin{abstract}
The recent discovery of a violation of $\mathrm{CP}$ invariance has led to speculation on the origins and nature of the symmetry violating forces. This experiment is a search for this violation in the electromagnetic interactions of hadrons. We looked for a violation of time-reversal invariance in inelastic electron scattering from a polarized proton target. A difference in cross sections (asymmetry) when the target was polarized in opposite directions along the normal to the scattering plane would mean either a time-reversal-invariance violation or a contribution from high order $\left(\alpha^{3}\right)$ effects. Data were taken using electron and positron beams to distinguish between the two possible causes of an asymmetry. Scattered electrons were detected in a magnetic spectrometer and the mass of the inferred outgoing hadronic state was calculated. The target polarization was reversed frequently in order to facilitate comparison of cross sections and to cancel random errors. The background from heavy unpolarized nuclei only contributed to an overall normalization factor. This was determined by supplementary measurements with carbon and $\mathrm{CH}_{2}$ targets. Data were taken at SLAC energies in the region of resonance excitation of the outgoing hadron and at values of four-momentum transfer squared of $0.4,0.6$, and 1.0
\end{abstract}


-vi-

$(\mathrm{GeV} / \mathrm{c})^{2}$. Our experimental errors were as small as $1.5 \%$ in asymmetry at certain kinematic conditions. No sizable violation of time-reversal invariance was found. 


\section{INT⿱RRODUCTION}

This is a report on an experimental investigation of the space-time symmetry of Time-Reversal Invariance (T) performed to help elucidate the problem of the CP violation. Over fifty years ago the discovery of the applications of statistics to large-scale phenomena and the symmetry under $T$ of classical mechanics and electrodynamics answered the questions of Time Reversal in macroscopic, ordinary phenomena. The basic questions, however, were left unsolved, only relegated to the level of atomic and nuclear sizies. Here, as in many other branches of science, we investigate submicroscopic phenomena with super-macroscopic equipment to find the answers to brain-size problems. Neither the extra-large (but far from economy size), nor the less-than-personal sizes involved should distract us from the physical and philosophical questions involved in whether it is possible to reverse processes and return them to their original state.

Our experiment is a test of Time-Reversal Invariance in the Electromagnetic Interaction of Hadrons, We scatter high energy electrons from a polarized proton target and detect the scattered electrons in a magnetic spectrometer. It is shown in section II that if there is a violation of Time-Reversal Invariance then there may be different cross sections when the target protons are polarized in opposite directions perpendicular to the plane of scattering (an asymmetry). The experimental procedure was to alternately measure the two cross sections by reversing the target polarization frequently without changing any other part of our apparatus. Corrections must be made for the unpolarized portion of the target: which will not contribute to an asymmetry. Special 
experimental efforts were made to avoid systematic and random errors and to minimize the damage to the polarized target due to the high intensity beam.

Part II of this paper describes the history and theory behind the experiment. Part III describes the phenomena as they appear in our experiment. The next two sections describe particular parts of the Gargantuan apparatus, IV the beam and detector, and $\mathrm{V}$ the polarized target and associated cryogenic equipment. Section VI describes how the experiment was actually operated. The Analysis of the Results begins in Section VII, where data reduction and possible sources of error are discussed, and concludes in VIII with a determination of the fraction of hydrogen in the target.

Results of this investigation and its interpretation are in the last section. We conclude that there is no evidence for a violation of Time-Reversal Invariance in the process we have studied within our experimental errors. However, the complexities of the big bad world are just reduced in scale, not in character, when one studies elementary particle phenomena. The reaction under study is so complicated that a T-violation could have taken sanctuary in some phase factor or cancellation ot' many terms. 


\section{$-3-$ \\ II. THEORY}

\section{A. History}

A symmetry operation in Physics is a transformation under which the appearance of the world (laws of physics) remain unchanged. Symmetries have long been viewed with extraordinary interest by both scientists and laymen because they reflect ideas of physical order, balance, simplicity, and beauty. The Greeks used the symmetry of the circle to great advantage in their astronomy, but clung to this unproven concept to the point where it limited the advance of knowledge. Most of the laws of classical physics are invariant under space-time symmetry operations. This gave an important scientific basis to the belief in symetries. Even twentieth century physicists had assumed that space inversion was a valid.symmetry (based on a plausable and beautiful, but unnecessary extension of the Lorenz group) until experimental evidence forced the opposite conclusion. Today we are more cautious about assuming the validity of symmetries and have more complex notions about what represents simplicity, yet high energy physicists are still more excited by the concepts of symmetry than by other recent developments. This is especially true of those symmetries closely connected with our basic macroscopic concepts of space-time.

The symmetry operators C (charge conjugation or particle antiparticle inversion), $P$ (parity or space inversion), and $T$ (time reversal) share these attractions. The symmetry of nature under the combined operation CPT is a consequence of special relativity and an extension of our macroscopic ideas of causality to elementary particle interactions. $^{1}$ CPT is thus an excellent illustration of the close connection 
between symmetry operators and our most fundamental beliefs on physical reality. We believe that any physical process and its CPT conjugate obey the same laws of physics. The CPT conjugate is one in which each particle is replaced by its antiparticle, spatial relationships are mirror inverted, and the process develops backward in time.

Experimental evidence on the validity of thcoc oymmctry opcrations is rather extensive now. ${ }^{2}$ In brief, $C$ has been tested in electromagnetic interactions of leptons and strong interactions of hadrons to a few percent, $P$ has been tested to about 1 in $10^{8}$ in electromagnetic interactions of leptons (atomic $\gamma$ transitions) and $I$ in $10^{5}$ in strong and electromagnetic interactions of hadrons (nuclear $\gamma$ transitions) and is violated maximally in weak interactions. T has been tested by reciprocity to about $1 / 2 \%$. The combination $\mathrm{CPT}$ has been tested to 1 in $10^{14}\left(\mathrm{~K}_{\mathrm{L}}, \mathrm{K}_{\mathrm{S}}\right.$ mass difference) and 1 in $10^{6}$ in the gyromagnetic ratio of electron versus positron and $\mu^{4}$ vs. $\mu^{-}$. Thus CPT in all three interactions and $P$ in the electromagnetic and strong interactions have been tested to many orders of magnitude better then $\mathrm{C}$ or $\mathrm{T}$.

Since these operators have direct physical meaning, as do translations and rotations, one can put physical restrictions on these uperators. For example: space inversion $\mathrm{R}$ should commute with Time Translation. In quantum mechanical terminology the Tirne Translation operator $=\exp (\mathrm{iHt})$ where $H$ is the Hamiltonian and thus

$$
R \cdot \exp (i H t)=\exp (i H t) \cdot R \text { therefore }[H, R]=0
$$

or Nature is invariant under space inversion. This relationship aroused no interest until 1956 when Lee and Yang ${ }^{3}$ suggested and Wu, Amber, et al. 4 proved experimentally that parity was no longer a valid symmetry so 
$[\mathrm{H}, \mathrm{P}] \neq \mathrm{O}$ in the weak interactions. However if one naturally associates $\mathrm{R}=\mathrm{P}$ as was normally done, a contradiction results. The expedient solutions was to associate $R=C P$ since $C P$ was, at that time, considered to be a valid symmetry and numerous tests were done to show this such as studies of $\mu \rightarrow$ evv. Since $C$ has no effect on space coordinates, $C P$ on space is the same as $\mathrm{P}$ on space; therefore this view seemed generally acceptable.

However in 1964, when Christenson et al..$^{5}$ discovered that CP was not a valid symmetry in $K_{L}$ decay, the paradox reasserted itself and remains unexplained. Because CPT invariance seems to be essential to a relativistically satisfactory theory we infer that the observed $C P$ violation means that $T$ violations will likely appear somewhere, as we shall now see. 
B. This Experiment

As of now there is no convincing explanation for the CP violation and the experiment described in this paper is one of the many designed to shed light on the problem. We have made a test of the theory of Bernstein, Feinberg, and Lee $(\mathrm{BFL})^{6}$ that the $\mathrm{CP}$ violation is due to the electromagnetic current of hadrons which has equa.77y 7arge GP sonserving and $\mathrm{CP}$ violating parts (maximal violation, just as the weak current has equally large $\mathrm{P}$ conserving and $\mathrm{P}$ violating parts). The inspiration for this is the size of the CP violating term in $K_{L}$ decay $\left(2 \times 10^{-3}\right.$ in amplitude) which is of the order of a virtual electromagnetic correction $\alpha / \pi$. BFI pointed out that the experimental evidence cited above made no statements as to the $\mathrm{C}$ and $\mathrm{T}$ operators in the electromagnetic interactions of hadrons. It was postulated that each type of interaction (strong, $E$ \& $M$ and weak) has its own symmetry operator (e.g. $\mathrm{C}_{\mathrm{st}}, \mathrm{C}_{\gamma}$, and $\mathrm{C}_{\text {wk }}$ ) under which they are separately invariant, but that one type of interaction is not necessarily invariant under the symetry operators of another. Thus $\mathrm{P}_{\text {st }}=\mathrm{P}_{\gamma}$ from experimental evidence cited above (nuclear $\gamma$ transitions) but the fact that $\mathrm{P}_{\text {st }} \neq \mathrm{P}_{\text {wk }}$ is what is meant by the Parity (meaning $\mathrm{P}=\mathrm{P}_{\text {st }}=\mathrm{P}_{\gamma}$ ) violation in weak interactions. Similarly if $\mathrm{C}_{\text {st }} \neq \mathrm{C}_{\gamma}$ ( $\mathrm{C}_{\text {st }}$ is particle $\leftrightarrow$ anti-particle; $\mathrm{C}_{\gamma}$ is charge $\leftrightarrow$-charge) then there would be an apparent violation of $\mathrm{C}=\mathrm{C}_{\text {st }}$ and $\mathrm{CP}=\mathrm{C}_{\text {st }} \mathrm{P}$ in the electromagnetic interactions of hadrons. Since CPT is assumed valid to high

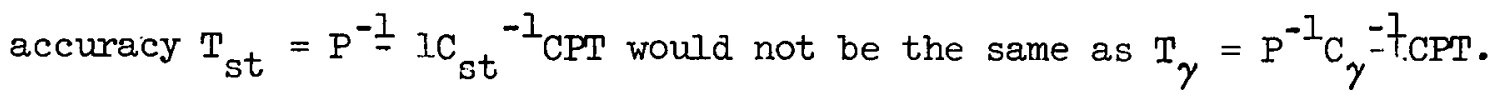
Thus to test Bernstein, Feinberg, and Lee's theory one looks for $\mathrm{C}$ or $\mathrm{T}$ noninvariance in the electromagnetic interactions of hadrons. This formulation of the $C, P, T$.symmetries limits the paradox discussed above 
by replacing $H$ with the appropriate interaction Hamiltonian $H_{i}$ and $R=P_{i}$ defined by the same interaction.

Since this suggestion in 1965, numerous experiments have been carried out to test the hypothesis. For example the electric dipole moment of the neutron, which should vanish due to $\mathrm{T}$ invariance has been measured ${ }^{2}$ to be $\leq 10^{-23}$ e-cm with predictions changing to fit the data. C noninvariance would create an asymmetry in the momentum distribution of $\pi^{+} \pi^{-}$in $\eta \rightarrow \pi^{+} \pi^{-} \pi^{0}$ which is predicted to be a few percent ${ }^{7}$ and the latest experimental results ${ }^{8}$ of $(1.66 \pm 0.63) \%$ are far from conclusive. The BFL theory had been neither confirmed nor convincingly disproved as of this experiment.

Christ and Lee $^{9}$ showed that an excellent test for BFL theory would be a measurement of the asymmetry in inelastic electron scattering from a polarized proton target. This asymmetry is the relative difference in cross sections for an all proton target polarized completely parallel and anti-parallel to the scattering plane, i.e.

$$
A=\frac{\sigma_{\uparrow}=\sigma_{\downarrow}}{\sigma_{\uparrow}+\sigma_{\downarrow}}
$$

Any asymmetry in this electromagnetic interaction of hadrons would be a violation of $T$ invariance (in the one-photon-exchange approximation) and for maximal violation a large polarization $\sim 30 \%$ is expected. A preliminary experimental measurement of this asymmetry has been made by Chen, et al. ${ }^{10}$ other experiments are not expected to have as large a potential effect as this one. BFL's theory is in principle easier to test than others because it predicts equal CP violating and non-violating amplitudes in electromagnetic interactions of hadrons. Other theories 
have small $\left(<_{10}^{-3}\right) \mathrm{CP}$ violating amplitudes compared to non-violating and hence one must do a very careful experiment to observe the violation. Unfortunately most tests of the BF' hypothesis involve a reduction of the expected violation due to kinematical or other considerations. For example in the above mentioned $\eta \rightarrow 3 \pi, \eta$ has $J=0$ and $C P=-1$. The $C P$ conserving system has the same values of $J$ and $C P$ and so in the most probable state all orbital angular momenta are 0 and $I=1$ to make the system symmetric. The $\mathrm{CP}$ violating decay then goes into a $\mathrm{CP}=+3 \pi$ system which must have at least $L_{\pi^{+}, \pi^{-}}=1$ and $L_{\pi^{+} \pi^{-}, \pi^{0}}=1$ to have $\mathrm{J}^{\mathrm{CP}}=\mathrm{O}^{+}$. To be symmetric this system must be $\mathrm{I}=0$. The two orbital angular momenta reduce the CP violating amplitude by an angular momentum barrier $\sim(\mathrm{kr})^{6}$ with an unknown interaction radius $r$. Putting in a "reasionabie" value of $r$ one gets a possible charge asymmetry of only a few percent despite maximal violation. ${ }^{\phi}$ similar suppresoions exist in other possible tests. The $\mathrm{e}-\mathrm{p}$ inelastic scattering is free from these types of suppression and thus high polarizations are expected with a CP violating electromagnetic interaction.

It is not obvious that Time Reversal Invariance forbids an asymmetry (in the one-photon-exchange approximation) and two proofs will be given emphasizing the specific form of the interaction and the nther the generality of the proof. Note that both depend on the interaction being first order in $\alpha$ and neglecting higher order terms (Feynman diagrams). The effect of the higher order terms will be discussed later. The only Feynman diagram of first order is shown in Fig. la. Theorem: 'l'here is no asymmetry in inelastic electron scattering from a polarized proton target in the one-photon-exchange approximation, if 
initial electron spin is averaged and all final spins are summed over and Time-Reversal Invariance holds.

Proof I: By the laws of quantum electrodynamics the interaction Hamiltonian represented by the diagram of Fig. la is a product of two currents $\mathrm{H}=g_{\mathrm{u}}^{\ell} g_{\mathrm{u}}^{\mathrm{h}}$; where $f_{\mathrm{u}}^{\ell}$ is the well-known leptonic electromagnetic current $e \bar{u} \gamma_{\mu} u=\mathcal{f}_{u}^{\ell}$ and $\ell_{u}^{h}$ is the hadronic electromagnetic current which is unknown, but can be written down ${ }^{11}$ in the most general form (consistent with special relativity) in terms of a priori unknown but experimenta,jy determinable form factors. The only fact important to the proof is that both currents are of magnitude $e$ and thus $H \alpha e^{2}=\alpha$ which is small. The Hamiltonian can be related to the $\mathrm{S}$ matrix in the usual perturbation expansion derived from the Schrodinger equation

$$
\text { i } \frac{\partial \psi}{\partial t}=H \psi(t)
$$

and the time development operator U:

$$
\psi(t)=U\left(t, t_{0}\right) \psi\left(t_{0}\right)
$$

$H$ and $U$ are thus related by:

$$
U\left(t, t_{0}\right)=1-i \int_{t_{0}}^{t} H\left(t^{\prime}\right) U\left(t^{\prime}, t_{0}\right) d t^{\prime}
$$

and to lowest order in $\boldsymbol{\alpha}$ :

$$
u\left(t, t_{0}\right)=1-i \int_{t_{0}}^{t} H\left(t^{\prime}\right) d t^{\prime}
$$

The $S$ matrix is

$$
\begin{aligned}
& \operatorname{limit.}_{t \rightarrow+\infty} \quad \mathrm{T}\left(t_{1}, t_{0}\right) \\
& t_{0} \rightarrow-\infty
\end{aligned}
$$




\section{Feynman diagrams}

(a)

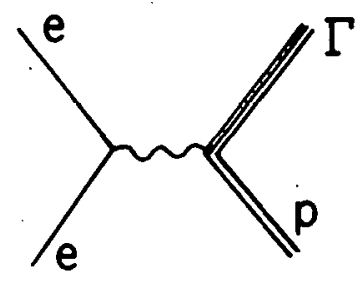

(b)

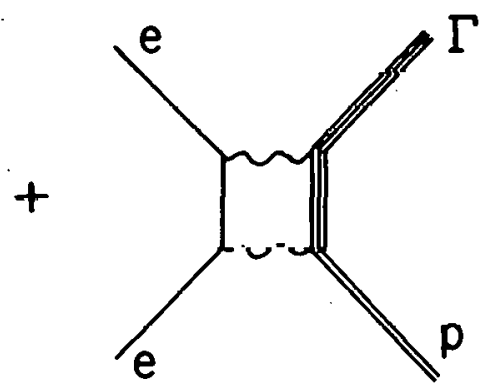

(c)

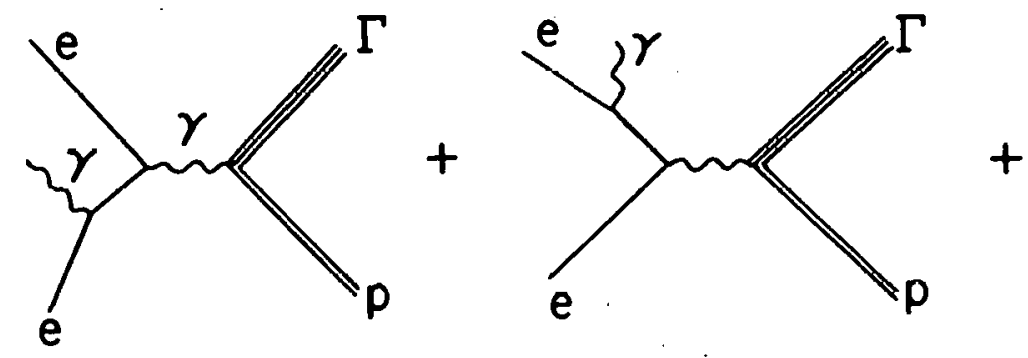

(d)
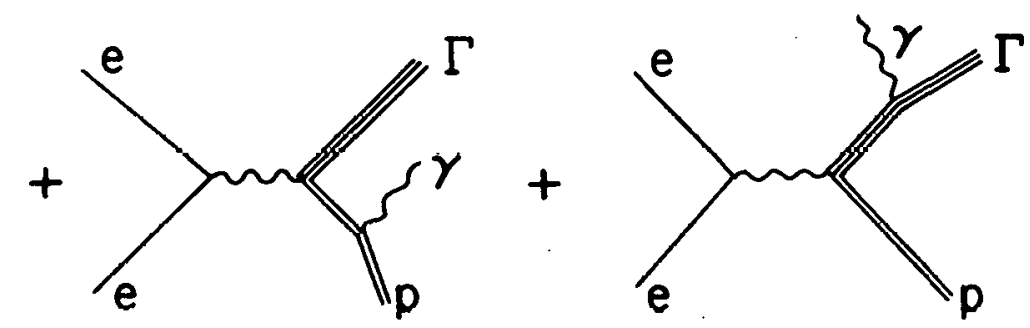

XBL 706-3200

Fig. 1. Feynman diagrams for the process ep $\rightarrow \mathrm{e} \Gamma$

a) The one-photon-exchange process

b) The two-photon-exchange process

c) Radiative diagrans with photon on electron line

d) Radiative diagrams with photon on hadron line Note that the second diagram in 1.d. can be altered to become 1. a by redefining $\Gamma$ to include an extra photon. $l a$ and $l b$ interfere as do $I c$ and $l d$. 
hence

$$
S=I-i \int_{-\infty}^{\infty} H\left(t^{\prime}\right) d t^{\prime}
$$

The usual expansion of $\mathrm{S} \cdot$ is:

$$
S=1+i M
$$

where $M$ is the transition matrix element. Since the Hamiltonian is Hermitian so is $M$ and thus

$$
M_{f i}=M_{i f}^{*}
$$

Time Reversal invariance means that

$$
M_{f i}=M_{i} t_{f} E
$$

where the superscript $t$ means time-reversed state, therefore spin and momenta are reversed. Thus the matrix element for a process going one way is the same as the matrix element of the process going the other way with spins and momenta reversed. Combining both relations

$$
M_{f i}=M_{i f}^{*}=M_{f}^{*} t_{i} t
$$

and taking absolute squares to get cross sections (and suppressing some numerical factors):

$$
\sigma_{f i}=\left|M_{f i}\right|^{2}=\left|M_{f}^{*} t_{i}\right|^{2}=\sigma_{f t_{i} t^{i}}
$$

If one quantizes the spin perpendicular to the plane of the interaction a single rotation as shown in Fig. 2 restores the momenta in a state $j^{t}$ to that of state $j$ without affecting the spin. Figure $2 a$ shows the process $i \rightarrow f$, Fig. $2 b$ the process $i^{t} \rightarrow f^{t}$. A rotation of $180^{\circ}$ about the normal to the scattering plane restores the momenta to their original values leaving the spins, as shown in Fig. 2c. Let a superscript $s$ 
a) Reaction: $\frac{\operatorname{Spin} \odot}{e}$

b)
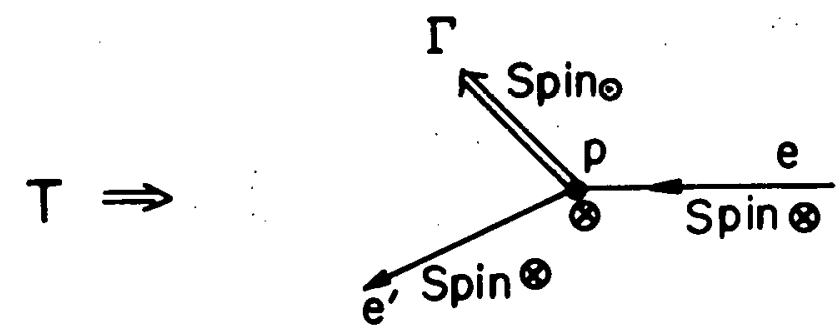

c) $R\left(180^{\circ}\right) \Rightarrow \frac{e}{\operatorname{spin} \otimes}$

XBL706-3202

Fig. 2. a) The reaction ep $\rightarrow \mathrm{e} \Gamma$ in the laboratory, showing the spins and directions of the particises.

b) The rcaction after the operator $T$ has been applied. c) The reaction after a rotation of $180^{\circ}$ about the normal to the scattering plane has been performed. 
represent the state after this process (namely just the spins reversed). Then

$$
\sigma_{f i}=\sigma_{f^{\dot{s}} i \dot{s}}
$$

If we sum over all final-state spins, the sum over $f$ is the same as that over $f^{\mathrm{s}}$ and so

$$
\sum_{f} \sigma_{f i}=\sum_{f} \sigma_{f i s} .
$$

Averaging over the initial electron spins results in the equality of the cross sections with proton spins oppositely polarized in the normal to the scattering plane with all other conditions the same.

Proof II: We use the Unitarity relation $S^{\dagger} S=1$, in the form $M^{\dagger}=S^{\dagger} M$, which can be expressed as

$$
M_{i f}^{*}=\sum_{n} S_{n f}^{*} M_{n i}
$$

where $\mathrm{n}$ is any physically realizable intermediate state. To conform to the experimental setup described below we define a set of states G which are restricted only by being physically realizable from the state of incoming electron and target proton and having the final electron coming out at a fixed angle and energy. The states $G$ are what we detect experimentally and so a sum over all states $f \in G$ constitutes the cross section we are measuring

$$
\begin{aligned}
& \sum_{f \in G}\left|M_{i f}^{*}\right|^{2}=\sum_{f \in G} \sum_{n_{, n^{\prime}}} S_{n f}^{*} M_{n_{i}} S_{n^{\prime} f} M_{n^{\prime} i}^{*}= \\
& =\sum_{n n^{\prime}} M_{n i} M_{n^{\prime} i}^{*} \sum_{f \in G} S_{n f}^{*} S_{n^{\prime} f}
\end{aligned}
$$


$-14-$

Now we use the one- $\gamma$-exchange approximation to get the magnitudes of $M$ and $S$ in order to determine which terms can be ignored. Since the electron and proton can only interact by exchanging at least one photon $\mathrm{M}_{n i}=\theta\left(e^{2}\right)$ to any physical state $\mathrm{n}$. Also, since $\mathrm{S}_{\mathrm{ab}}=\dot{\delta}_{\mathrm{ab}}+\mathrm{iM}_{\mathrm{ab}}$, $S_{a b}=0\left(e^{2}\right)$ if $a \neq b$. The one- $\gamma$-exchange approximation allows one to ignore all terms in the cross section of order greater then $e^{4}$ or $e^{2}$.

To evaluate (II-2) divide the sum over $n$ and $n^{\prime}$ into two different categories: 1) $n$ and $n^{\prime} \in G$; 2) the rest. Thus

$$
\sum_{f \in G}\left|M_{i f}^{*}\right|^{2}=\sum_{(1)}\left[M_{n i} M_{n^{\prime} i}^{*} \sum_{f \in G} S_{n f}^{*} S_{n^{\prime} f}\right]+\sum_{(2)}[]
$$

Sum (2) is of order $\alpha^{3}$ since one or both of the $S$ terms is of order $\alpha$ (since $f \in G$ and either or both $n, n^{\prime} \in G$ ) and both $M$. terms are of order $\alpha$. Thus we can drop sum (2). The sum over fid. is almost represented by

$$
I=S^{\dagger} S=\sum_{\text {alt } f} S_{n^{\prime} f} S_{n f}^{*}=\delta_{n n^{\prime}}=\sum_{f \in G} S_{n f}^{*} S_{n^{\prime} f}+\sum_{f \notin G} S_{n f}^{*} S_{n^{\prime} \epsilon}
$$

Hence:

$$
\sum_{f \in G}\left|M_{i f}^{*}\right|^{2}=\sum_{n, n^{\prime} \in G} M_{n i} M_{n^{\prime} i}^{*}\left(\delta_{n n^{\prime}}-\sum_{f \notin G} S_{n f}^{*} S_{n^{\prime} f}\right)
$$

The second term is of order $\alpha^{4}$ for the same reason as above and hence

$$
\sum_{f \in G}\left|M_{i f}^{*}\right|^{2}=\sum_{n \in G}\left|M_{n i}\right|^{2}=\sum_{f \in G}\left|M_{f i}\right|^{2}
$$

Now (at last) we use time-reversal invariance in the dame form as above

$$
\mathrm{M}_{\mathrm{ab}}=\mathrm{M}_{\mathrm{b}} \mathrm{t}_{\mathrm{a}} \mathrm{t}=\mathrm{M}_{\mathrm{b}} \mathrm{s}
$$

Hence: $\sum_{f \in G} \sigma_{f i^{s}}=\sum_{f \in G} \sigma_{f i}$

QED 
This second proof has the aesthetic advantage of not using any particular form for the interaction except that it is electromagnetic.

Both proofs (and in fact all proofs) depend on 1) The assumption of $1-\gamma$ exchange, 2) Summing over final states. The first proof indiates we only have to sum over (detect) all final spins of a particular state, while the second proof, with fewer assumptions, requires a uniform detection of all final states with fixed electron energy and angle. Since the decay angular distribution of a resonance depends on its spin it is essential that we detect all possible directions of the final particles with equal efficiencies, which is easiest done by detecting none of them, i.e. only detecting the scattered electron.

If either (1) or (2) were violated, a n asymmetry could exist without violating $T$ invariance. Luckily we can tell the difference between an asymmetry caused by $\mathrm{T}$ non-invariance and one caused by higher order corrections to the one-photon-exchange diagram. As shown in Figs. $l a$ and $l b$, the one- $\gamma$-exchange diagram has one power of $e$, the lepton charge, on the lepton line. When the matrix element is squared it becomes $e^{2}$, independent of the charge of the lepton. However the two$\gamma$-exchange diagram contributes most in the interference term of the product of one- $\gamma$ - and two- $\gamma$-exchange diagrams. Here there is a factor of $e^{3}$ which does change sign with lepton charge. Thus, doing the experiment with both $e^{-}$and $e^{+}$and looking for a change in sign of asymmetry allows one to distinguish between $T$ violation and two- $\gamma$ exchange. The two-photon-exchange contribution is expected to be of order $\alpha<1 \%$ and thus not likely to show up within our experimental accuracy of about $1-1 / 2 \%$. 
There are other higher order diagrams which must be considered because of their rather large effect on the cross section. These are the "radiative". diagrams shown in Figs. Ic and ld which have a soft $\gamma$ in the final state. Our detector, which only accepts the electron, cannot distinguish between the non-radiative and radiative interactions (no interaction is truly non-radiative since there are an infinite number of zero energy photons always emitted). Clearly in the limit of zero photon energy the radiation can have no effect on our calculations and introduces no asymmetry. However, even though the radiative diagrams are higher order in $\alpha\left(\alpha^{3}\right)$ there are so many of them contributing to different values of photon momentum that they make a significant contribution to the cross section and thus might make a contribution to an asymmetry. Cahn and Tsai ${ }^{12}$ have shown that if one considers only diagrams where the bremsstrahlung is from the lepton lines, Fig. lc, then these do not contribute to an asymmetry if $\mathrm{T}$ invariance holds. Diagrams where the bremsstrāhlung is from the hadron line, Fig. Id, and interference between the two types can contribute to an asymmetry, but these are very small due to the hïgher mass of the proton. This is confirmed by the calculations of Cahn and Tsai on such an interference which yields $\mathrm{A} \ll 1 \%$.

In cross-section measurements one can correct by calculation the measured value to eliminate the effects of the radiative diagrams. The effect of an emitted photon is to cause the calculated missing mass of the hadronic part to differ (be greater than) the actual missing mass. Thus data which should be binned at missing mass $M$ end up at missing 


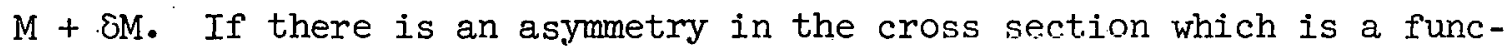
tion of missing mass, the radiative diagrams change that function to another one due to the redistribution of events in missing mass. In the simplest case, at the elastic peak in cross section (missing mass = $938 \mathrm{MeV})$, the cross section would be a delta function in missing mass without radiative diagrams and the region in missing mass between $938 \mathrm{MeV}$ and the inelastic threshold of $\mathrm{m}_{\mathrm{p}}+\mathrm{m}_{\pi}$ would have no events. With the radiative diagrams (acutal case, of course) the peak is smeared so that many events fall in this "empty" region and some even well beyond. The asymmetry in this "empty" region is the same as that at the elastic peak. In any place $M$ in the inelästic region there are both contributions from events of lower real missing mass and loss of asymmetry from events of real mass $M$ which are radiated off to higher missing mass. The radiative corrections were not made in our data. Since we are looking for a time-reversal violation and any asymmetry .. observed would be due to this, no matter what its missing mass (unless it is the small two- $\gamma$ exchange) and since there are no predictions as to what missing mass a $\mathbf{T}$ violation should occur at, why worry if we do not know the radiative corrections which might shift $20 \%$ of our data $50 \mathrm{MeV} 13,13$ when our resolution is not even that good?

It is important to note that we can only observe a $T$ violation in inelastic scattering. An asymmetry is precluded in elastic scattering by a combination of Hermiticity and gauge invariance ${ }^{14}$ in the one- $\gamma-$ exchange approximation regardless of $\mathrm{T}$ invariance.

The proofe we hovc given, while simple, du not result in an expression for a T-violating asymmetry in terms of the three form factors 
for the process. These are $F_{ \pm}$the two transverse (polarized virtual photon) and $F_{z}$ the longitudinal (polarized virtual photon) form factors. This required expression is given by Christ and Lee $^{9}$ in their notation in the lab frame:

$(\mathrm{w}, \underline{\mathrm{k}}),\left(\mathrm{w}^{\prime}, \underline{\mathrm{k}}^{\prime}\right)$ The 4 momentum of the initial and final electron

$\theta$

$\mathrm{M}_{\mathrm{T}}$

$q^{2}$

$\boldsymbol{v}$

$\underline{S}_{\text {in }}$

$\hat{n}$ Scattering angle of electron

4 momentum of final hadron state $\Gamma$

Effective mass of final hadron state $\Gamma$

$-(4 \text { momentum transfer })^{2}=\left(\underline{K}-\underline{K}^{\prime}\right)^{2}-\left(w-w^{\prime}\right)^{2}=4 w w^{\prime} \sin ^{2} \theta / 2$

The energy gained by the photon $=w^{-} w^{1}=q_{0}$.

Spin of target proton

Normal to scattering plane Texi

$$
\begin{aligned}
& \text { The double differental cross section is: } \\
& d \sigma=\frac{4 \pi \alpha^{2} w^{\prime}}{\left(q^{2}\right)^{2} w M_{\Gamma}} d w^{\prime} d(\cos \theta)\left\{g^{2} W_{1}+2\left(w w^{\prime}-g^{2} / 4\right) W_{2}+\operatorname{Sin}_{\text {in }} \cdot \frac{\left.\left(\underline{k} \times \underline{k}^{\prime}\right)\left(w^{2}-w^{\prime 2}\right) W_{3}\right\}^{\prime}}{m_{p}^{2}}(\text { II }-3)\right.
\end{aligned}
$$

where:

$$
\begin{aligned}
& W_{1}=\sum_{J} \sum_{\Gamma}\left\{\left|F_{+}\right|^{2}+|F|^{2}\right\} M_{\Gamma} \\
& W_{2}=\sum_{J} \sum_{\Gamma}\left\{\left|F_{+}\right|^{2}+|F|^{2}+g^{2}\left(E-m_{P}\right)^{-2}\left|F_{z}\right|^{2}\right\} m_{\Gamma} q^{2} / p^{2} \\
& W_{3}=2 i \sum_{T} 3 \sum_{\Gamma}\left\{I_{m}\left(F_{z}^{*} E\right)\right\} m_{\Gamma} m_{P}^{2} q^{2} p^{-2}\left(E-m_{P}\right)^{-2}
\end{aligned}
$$

where $\eta$ is a phase factor which depends on both the spin and parity of the final state $\mathrm{F}$.

$$
\eta=P_{\Gamma} \exp \left[i \pi\left(1 / 2-J_{\Gamma}\right)\right]
$$

The sum over $\Gamma$ is over all final spin orientations. To match our experimental. conditions we must also include tin the summation the sum over all outgoing total angular momenta $\mathrm{J}$ and all particle multiplicities at mass 
$M_{r}$.

The $\mathrm{W}_{3}$ term would produce a spin-dependent cross section (asymmetry). From Eq. (III-I) and (II-3) the asymmetry is.

$$
A=\frac{w w^{\prime} \sin \theta\left(w^{2}-w^{\prime 2}\right) W_{3}}{m_{p}^{2}\left[q^{2} w_{1}+2\left(w w^{\prime}-q^{2} / 4\right) W_{2}\right]}=\frac{\left(w^{2}-w^{\prime 2}\right) \cot \theta / 2 W_{3}}{m_{p}^{2}\left[2 W_{1}+w_{2} \cot ^{2} \theta / 2\right]}
$$

If time reversal invariance is correct then the F's are relatively real and thus $W_{3}=0$. For elastic scattering $F_{z}$ and $F_{-}$are linear functions of $G_{e}$ and $G_{m}$ and $F_{+}=0$ and, as is well known, $G_{e}$ and $G_{m}$ are real from Hermiticity. Hence there is no asymmetry in the elastic scattering case even if'there is a $T$ violation (in the one- $\gamma$-exchange approximation). As in all of Physics, there is at least one other notation ${ }^{15}$ in common use which will be stated here for future use. Let $\sigma_{t}$ and $\sigma_{L}$ be the cross sections for virtual transverse and longitudinal polarized photons on protons and $\sigma_{I}$ some cross section due to interference between the two. In terms of the form factors:

$$
\begin{aligned}
& \sigma_{t}=\frac{4 \pi^{2} \alpha}{K} \sum_{J} \sum_{\Gamma}\left\{\left|F_{t}\right|^{2}+\left|F_{1}\right|^{2}\right\}=\frac{4 \pi^{2}-}{M_{\Gamma} K} W_{1} \quad(I-\sigma) \\
& \sigma_{L}=\frac{4 \pi^{2} \alpha}{K} \frac{q^{2}}{V^{2}} \sum_{J} \sum_{\Gamma}\left|F_{z}\right|^{2}=\frac{4 \pi^{2} \alpha}{M_{\Gamma} K}\left[\left(1+2 / q^{2}\right) W_{2}-W_{1}\right] \\
& \sigma_{I}=\frac{4 \pi^{2} \alpha}{K} 2 \sum_{J} 3 \sum_{\Gamma} I_{m}\left(F_{z}^{*} E\right)=\frac{4 \pi^{2} \alpha}{M_{\Gamma} m_{p}^{2} K_{3}}\left(1+2 / q^{2}\right) \nu^{2} W_{3}
\end{aligned}
$$

where

$$
K=\frac{m_{\Gamma}^{2}-m_{P}^{2}}{2 m_{P}}
$$


$-20-$

is the energy of a real photon that would photoproduce $\Gamma$. To obtain the electroproduction cross section each of these must be multiplied by an appropriate factor $\Gamma_{i}$ (not to be confused with the final state $\Gamma$ ) representing the probability of the respective photon being emitted by the scattering electron.

$$
\frac{d \sigma}{d \Omega d \omega^{\prime}}=\Gamma_{t} \sigma_{t}+\Gamma_{L} \sigma_{L}+\Gamma_{I} \sigma_{I} \underline{S}_{\text {in }} \cdot \hat{n} \quad(I I-7)
$$

where:

$$
\Gamma_{t}=\frac{\alpha w \cdot R}{4 \pi^{2} w q^{2}}\left(2+\frac{\cot ^{2} \theta / 2}{\left(1+2 / q^{2}\right)}\right)
$$

$$
\Gamma_{L}=\frac{\alpha w^{\prime} K}{4 \pi^{2} w q^{2}}\left(1+2{ }^{2} / q^{2}\right)^{-1} \cot ^{2} \theta / 2
$$

$$
\Gamma_{I}=\frac{\alpha w^{\prime} K}{4 \pi^{2} w g^{2}} \frac{w+w^{\prime}}{\left(1+2^{2} / q^{2}\right) 2} \cot \theta / 2
$$




\section{Analysis of Kinematic Factors}

There is no theory predicting the kinematical conditions in which one might expect a T-violation to appear in its largest form if it appears at all. There is a conjecture made by both Lee ${ }^{16}$ and okun ${ }^{14}$ that the $T$ violation in electromagnetic interactions should obey a $\Delta T=0$ rule. Lee's proof assumes that the CP-violating current transforms like its charge under Isospin rotations (minimal electromagnetic interactions). Okun has a more general proof which he seems to have restricted. ${ }^{18}$ If one believes this rule then one would not expect to find an asymmetry at the $\triangle_{33}(1236)$ resonance which is a $\Delta I=1$ transition from the $I=1 / 2$ proton.

Even if one assumes a maximal $\mathrm{T}$ violation everywhere, there is still great uncertainty as to the best place to look for it. Since the cross-section dependence on polarization of the proton target is given by Eq. (I王 +7$)$ we can write the Asymmety parameter

$$
A=\frac{\sigma_{1}-\sigma_{t}}{\sigma_{t}+\sigma_{t}}
$$

a.s

$$
A=\frac{\Gamma_{I} \sigma_{I}}{\Gamma_{t} \sigma_{t}+\Gamma_{L} \sigma_{L}}
$$

Expanding, we get:

$$
|A|=\frac{2 q_{\%} / q \Gamma_{I} \sin \phi \sqrt{\sigma_{L} / \sigma_{t}}}{\Gamma_{t}+\Gamma_{L} \sigma_{L} / \sigma_{t}} \frac{|E|}{\left[\left|F_{t}\right|^{2}+|E|^{2}\right]^{1 / 2}}
$$

where only one term in the sum (Eq. (II-6)) has been taken and $\varphi$ is the phase between $F_{-}$and $F_{z}$ which may be $90^{\circ}$ for maximal $T$ violation. our ignorance is at once apparent when you realize that $\sigma_{I} / \sigma_{t}\left(q^{2}, M_{\Gamma}\right)$ is 
known ${ }^{19}$ for only a few values of $q^{2}$ and $M_{\Gamma}$ and not known very well even there.

$$
\frac{|E|}{\left[\left|F_{+}\right|^{2}+|E|^{2}\right]^{1 / 2}}
$$

cannot be determined from cross-section measurements (as seen from (II-3) and $(I I-4))$. Il ls llus unly inferied from theoretical predictions ${ }^{11}$ of the production threshold behavior of the amplitudes.which depends in turn upon which multipole produces the resonances. In the kinematic region experimentally accessible to us

$$
\Gamma_{t} \approx \Gamma_{L} \approx q_{0} / q \Gamma_{I}
$$

which simplifies our expression to

$$
|A|=R \sin \theta|F| /\left[\left|F_{+}\right|^{2}+|E|^{2}\right]^{1 / 2}
$$

where

$$
R=\frac{2 \sqrt{\sigma_{1} / \sigma_{t}}}{1+\sigma_{t} / \sigma_{t}}
$$

A plot of $R$ vs $\sigma_{I} / \sigma_{t}$ (Fig. 3) indicates, fortunately, that $R$ is not very sensitive to $\sigma_{I} / \sigma_{t}$. With $R=R_{\max }=1$ and

$$
|F| /\left[\left|F_{+}\right|^{2}+|E|^{2}\right]^{1 / 2}
$$

maximum value $=1$, the asymmetry $|A|=I$ which is the maximum it can obtain. Thus on the basis of our negligible knowledge of the form factors it is not possible to determine optimum kinematic conditions for observation of a possible $T$ violation. 


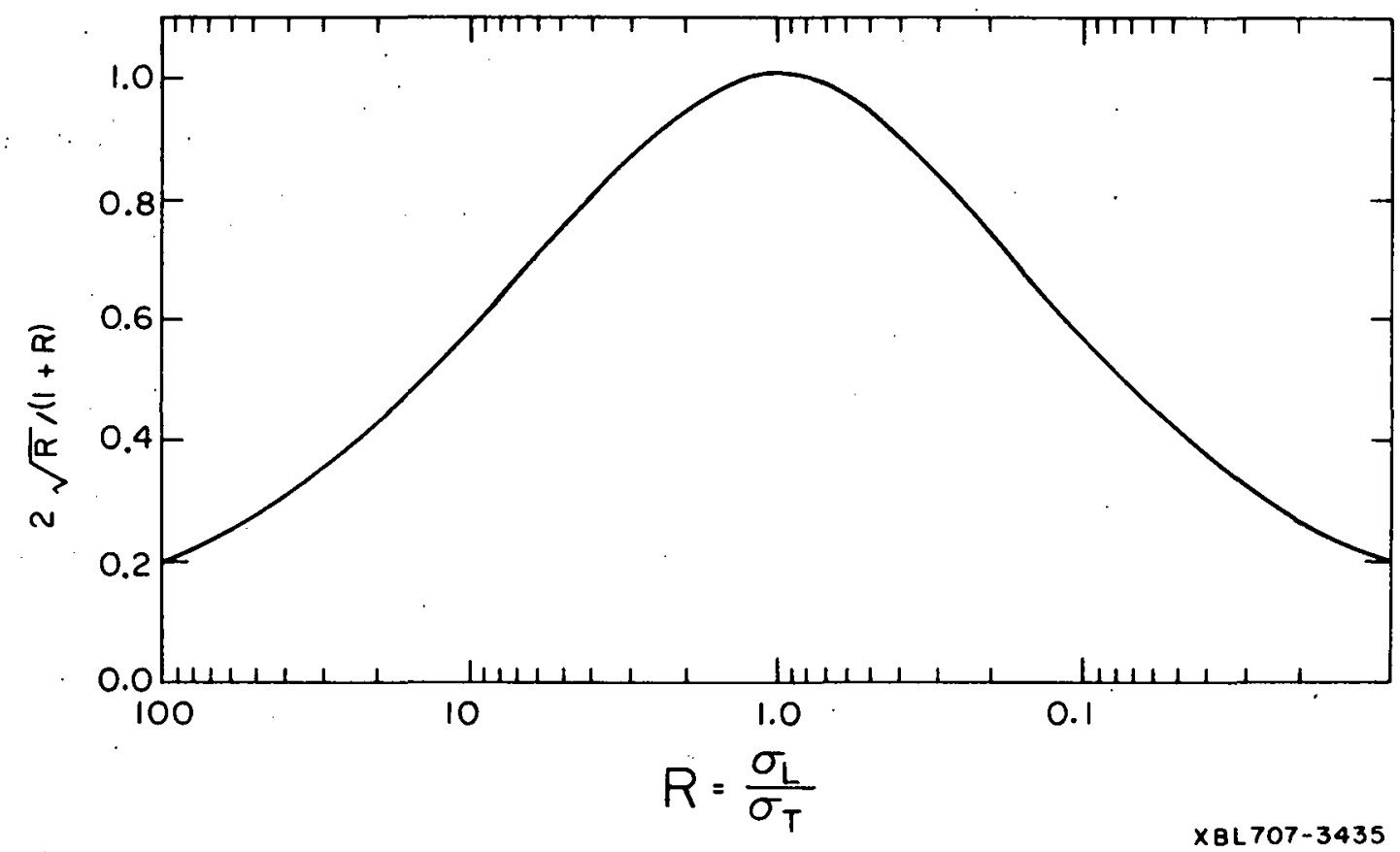

Fig. 3. The function $2 \sqrt{R} /(1+R) \cdot R=\sigma_{I} / \sigma_{t}$ 
A more reasonable, but still crude, estimate of $A$ can be obtained at the $\Delta_{33}(1236)$ resonance. It dominates the cross section, is known to be mostly a magnetic dipole transition $11,20 \mathrm{So}^{21}$

$$
\left|F_{-}\right| /\left[\left|F_{+}\right|^{2}+\left|F_{-}\right|^{2}\right]^{1 / 2}=1 / 2
$$

and has $\sigma_{I} / \sigma_{t}=0.14 \pm 0.07$ at $q^{2}=0.6$. Thus $|A| \approx 0.03 \sin \theta$. A similar estimate at other mass values is even more uncertain due to the contribution of many other values of anguiar momentum and particle multiplicities (as indicated in the sum in Eq. (II-4)) instead of a dominant resonance. Hence one can expect if BFL is right, a rather large polarization but not with overwhelming confidence. This issue is discussed further in the interpretation of the data, section IX. 
D. Running Conditions.

Without theoretical guidance, we were free to operate the experiment at kinematic conditions dictated largely by experimental feasibility. Table I gives the values of $\mathrm{q}^{2}$ and $\mathrm{M}_{\Gamma}$ at which we took data. The procedure used was:

(1) Select beam intensity so as to damage the target in a "reasonable" time (a few hours, see section $V$ on target);

(2). Select energy of beam for highest cross section (highest energy);

(3) Select scattering angle so that the counting rate is as large as we can handle comfortably (see section IV on counting rate).

Note that, the cross section depends sharply on scattering angle, as $1 / \theta^{8}$, but is not very dependent on the scattering momentum; or, in other. symbols, $\sigma \alpha \mathrm{q}^{-4}$ and $\sigma$ independent of missing mass. This latter fact is the surprising result of SLAC experiments. ${ }^{13}$ These criteria resulted in most of our running being at $w=18 \mathrm{GeV}$ and $q^{2} \cong 0.6\left(\theta=2.51^{\circ}\right)$, with less detailed exploration at "high" $\mathrm{q}^{2}\left(\approx \mathrm{I}(\mathrm{GeV} / \mathrm{c})^{2}\right)$ and How" $\mathrm{q}^{2}\left(\stackrel{\sim}{:} \cdot 0.4(\mathrm{GeV} / \mathrm{c})^{2}\right)$. 
$-26-$

TABLE I. RUNNING CONDITIONS

\begin{tabular}{|c|c|c|c|c|c|}
\hline $\begin{array}{c}\text { Beam } \\
\text { Particle } \\
\end{array}$ & $\begin{array}{c}\text { Beam } \\
\text { Energy } \\
\text { GeV } \\
\end{array}$ & $\begin{array}{l}\text { Scattering } \\
\text { Angle } \\
\text { (deg) } \\
\end{array}$ & $\begin{array}{c}\text { Missing Mass } \\
\text { Range } \\
\text { GeV } \\
\end{array}$ & $\begin{array}{c}\text { 4-momentum } \\
\text { Transfer Squared } \\
(\mathrm{GeV} / \mathrm{c})^{2} \\
\end{array}$ & $\begin{array}{l}\text { Number } \\
\text { of Events } \\
\left(\mathrm{x} 10^{6}\right) \\
\end{array}$ \\
\hline$e^{-}$ & 18 & 2.48 & $\begin{array}{c}\text { elastic-1.910 } \\
2.350-2.650\end{array}$ & .58 & 64 \\
\hline$e^{-}$ & 18 & 3.21 & elastic-2.150 & .96 & 10 \\
\hline$e^{-}$ & 15 & 2.37 & elastic- 1.850 & .37 & 15 \\
\hline$e^{+}$ & 12 & 3.18 & $1.050-1.450$ & .42 & 14 \\
\hline
\end{tabular}




\section{EXXPERIMENTAL DESIGN}

\section{A. Asymetry Formula}

It is necessary to find the Asymmetry A from the experimental situation in which the target is not all free protons and those protons can only be partially polarized. Our target consists of $n_{\uparrow}\left(n_{\downarrow}\right)$ free protons with spins parallel (antiparallel) to the scattering plane and $\mathrm{n}^{\mathrm{c}}$ other nucleons (bound in various nuclei). The cross section for the entire target is

$$
\sigma=\left(n_{\uparrow} \sigma_{\uparrow}+n_{\downarrow} \sigma_{\downarrow}+n \sigma_{c}\right) /\left(n_{\uparrow}+n_{\downarrow}+n^{c}\right)
$$

where the $\sigma^{\prime}$ s are the cross sections of the particular nucleons indicated. The unpolarized cross section (when the average free: proton spin is 0 ) is

$$
\sigma_{0}=\frac{\frac{n}{2}\left(\sigma_{\uparrow}+\sigma_{\downarrow}\right)+n^{c} \sigma_{c}}{n_{\uparrow}+n_{\downarrow}+n^{c}}
$$

where $n=n_{\downarrow}+n_{\uparrow}$. is the total number of free protons. Then:

$$
\begin{aligned}
\sigma & =\sigma_{0}+\sigma_{+}\left(\frac{n_{+}-n_{t}}{2}\right)-\sigma_{t}\left(\frac{n_{+}-n_{t}}{2}\right) \\
\therefore & =\sigma_{0}\left[1+\frac{\sigma_{+}-\sigma_{t}}{\sigma_{+}+\sigma_{t}} \cdot \frac{n_{1}-n_{t}}{n_{+}+n_{t}} \cdot \frac{n / 2\left(\sigma_{+}+\sigma_{t}\right.}{\sigma_{0}}\right]=\sigma_{0}\left[1+A \cdot P \cdot H_{f}\right]
\end{aligned}
$$

where $A$ is defined in Eq. (II-I), $P=\frac{n_{1}-n_{t}}{n_{q}+n_{t}}$ is the polarization of the free protons in the target and $H_{f}$ is the fraction of the cross section which comes from the free protons (hydrogen). The experimental procedure is to evaluate $\sigma$ for opposite values of $\mathrm{P}= \pm \mathrm{p}$, yielding $\sigma_{ \pm}=\sigma_{0}\left(1 \pm \mathrm{ApH}_{f}\right)$. Hence: 


$$
\varepsilon=\frac{\sigma_{+}-\sigma_{-}}{\sigma_{+}+\sigma_{-}}=A p H_{f}
$$

Thus: $\quad A=\frac{1}{P H_{f}} \varepsilon$

where $\epsilon$ is the experimentally measured asymmetry in the counting rate.

Since we measure only the ratio of cross sections it is unnecessary to obtain absolute cross sections. This means it is unnecessary to know the efficiency of the detection system and the normalization of the beam monitors. However, whatever they are, they must remain constant.

The uncertainty in $\mathrm{A}$ is simply:

$$
\frac{\Delta A}{A}=\sqrt{\left(\frac{\Delta \varepsilon}{\varepsilon}\right)^{2}+\left(\frac{\Delta P}{P}\right)^{2}+\left(\frac{\Delta H_{f}}{H_{f}}\right)^{2}}
$$

When $\epsilon$ is near zero (our case) and the other errors are relatively small one gets:

$$
\begin{aligned}
& \left(\frac{\Delta \varepsilon}{\varepsilon}\right)^{2} \gg\left(\frac{\Delta P}{P}\right)^{2} \\
& \left(\frac{\Delta \varepsilon}{\varepsilon}\right)^{2} \gg\left(\frac{\Delta H_{f}}{H_{f}}\right)^{2}
\end{aligned}
$$

and Thus:

$$
\Delta A=\frac{\Delta \varepsilon}{P H_{f}}
$$$$
\text { (III -3) }
$$

It is clear from this expression that the uncertainty in the knowledge of $\mathrm{P}$ and $\mathrm{H}_{\mathrm{f}}$ would only multiply $\mathrm{A}$ and the error in $\mathrm{A}$ by an unknown constant and not effect what we are really interested in: is $A \neq 0$ to any statistical significance (see section VII for details). It is the uncertainty in $\epsilon$, i.e. anything which might cause an apparent discrepancy between $\sigma_{+}$and $\sigma_{-}$(aside from actual $\mathrm{T}$ noninvariance or 
$-29-$

two- $y$ exchange), which is a threat to the success of the experiment. In order to evaluate $A$ to a few percent with typical values of $H_{f}=0.1$ and $P=0.2$ we find that $\epsilon$ must be known to a few hundredths of a percent. For this statistical accuracy we need something of the order of ten million events and, what is potentially more bothersome, we must prevent (or at the very least know about) nonstatistical errors of this size. 
B. Errors and Target Polarization Reversals

We expect this experiment to be relatively free of any systematic error since our apparatus remains almost literally unchanged, unmoved, and untouched for measurements of both $\sigma_{+}$and $\sigma_{-}$. The only change is a shift of $0.3 \%$ in microwave frequency to alter the target polarization. Since the microwave power does not effect the operations in any way except changing the target polarization (for possible complications see Section V.D), there is an inherent lack of sources of systematic error in this experiment. Nevertheless at our desired level of accuracy the greatest vigilance is necessing if instrumental fluctuations are not to effect the results.

Our procedure of taking data alternately with opposite signs of target polarization divides possible nonstatistical errors into two categories:

(1) Those distumbing phenomenon which are random i.e. uncorrelated with target polarization, and

(2) Those which are correlated.

Of course the best techniques to rid oneself of both classes of error is perfect equipment and eternal vigilance, but, both being in short supply, type-1: errois can be reduced in effect by another technique. By reversing the target polarization frequently, we can arrange that a random disturbance, such as f'luctuating detection ef't'iciency or position jitter of the beam, will tend to contribute equally to both $\sigma_{+}$and $\sigma_{-}$and hence cancel out in the asymmetry calculation. The types of fluctuation with which we have to deal determines how rapidly they will cancel out due to polarization reversal. If the random disturbances are slow or have 
a lower frequency then the polarization reversal frequency (as, for example, a slow drift in counter efficiency) the:error induced will decrease inversely as the reversal frequency for a fixed total interval of data taking. This is also true if the random disturbance is a step function (such as might be due to the beam shifting position suddenly) or to any number of step functions located randomly in time. However, if the disturbances are delta functions (very short in duration) or any random combination of delta functions (such as a power surge changing efficiencies for a very short time) or if the fluctuations are very rapid with respect to the polarization frequency (such as beam position. jitter due to jitter in magnet power supplies), then the error induced is independent of the polarization reversal frequency for a fixed total interval of data taking. For all cases, the induced error will be reduced when one takes data for a longer interval of time $T$ by a further factor proportional. to $I \sqrt{T}$. Further discussion and proofs of the above statements are in Appendix A.

The contributions of the fluctuations are thus reduced significantly by increasing the polarization reversal frequency and this was our major defense against random errors. For example, inconsistancies as high as $1 \%$ were tolerated in the monitors over the time between polarization reversals but these were found to be reduced to $\leq 0.01 \%$ after a few thousand reversals.

In our finite period of running all random fluctuations will not necessarily cancel out. A frequency spectrum of this noise would probably show contributions near our polarization reversal frequency which could induce a small error in the asymmetry. In the Analysis section 
we describe how this possible noise is measured and show that these errors are small compared to normal statistical counting errors.

The error not reduced by long running and frequent polarization reversal is that which is due to a physical bias in our apparatus. Possible effects include the potential of a correlation of target density with target polarization (varying liquid helium level, discussed in Target Section) and the problem of a non-uniform target thickness combined with changing beam position. Most of the rest of this paper is devoted to describing the techniques used to avoid physical bias in our apparatus. 


$$
-33-
$$

\section{Summary}

The most important experimental features of this test of $T$ invariance are designed to overcome the problems mentioned above. These are:

(I) A polarized proton target which can reverse polarization rapidly and is not too much damaged by high beam intensities,

(2) A system of sweeping the beam back and forth over the entire target to uniformly irradiate it,

(3) Computer control over everything to insure that each counting interval was handled in a standard manner. 
IV. - EXPERTIMENTAL SETUP

\section{A. Beam}

The experimental apparatus are shown in Fig. 4. Electrons (or positrons) from the SLAC $20-\mathrm{GeV}$ accelerator were momentum analyzed to a total $\Delta p / p$ of $0.2 \%(0.3 \%)$. This beam was swept left-right and up-down by a pair of air-core Helmholtz coils under Computer control to uniformIy distribute radiation damage to the target. After each beam pulse (1.6 $\mathrm{\mu sec}$ long, $5 \mathrm{~ms}$ apart) the magnet current was stepped to move the beam $1 / 12$ of an inch at the target. A complete pattern, shown in Fig. 5 covered the entire target and included 288 spots taken in a systematic down sweep of 12 horizontal lines each with 12 points followed by an upsweep of another interlaced 12 horizontal lines. A complete pattern, covering the entire target, took about $1-1 / 2$ seconds at 180 pps (pulses per second).

The beam intensity was measured by two induction toroids 22 placed upstream of the target and a secondary emission quantometer 23 which also acted as a beam dump 200' behing the target. Absolute calibration of the monitors was unnecessary, but we could not tolerate fluctuations in sensitivity as this might lead to erronious asymmetries. During running the three monitors were required to agree with one another to better then $4 \%$ for each subrun of data taking ( 6 complete beam sweep patterns, about 10 seconds) in order for the data to be included in the analysis.

The position of the beam in space was monitored in an $R F$ cavity $^{24}$ placed upstream of the sweeping magnets. This position reading was displayed continuously on an oscilloscope and was helpful in detecting 
(Not to scale)

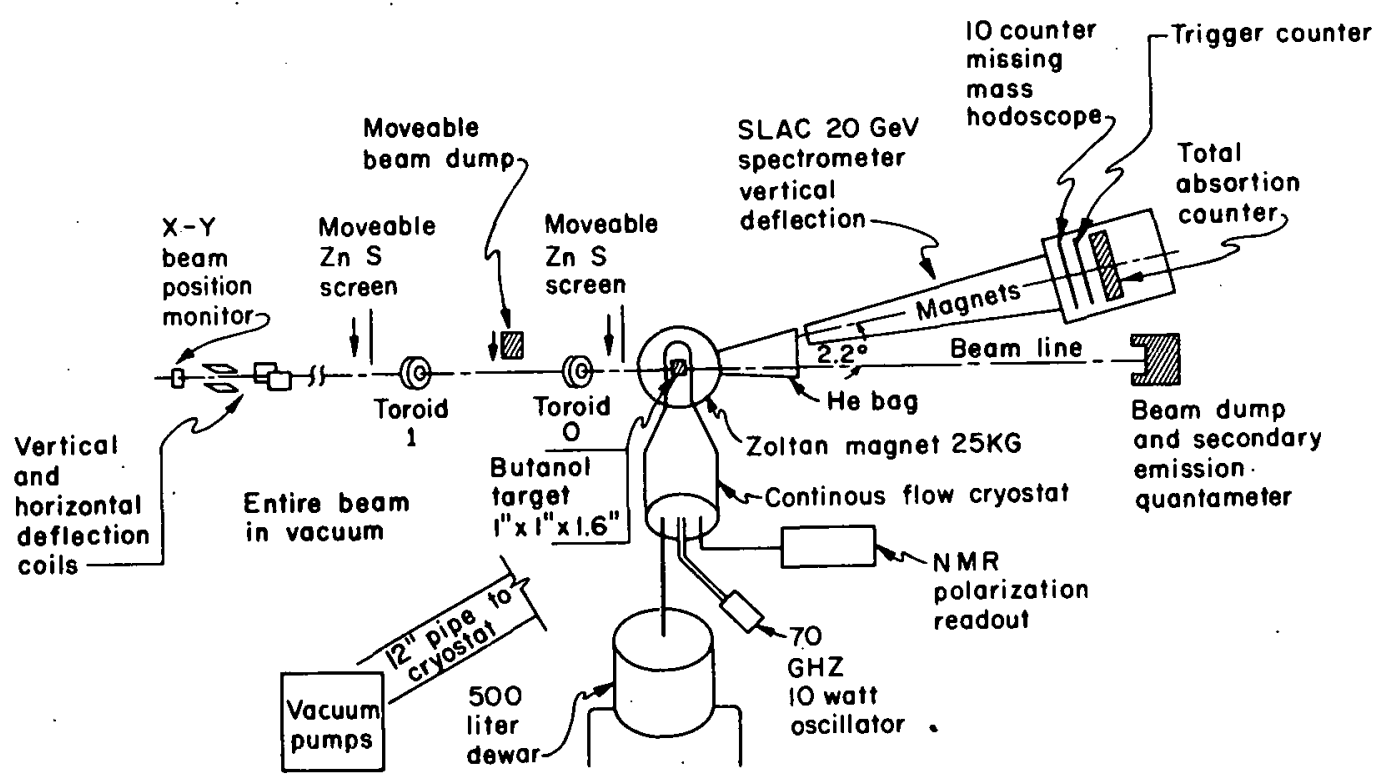

XBL69II-6279

Fig. 4. Schematic diagram of experimental apparatus. 


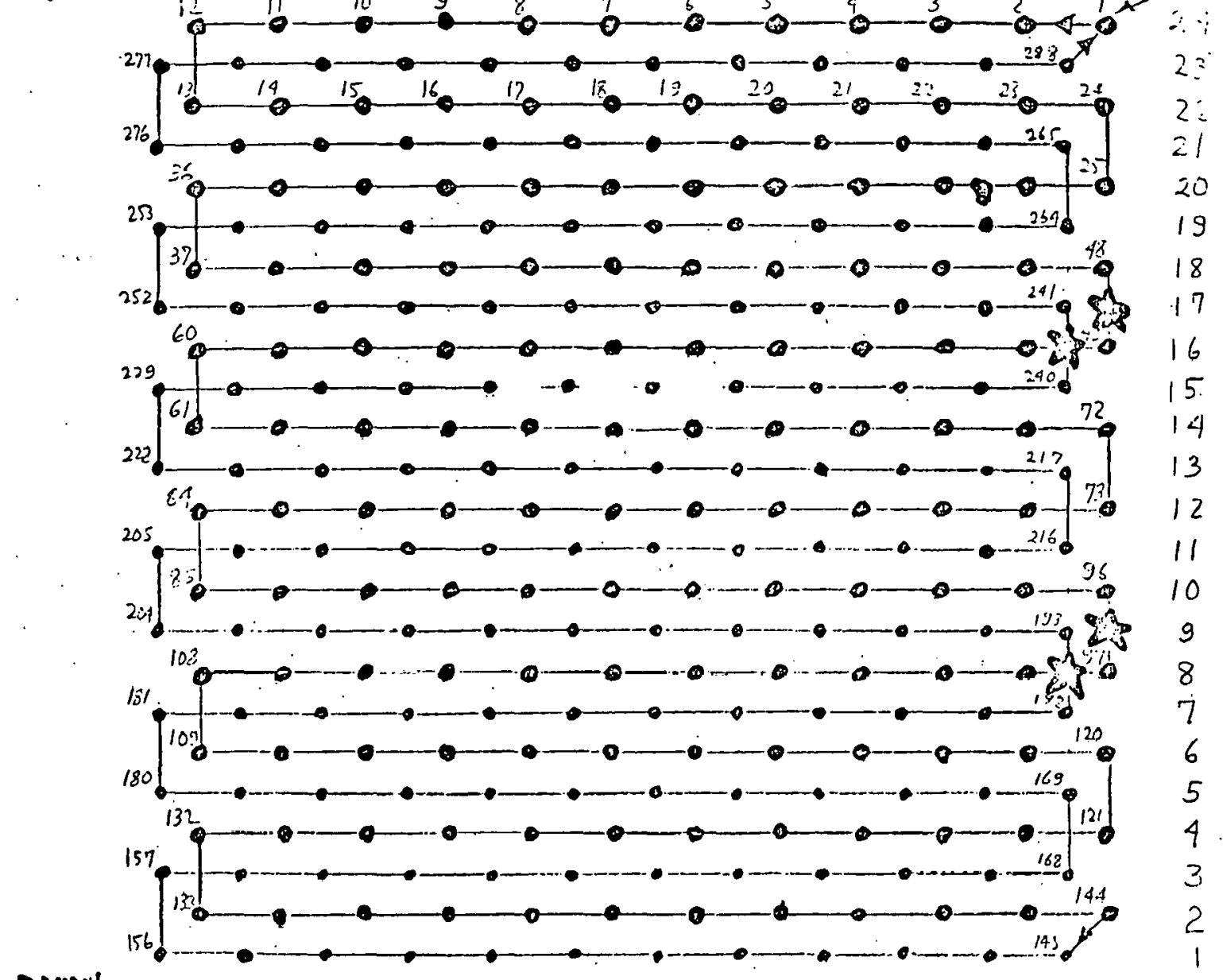

\section{BEAMPS LEFT}

BEARS RIBITT

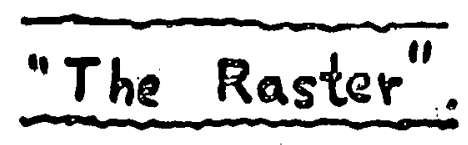

XBL 708-1713

Fig. 5. The Raster. The pattern of beam position at the target due to the sweeping magnets. The dots indicate the position at beam pulses. The upper right is the starting position. The stars indicate the boundaries bewtween the regions on the upsweep and downsweep. 
changes of beam conditions. The distribution of the beam in time was recorded by a Cherenkov counter placed near the target and was displayed on another oscilioscope as a further check on beam conditions.

The beam, when it reached the polarized target, was about $2-3 \mathrm{~mm}$ in diameter as observed by the darkening of glass slides placed at the target position. When the beam was swept by the magnets it uniformly irradiated (to within about $10 \%$ ) an area 1 " $x .1$ " corresponding to the size of the target, again as observed by a glass slide placed in the beam. The beam could be observed on two thin, removable ZnS screens placed upstream of the target and monitored by remote TV. To avoid radiation damaging: the target while the beam was being tuned, we arranged that a 30-radiation-length metal block could be remotely moved into the beam in front of the target. 


\section{B. Detection Equipment}

Scattered electrons from the polarized proton target were detected in the SLAC $20-\mathrm{GeV}$ spectrometer. 25 The small solid angle of acceptance of $10^{-4} \mathrm{sr}$. and momentum acceptance of $\pm 2 \%$ limited our counting rate. A ten-counter hodoscope was oriented so that each counter subtended a combination of angle $(\theta)$ and momentum $\left(w^{\prime}\right)$ corresponding kinematically to a fixed missing Mass $\mathrm{M}_{\mathrm{T}}$ of the undetected particles. Since:

$$
M_{\Gamma}^{2}=m_{p}^{2}-4 w w^{\prime} \sin ^{2}(\theta / 2)+2\left(w-w^{\prime}\right) m_{p}
$$

the condition for constant $\mathrm{M}_{\mathrm{T}}$ is:

$$
d M_{r}^{2}=0=-2 W W^{\prime} \theta d \theta-2 m_{p} d W^{\prime}
$$

Thus:

$$
\frac{d w^{\prime}}{d \theta}=-\frac{w w^{\prime} \theta}{m_{p}}
$$

The $20-\mathrm{GeV}^{\prime}$ spectrometer has an angle measuring focal plane perpendicular to the optical axis of the spectrometer with a first-order optical coefficient $d \theta / d x=6.5 \times 10^{-4}$ where the scattering angle $\theta$ is in radians an the horizontal displacement at the focal plane $x$ is in $\mathrm{cm}$. The momentum measuring focal plane is tilted at an angle of $43^{\circ}$ with respect to the optical axis about a horizontal line. The corresponding firstorder optical coefficient is $\frac{d y}{d w^{t} / w^{\top}}=283$ where $y$ is the displacement in $\mathrm{cm}$. along the momentum focus in the near vertical direction. The orientation of the missing mass hodoscope, dy/dx, is thus fixed by:

$$
\left.\frac{d y}{d x}\right|_{M_{\Gamma}}=\left.\left.\left.\frac{d y}{d w^{\prime}}\right|_{\theta} \frac{d \theta}{d x}\right|_{w^{\prime}} \frac{d w^{\prime}}{d \theta}\right|_{M_{r}}=\frac{-18 w \theta}{m_{p}} \approx .15
$$


at our typical running conditions of $w=18 \mathrm{GeV}$ and $\theta=2.5^{\circ}$. A slope of 0.15 mean that the missing mass as seen in the spectrometer is much more sensitive to momentum plane position than angle plane. Hence our hodoscope was oriented in a manner similar to the momentum plane hodoscope except for a tilt of about $9^{\circ}$ (depending on kinematics) around the optical axis of the spectrometer. Each of the $0.45^{\prime \prime} \times 0.45^{\prime \prime} \times 7 "$ counters subtended $10 \%$ of the total $\Delta p / p$ acceptance of $4 \%$ of the spectrometer.

To differentiate electrons from other particles which might be present a 16-radiation-length lead-scintillator sandwich Total-Absorption counter (TA) was placed behind the hodoscope. Electrons, because of their low mass, will create showers, and a large amount of light, while $\mu$ 's, $\pi$ 's and other heavy particles will not make showers, and will leave only a minimum ionization energy behind. The output from the TA counter was pulse-height discriminated to pick only those particles creating large amounts of light. The separation from minimally ionizing particles was quite clean, as is indicated in Fig. 6. Using the information from two $\mathrm{dE} / \mathrm{dx}$ counters which were placed behind steel sheets, and thus measured the initial stages of a shower, we were able to study a sample of those events which might be ambiguous in the TA counter alone. Using this additional knowledge we est1mate the non-electron contamination in our final Asymmetry A to be less than $0.2 \%$ and thus of no significance even if there were a 100\% asymmetry associated with them. A trigger counter was placed right behind the hodoscope. A fast triple coincidence between the TA counter, lrigger counter and one of the missing-mass-hodoscope 
$-40-$

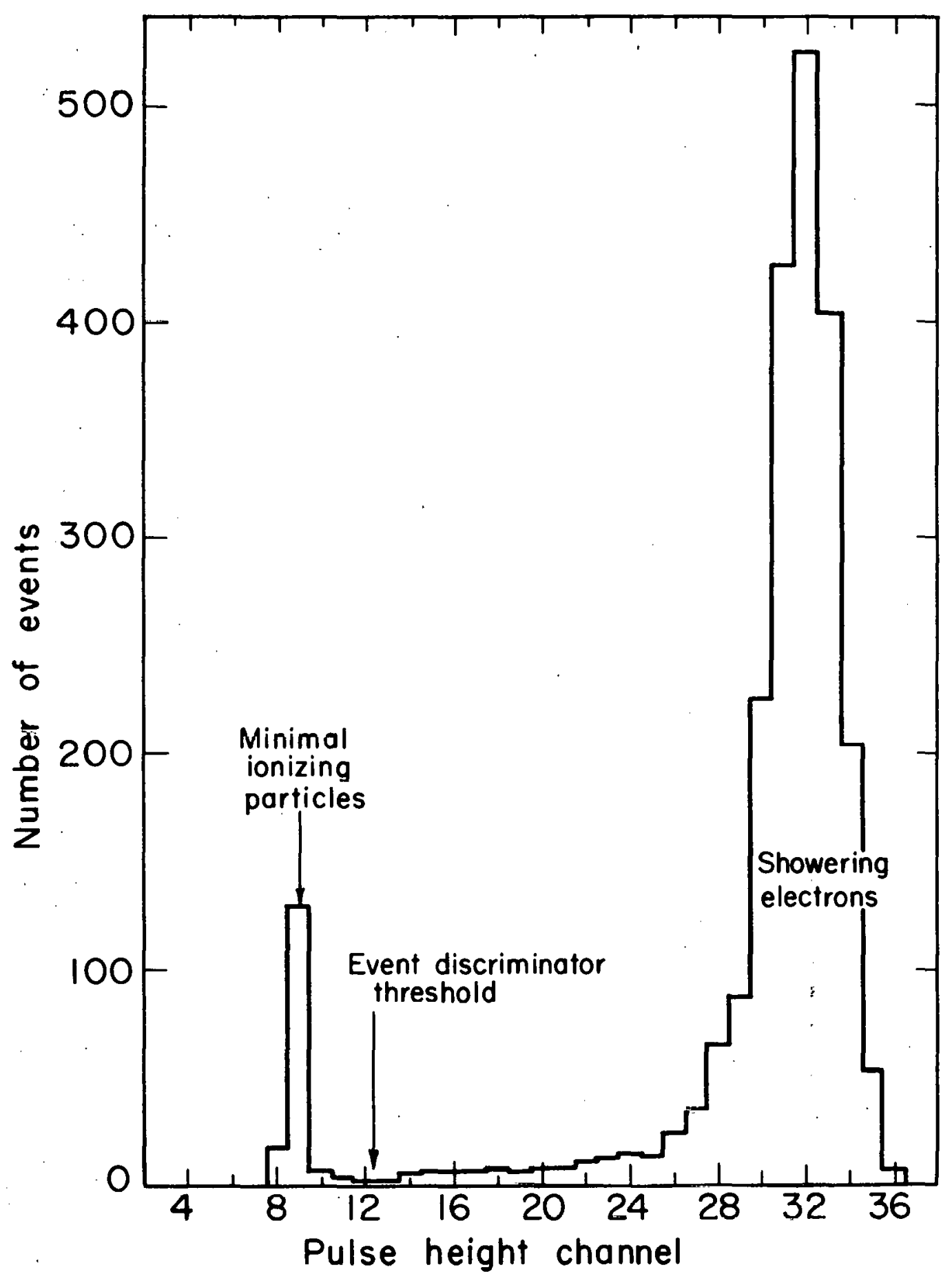

XBL706-3203

Fig. 6. Total-absorption lead-scintillator shower counter spectrum. Only events in which the pulse height was greater than channel 12 were accepted. Pion contamlnation of these events is about $0.2 \%$. 
$-41-$

counters defined an event and advanced one of ten $100 \mathrm{Mc}$ scalers. Count was also kept of the triggers (coincidences between TA and trigger counter), accidental or change coincidences (coincidence between TA and trigger counter delayed by $20 \mathrm{~ns}$ ) and singles in both TA and trigger counters. The electronics wiring is shown in Fig. 7 .

The time resolution of the system was about $7 \mathrm{~ns}$, as indicated by delay curves and accidental rates (accidental rate) $=(\text { trigger rate })^{2}$ $\mathrm{x}$ (resolution time), where typical running conditions were accidental rate $=1.1 \%$ and 1.5 triggers per $1.5 \mu \mathrm{sec}$ pulse) .

The optics of the spectrometer are quite sensitive to the vertical. position of the interaction point. The vertical magnification of the spectrometer is about 0.9 . Thus corrections must be made for the beam position sweep of $\pm 1.3 \mathrm{~cm}$ described above. Since each missing mass counter is $1.15 \mathrm{~cm}$ and this is the ultimate limitation on our $\Delta p / p$ resolution, we could simply divide the data from the target into three vertical regions (called top, middle, and bottom) of $0.85 \mathrm{~cm}$, each of which, when projected onto the hodoscope plane, subtends about $0.8 \mathrm{~cm}$. This data division was accomplished by having the computer read the contents of the ten missing-mass scalers and one of the monitors every time the beam crossed from one region to another (about 3 times per second) and keep separate totals for each oflthe regions. After the appropriate kinematic corrections of shifting top and bottom regions one bin (in opposite directions of course) 12 missing-mass bins were created from the 10 counters when the data was combined.

The presence of the polarized-target magnet at the scattering center also necessitated kinematic corrections to the normal spectrometer 
$-42-$

\section{Accidentals Triggers}

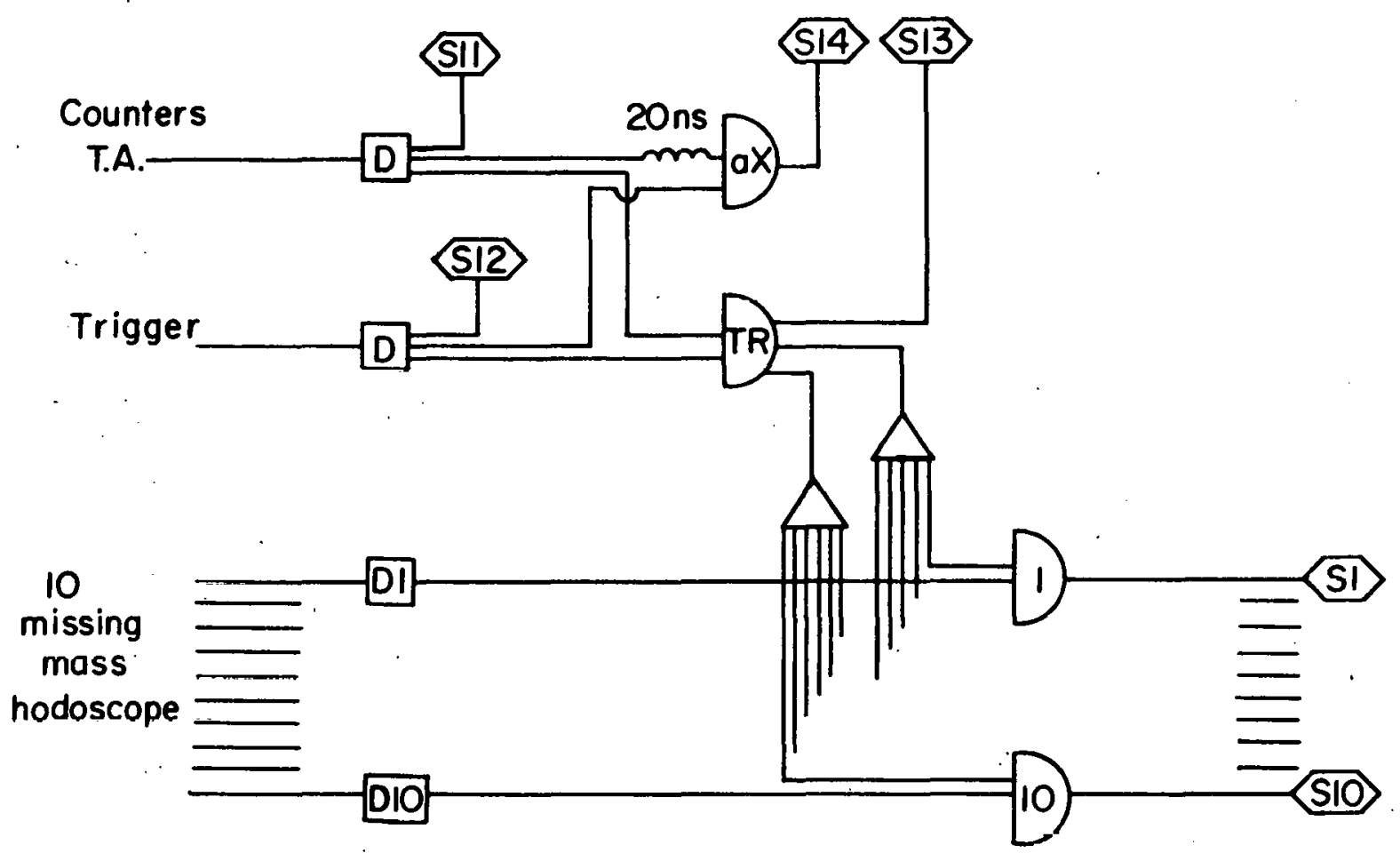

XRI. 7กตุ-32ก4

Fig. 7. Electronic Logic diagram of the event processing circuitry. D. stands for pulse-height discriminator, $S$ for scaler and $\Rightarrow$ for "AND" coincidence circuit. The discriminators and coincidences are $200 \mathrm{MHz}$ and the scalers $100 \mathrm{MHz}$. 
optics. The bending of the magnet, proportional to $\int \mathrm{Bdl}$, was measured by integrating the charge from a wire coil moved through the magnet and, independently, by observing the bending of the unscattered electron beam with a lever arm of $200^{\prime}$. The methods agreed to better than $1 \%$ and gave about $1^{\circ}$ (depending on beam energy). For futher details on this and other kinematic corrections see Appendix B.

The "hut" at the back end of the spectrometer had been equipped with several complicated arrays of hodoscopes for fine resolution of momentum, production angle, azimuthal angle, position of the interaction within the target and particle identification. This was referred to as the "old" or "slow" system. 26 since a slow event required reading out many banks of hodoscopes from flip-flops, only one could be handled by the computer per beam pulse; to avoid large deadtime problems only a small fraction of an event/pulse could be accepted. Instead of removing the slow system, we recorded every fiftieth "slow" event on magnetic tape for future use. (The computer did not have time to handle more.) This data was then used to determine our resolution (the "old" resolution was well known and four times better then ours), the non-electron contamination discussed above and to give us a sense of security. 


\section{Resolution}

Ideally the resolution in missing mass of our detection system would be determined by the $0.4 \%$ momentum bite of each missing-mass counter (since, as shown above, the production angle is unimportant). Unfortunately the following distortions reduced this resolution:

(1) The missing-mass hodoscope was placed upstream of the momentum focus so that it would not be behind another "old" array of counters. When the missing-mass hodoscope had been temporarily located behind the angle and momentum hodoscopes, $\delta$ rays created in these hodoscope scintillators had distorted the data from the missing-mass hodoscope.

(2) The sweeping of the beam across the target changed the kinematics of the spectrometer. Fiven with the divisinn of the target into three kinematic regions this hurts the resolution.

(3) There are still $\delta$ rays which cause $\leq 10 \%$ of our events to trigger two or more counters (usually two adjacent counters).

We compared some of the data event by event in the missing-mass hodoscope with that in the "old" system whose more accurate resolution is well known. This comparison, illustrated in Fig. 8, shows that our resolution $(F W H M)=0.6 \% \Delta p / p$ or about $1-1 / 2$ counter-width bins: This corresponds to a missing-mass resolution varying between $100 \mathrm{MeV} F \mathrm{FHM}$ at the elastic peak to $60 \mathrm{MeV}$ FWHM at the $\mathrm{N}^{*}(1688)$. This limits our ability to see a rapidiy varying asymmetry which crosses zero frequently. It was a sacrifice we made to obtain a large counting rate and good statistics by using the missing-mass hodoscope. 


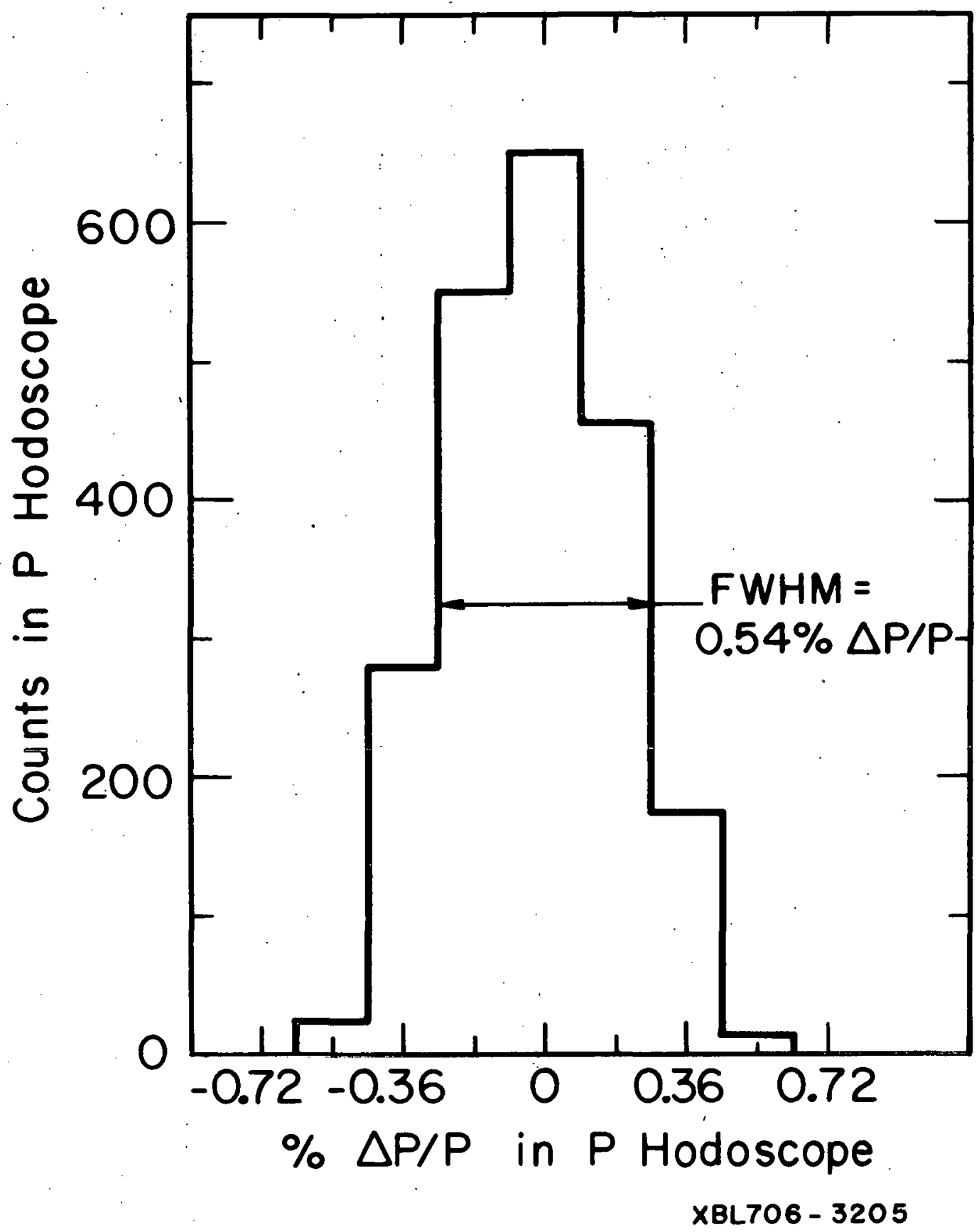

Fig. 8. Resolution of the detection system. Distribution of events from one missing mass hodoscope counter in the momentum hodoscope. (Beam sweep off.) 
$-46-$

\section{POLARIZED PROTON TARGET}

\section{A. Principles of Operation}

The polarization of free protons in a magnetic field $H$ and at a temperature $T$ is given by the Boltzman distribution

$$
\begin{aligned}
& P=\frac{1-\exp \left(-\mu_{N} g_{P} H / k T\right)}{1+\exp \left(-\mu_{N} g_{P} H / k T\right)} \\
& P=\tanh \left(\frac{\mu_{N} g_{P} H}{2 k T}\right)
\end{aligned}
$$

For temperatures obtainable in the large thin-walled apparatus capable of absorbing, milliwatts of energy, necessary for high-energy physics experiments, $\left(0.5^{\circ} \mathrm{K}\right)$ and for magnetic fields available $(50 \mathrm{kG})$ the polarization amounts to only a few percent and is thus useless for scattering experiments. Even if lower temperatures or higher fields . could be achieved, hydrogen, the most desirable substance to use because it is all free protons could not be used because it occurs as molecules, the low energy state of which is nuclear-spin-zero and therefore unpolarizable.

The technique of Dynamic Nuclear Orientation seeks to overcome these difficulties by. microwave pumping the protons into one of their spin states and depleting the other. Only cerain exotic mixtures containing paramagnetic substances have the distribution of magnetic energy levels necessary for the technique. This method has the inherent advantage that the polarization can be rapidly reversed without changing any part of the experimental apparatus except a small change in the microwave pumping frequency. The brute force method described above would require a change in the direction of the magnetic field, with all the 
introduction of systematic errors associated with moving the rest of the detection apparatus to compensate.

The principles of Dynamic Nuclear Orientation have been described by many people 27 more competent than I to extol its theoretical mechanisms. The central idea behind the process is that unpaired electrons or electron-like magnetic moments are introduced into a target material creating in a large magnetic field energy levels dependent upon both electron and proton spins. Due to the electrons' large magnetic moment the electrons are almost completely polarized in the magnetic field at temperatures near $1^{0} \mathrm{~K}$. The microwave power applied at appropriate frequencies simultaneously flips the spin of both a proton and an electron, pumping the protons into one state, while the electrons relax to alignment with the field through interactions with the rest of the material. A steady state is reached in which the slow rate of proton relaxation (spin flips through interactions with the rest of the material, toward equilibrium) is matched by the microwave pumping of fresh protons. 


\section{B. Choice of Material}

The polarized proton target for this experiment had to meet more strict criteria than other experiments require. These were (1) withstand the ionization due to the high intensity electron beam, (2) have a comparatively high fraction of free protons (hydrogen) and (3) able to reverse polarization rapidly. Table II gives the relevant numbers for many'types of materials.

(1) Since the cross section for electromagnetic scattering is so small $\left(\sim 10^{-30} \mathrm{~cm}^{2}\right)$ an extremely intense beam of electrons is necessary to detect a sufficiently large number of scattered particles. This ionizing beam can do two different types of damage to the target. (a) Destroy the free radical which supplies the free electrons to do the Dynamic Orientation (b) Create paramagnetic centers in the target which engage in spin flips with the protons, depolarizing the target. As seen from Table II our typical beam of $2 \times 10^{12}$ electrons/second would totally destroy the LMN crystal in a few minutes, which would make a pleasantly short but unproductive experiment. Figure 9 shows the butanol target polarization as a function of total electron beam through it. The repetition in the pattern is a consequence of our ability to anneal 28 out the paramagnetic centers of $(b)$ above by raising the temperature to $135^{\circ} \mathrm{K}$ (above a phase transition) for 10 minutes. This was of enormous value in extending target life and was one of the keys to our success. The annealing process could not reverse the losses of free electrons (a) and thus the target eventually died of electron failure. (2) We cannut separate kinematically those events which are scatters from free protons as opposed to those scatters from nucleons bound within 
TABLE II

Comparison of target materials by their figure of merit FM for

experiments where (1) free proton events are kinematically

separable $\left(F M=P \sqrt{H_{F}}\right)$ and (2) they are not separable $\left(F M=P H_{F}\right)$.

\begin{tabular}{|c|c|c|c|c|c|c|c|}
\hline Material & Polarization & $\begin{array}{c}\text { Fraction } \\
\text { of Free } \\
\text { Protons } \\
\quad \mathrm{H}_{\mathrm{F}} \\
\end{array}$ & $\begin{array}{c}\text { Polarization } \\
\text { Reversal } \\
\text { Time } \\
\end{array}$ & $\begin{array}{l}\text { Radiation } \\
\text { Damage } \\
\end{array}$ & $\begin{array}{l}\text { Anneal- } \\
\text { able }\end{array}$ & $\begin{array}{l}(1) \\
\sqrt[P]{H_{F}}\end{array}$ & $\begin{array}{l}(2) \\
\mathrm{PH}_{\mathrm{F}}\end{array}$ \\
\hline $\operatorname{IMN}^{29}$ & .50 & .03 & $20 \mathrm{~min}$. & $10^{12}$ & -- & .09 & .015 \\
\hline $\begin{array}{l}\text { Butyl } \\
\text { alcohol }\end{array}$ & .35 & .14 & $30 \mathrm{sec}$ & $4 \times 10^{14}$ & yes & .13 & .05 \\
\hline Glycol 3. & .45 & .10 & $30 \mathrm{sec}$ & $4 \times 10^{14}$ & no & .14 & .045 \\
\hline $\begin{array}{l}\text { Ethyl } \\
\text { alcohol }\end{array}$ & .22 & .14 & $5 \mathrm{sec}$. & $5 \times 10^{14}$ & $?$ & .08 & .031 \\
\hline \multicolumn{8}{|c|}{$\begin{array}{l}\text { a Number of minimally ionizing particles } / \mathrm{cm}^{2} \text { to decrease polarization } \\
\text { to } 1 / \mathrm{e} \text { of its initial value. }\end{array}$} \\
\hline
\end{tabular}


POLARIZATION VS. CHARGE

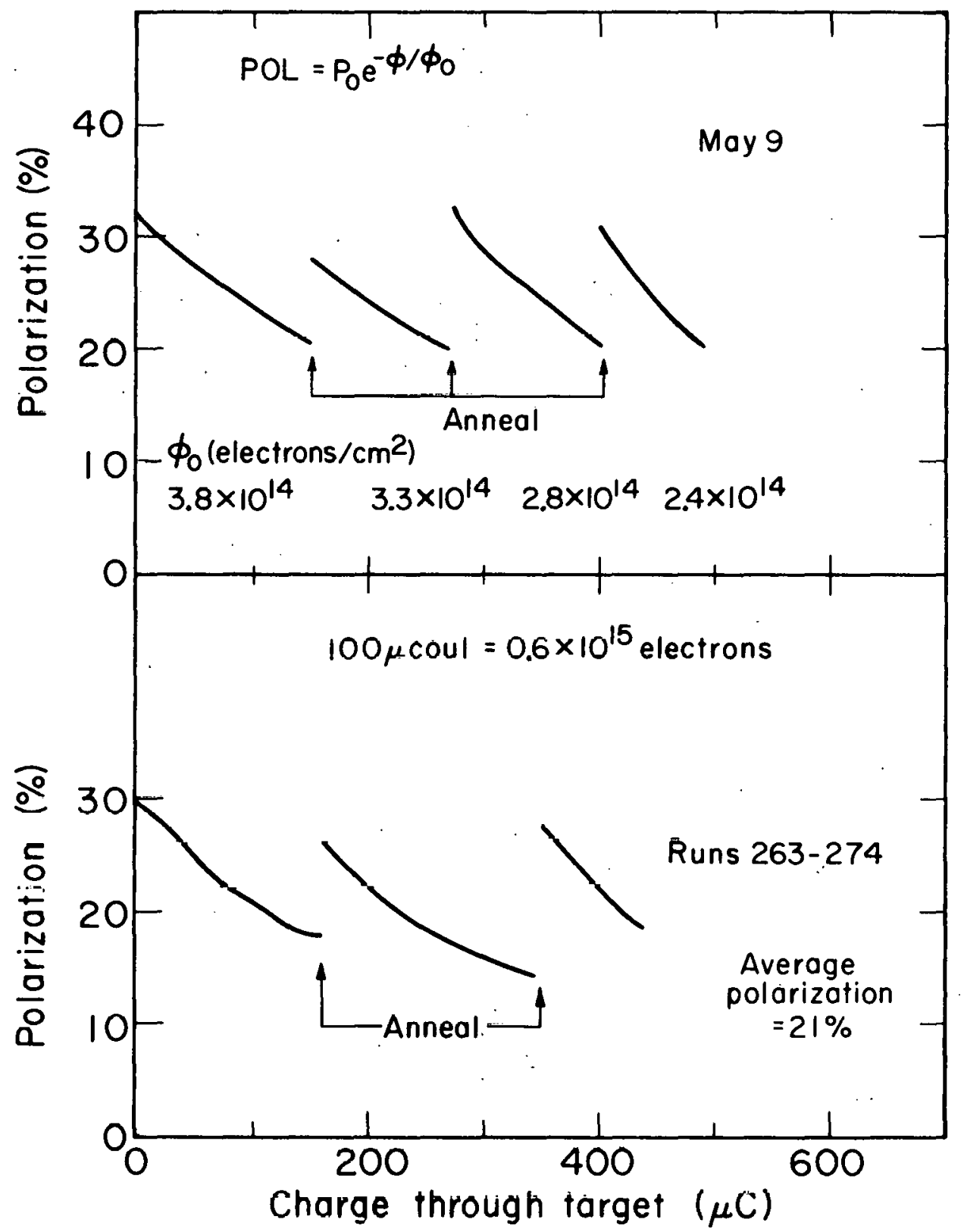

$X B L 6911-6281$

Fig. 9. Radiation damage. Target polarization as a function of electrons passing through the target. The target is about $6.5 \mathrm{~cm}^{2}$ in cross section. The periodic increases in polarization are due to an annealing process described in the text. Approximately $4 \times 1014$ electrons/ $\mathrm{cm}^{2}$ reduce the polarization to $1 / \mathrm{e}$ of its initial value. 
nuclei: Because there is a broad spectrum of missing-mass values the presence of Fermi momentum, while it shifts the calculated missing mass from the true value, does not make the event look different from inelastic scattering on a free proton. An exception occurs below the elastic scattering peak, where scattering on bound protons can give some events that are kinematically different from any scattering on free protons. Even the presence of resonance peaks in the missing-mass spectrum from hydrogen does not help much to distinguish scattering off hydrogen because the resonance widths are generally comparable to the effect of the Fermi momentum on the calculated missing-mass value. The only relevant quantity we can know is the fraction of the total detected events which come from hydrogen $\mathrm{H}_{f}$ (see section VIII and Eq. III-I). The counting-statistics error in $E$ is given by $\sqrt{N} / N$ where $N$ is the total number of events counted and hence $\Delta \mathrm{A}=\left(\mathrm{PH}_{\mathrm{f}} \sqrt{\mathrm{N}}\right)^{-1}$, from $\mathrm{Eq}$. III-3. This can be compared to other experiments where the eeparation of free and bound scatters is kinematically possible. There the number of separable free scatters is $\mathrm{H}_{f} \mathrm{~N}$ and the hydrogen fraction in Eq. III-3 is I for this exclusive sample. Hence $\triangle A=\left(P \sqrt{H_{f} N^{N}}\right)^{-1} \cdot P_{f}$ and $P \sqrt{H}_{f}$ are figures of merit of targets for the two types of experiments and are shown in Table II (we are of course only interested in $\mathrm{PH}_{f}$ for this experiment). (3) To cancel possible random errors frequent polarization reversals are manditory, as was shown in Section III. Hence, to avoid lost time while reversing polarization, a target must not be sluggish when the microwave frequency changes to initiate polarization reversal. At the time the experiment began only LMN, ethanol and butanol 
targets had been developed. We chose a butanol target ${ }^{30}$ (95\% butal alcohol, $5 \%$ water solution saturated with an additional $2 \%$ porphyrexide--a free radical) over the others for reasons which are obvious from Table II. Ethyl alcohol targets were judged unsatisfactory on the grounds of low and unreproducable polarization. (average $22 \%, \max 30 \%$ ) even though they have a slightly higher radiation resistance then butanol. It is not known whether ethanol has a phase transition similar to that in butanol. The polarization of a butanol target is inversely proportional to the absolute temperature at our low temperatures. Hence it is important to dissipate the heat input from the microwaves and beam.

Despite this seemingly endless list of possible targets to choose from, our choice, butanol, while adequate to do the experiment, was not the substance that we had dreamed of as ideal. Only a person wishing to be a hermit in a large city would use it as a perfume; 33 it. is a very poor conductor of heat at low temperatures 34 making it essential to have a large surface-to-volume ratio, i.e., small pieces no more then $2 \mathrm{~mm}$ in diameter; it is a liquid at room temperatures so it is a nuisance to prepare samples with large surface-to-volume ratio. (See Appendix C for problems of heat input to target.) Compounding the above difficulties in target preparation was the inability of our target to withstand more than one days dose of radiation, even with the benefits of annealing. Thus the target had to be replaced by a new one at least once a day. An entire generation of graduate students were trained in this highly useful art and will remember it with tears in their eyes for the rest of their lives despite an apparent lack of job opportunities for butanol target preparers. 


\section{$-53-$ \\ C. Target Preparation}

The first uses of butanol targets (in low beam intensities) did not involve frequent target changes and thus the experimenters could use a rather slow and time-consuming method of preparing a large surface-tovolume ratio. The liquid target solution was placed, drop by drop into liquid nitrogen where it floated around awhile before freezing and dropping to the bottom. About 500 drops later, the: pellets were collected and quickly poured into a pre-cooled cryostat cavity which was then put together and cooled before the alcohol could melt. Precautions had to be taken to prevent frost from forming on the cold cryostat which might clog narrow tubes and form a hydrogen-rich unpolarized ice block which could distort the effective polarization of the target. This process has the advantage of providing free flow of liquid helium to all parts of the target and having little extraneous material in the beam, but is too involved for a daily procedure.

Instead, small cylindrical nonhydrogenous plastic F.E.P. bags 35 were made to contain the polarizable fluid. The plastic was made thin, ( $1 / 2 \mathrm{mil}$ ) to avoid excess material in the target. Fach bag was about 8" long and had 8 narrow ribs which, when filled with alcohol and sealed, were about $2 \mathrm{~mm}$ in diameter. The bags were folded accordion style to form a $1 " \times 1 " \times 1 / 4$ " semi-solid block which had narrow channels between the ribs to allow liquid helium to flow through and do its cooling. Six such bags constituted one target. It is a well-known fact that $1 / 2-m i l$ plastic bags with 8 sealed ribs, after being loaded with 12" Iong hyperdermic needles by shakey-handed graduate students staggering from the 


$$
-54-
$$

alcohol stench and rushing to avoid deterioration of the free radical while it is warm, leak. If such a bag were to be placed in a vacuum (normally maintained in the cryostat for reducing the vapour pressure of the liquid He) before the alcohol froze this small leakage would turn into a disasterous rout. To avoid this, the loaded cryostat was cooled to below the alcohol freezing point at atmospheric pressure before our big vacuum pumps were turned on. Unfortinately, target preparations was more of an art than a science and we gradually concluded that Jacques Louis David would have been no more successful than Jackson Pollock. We were not able to evolve a procedure of target mixing, rib injection and cooling which gave consistant values of polarization from day to day. Polarizations varied between $35 \%$ and $25 \%$ and especially $10 \mathrm{w}$ polarizations were deemed unsatisfactory necessitating a second target change.

The alcohol mixture was prepared in a relatively clean environment by dirty people. Both the alcohol and the distilled water were deoxigenated by bubbling $\mathrm{N}_{2}$ through them. Two percent porphyrexide was added to a mixture of $95 \%$ alcohol and $5 \%$ water by weight and mixed usinf a magnetic mixer. Undesolved lumps of porphyrexide were allowed to settle to the bottom and the pure sparkling stinking red beverage was then decanted. (Lumps of porphyexide would not contribute to the polarization and would absorb large amounts of microweve powcr and thus heat up the target.) The brew was then mainlined into the veins of the bag with a 12": hyperdermic needle borrowed from a friend on Telegraph Avenue. Aside from several graduate students and one plastic bag developing hepatitis and dying, the senior researchers considered this an effective technique. 
$-55-$

Target Control

Once appropriate tuning was done manually, the target was controlled during data taking by a PDP-5 computer. On command from the main SDS-9300 computer to change polarization, the PDP-5 would change the D.C. bias on the power supply of microwave generator, thus changing the frequency to that appropriate to the new polarization. The PDP-5 also controlled the readout of polarization value and reported the numbers to the SDS and the people. 


\section{Cryostat}

For the target to function, it must be in a high uniform magnetic field ( $25 \mathrm{KG}$ ) and be at an extremely low temperature $\left(I^{\circ} \mathrm{K}\right)$ and be bathed by microwaves $(70 \mathrm{GHz})$. The high magnetic field and low temperature increase the separation of the magnetic energy levels relative to the Boltzmann energy $\mathrm{KT}$ and hence increase the polarization. The magnetic field of $25 \mathrm{kG}$ is determined by matching to our already existing (for IMI targets) microwave equipment and is at the upper limit of iron-core magnet possibilities. $I^{\circ} \mathrm{K}$ is the lower practical limit on temperature using liquid $\mathrm{He}^{4}$ at low pressure as the coolant. Either halving the temperature (using $\mathrm{He}^{3}$ ) or doubling the magnetic field is expected to double the polarization of butanol and laboratories around the world are developing such advanced targets. 36

To obtain the high magnetic field a specially designed $\mathrm{C}$ magnet named Zoltan was constmucted. After shimming the cobalt-iron (Hyperco27) pole tips, the magnet met the requirements of uniform field ( 1 in $10^{4}$ ) to insure uniform energy separation over the entire target of $1 " x 1^{\prime \prime} \times 1.6 "$. The gap was $3 "$ between the 8"-diameter tapered pole tips. The open area was $270^{\circ}$ in the plane of the pole tips and $\pm 20^{\circ}$ perpendicular with the cryostat mounted. This was more than adequate for this experiment where we used only $3^{\circ}$ and $1^{\circ}$, respectively:

The cooling 1s accomplished by putting the target in a, small can filled with liquid helium and then using vacuum pumps to reduce the vapor pressure to a few hundred microns which corresponds to $1^{\circ} \mathrm{K}$. of course there are some problems involved in (1) insulating thc system, (2) pumping off 4800 cubic feet of He gas/minite, (3) refilling the can 
as the He boils away. Since liquid He is expensive, practical considerations of not wasting its cooling power had to intrude on our pure, abstract scientific minds.

An entirely new cryostat (for our group) was used for this experiment which provided a continuous flow of helium to the can, the ability to warm up and cool down the can rapidly (for annealing), ease of quickly gaining usable access to the can to change targets every day and minimal nontarget material in the beam line: The design was basically a larger version of that of Roubeau 37 and is shown in Fig. 10.

The can which holds the target in the cryostat serves as a liquidhelium container, microwave cavity and Nuclear Magnetic Resonance (NMR) coil. The can is suspended in an inner cylindrical vacuum chamber at the end of a cylindrical wave guide which brings in the $4 \mathrm{~mm}$ microwaves to do the polarizing. This cani,mustrheret thin.windowsi forwthè tir passage of the beam, have high electrical conductivity so as not to absorb too much of the microwave power, and a top which presents no barrier to the boil-off He gas and at the same time prevents the microwaves from leaking out. We used 3 -mil aluminum plated with 0.2 mils copper to make a can 1-1/4" high, 2-1/2" long, and 1.6" along the beam line. The target was centered in the can. The top was a 1-1/2-mil-thick copper screen with 4-mil square holes (well below cut off for our $4 \mathrm{~mm}$ microwaves but giving 35\% transparency for the He gas). Measurements indicated no pressure drop across the screen. This screen was soldered to the top of the can and was easily removed for the insertion and removal of new targets (the half life of a screen was about 2 target changes). To make a rectangular can into an $\mathrm{NMR}$ coil required an 
$-58-$

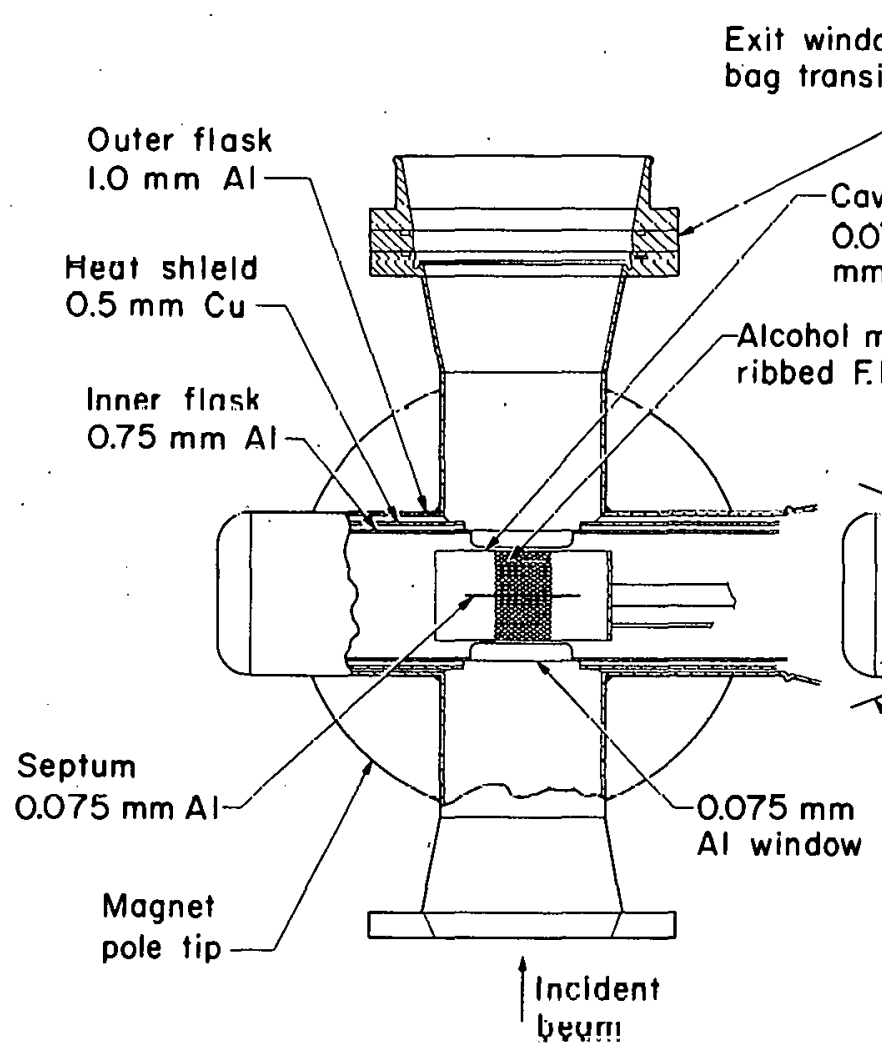

(a)
0.075

$\mathrm{mm} \mathrm{AI}$

F.E.P. bags

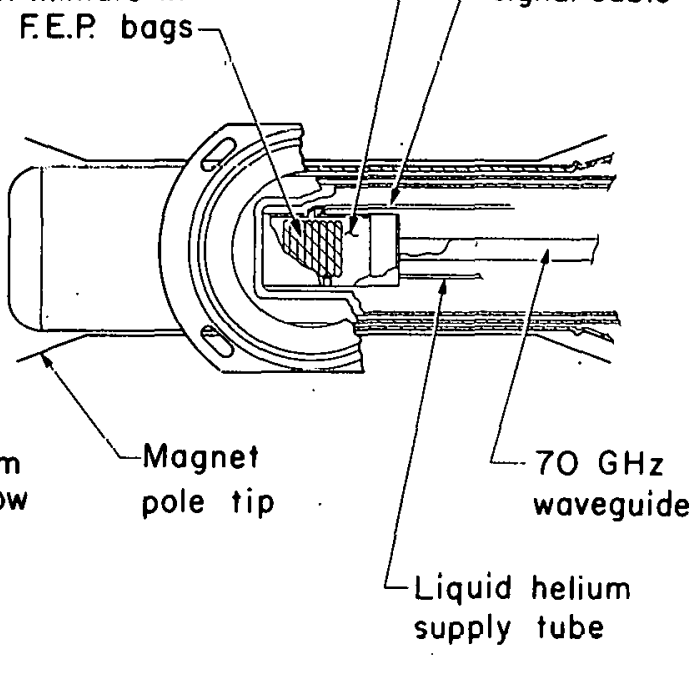

(b)

XBL702-2353

F1g. 10. The cryostat. (a), Top view, partially cut away, uf lhe polarized proton target inside the magnet gap. (b) View looking along the incidenl beam line at the targct, partially elut. A.WAy. 
additional conducting fin (septum) to carry the return current and also act as a magnetic field guide. The current and magnetic-field distribution are shown in Fig. 11.

The evaporated helium gas from the can cools the back ends of the cryostat and heat shields befo it reaches the pumps. Liquid helium coming from the dewar in a long transfer line first enters a phaseseparating chamber where gas formed due to heat leaks in the transfer line is removed and used to cool the heat shields and back ends of the cryostat. The liquid then passes through a heat exchanger where it is cooled by the outgoing boil-off helium gas from the can. It is cooled well below the lamda point, to about $1.6^{\circ} \mathrm{K}$. Once it is superfluid the helium easily passes through a small needle valve into the can which is at about $I^{\circ} \mathrm{K}$ and 100 microns pressure. There it boils off slowly due to heat entering the can from assorted tubes, waveguide cables, beam, and microwave power. The adjustable opening in the needle valve controls the liquid helium flow to the can and the heat input to the cavity controls the boil-off rate. It is estimated that the heat load from other than the microwave power is very stable, depending only on things like room temperature. Variations in microwave power, expecially when the target polarization is reversed (change of microwave frequency), do occur and are partially compensated for by a stabilizing feedback. The more helium:boiled off at the can the more gas is available to cool off the back of the cryostat and lower the temperature of the heat shiel.ds and thus reduce the heat input from other sources. We found the operation of the cryostat remarkably stable, requiring no adjustments over 


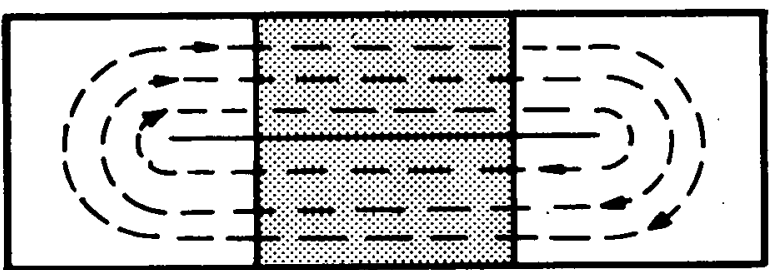

$105.5 \mathrm{Mc}$

Readout magnetic field

\section{Target}
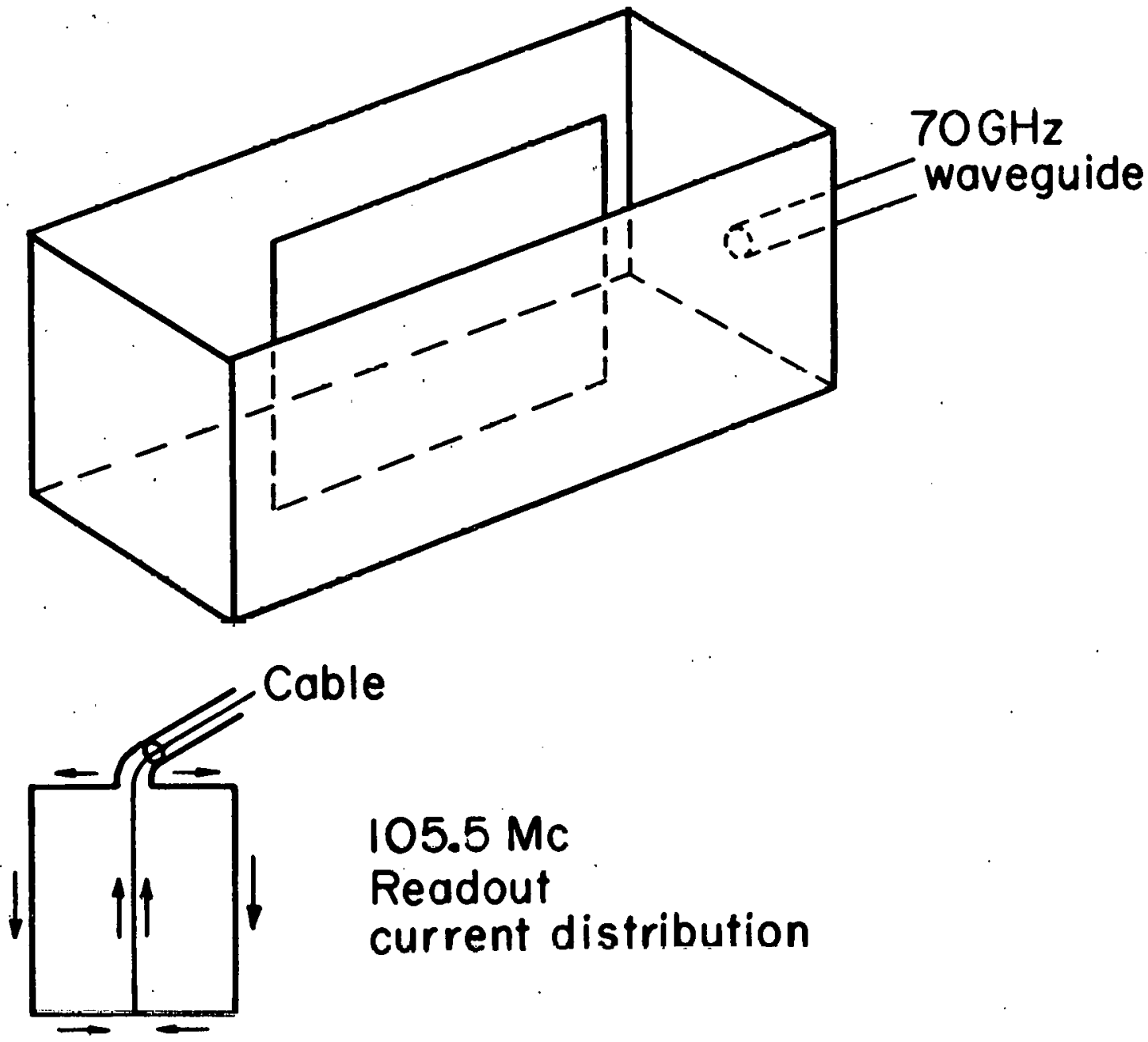

XBL706-3206

Fig. 1l. The can, showing the target position, magnetic field lines and current distribution from the MMR system. 
$-61-$

periods of many hours. To insure that there was no fluctuations in the helium level in the can (which would be the equivalent of fluctuations in target density--the material in the beam is $10 \%$ liquid $\mathrm{He}$ ) we always ran at a higher helium flow than strictly necessary, with liquid presumably spilling out over the top. In this mode the cryostat operated continuously all day without need for adjustment. Measurements of helium consuption with and without microwave power, indicate a heat leak of about 0.75 watt and microwave power of about 0.4 watt.

Operation was monitored by several indicators. (1) The pressure above the can was measured by a Hastings guauge. (2) The temperature at various places within the cryostat was monitored by carbon-resistance thermometers. The resistance is an exponential function of $1 / T$ at very low temperatures (our region of operation) which provides a very sensitive measurement of temperature. The resistors, even when shielded in copper cases, were warmed by the small amount of microwave power which leaked through the screen. The resistors were thus useful only as a relative measures of temperature during polarized operation. (3) The gas flow from the can boil off (main flow) and the gas flow from the phase separator were also measured.

Table III shows some pertinent numbers on the normal operation of the cryostat. Of note is the exceedingly large loss of helium from the phase separator at the end of the transfer line. This heat loss was found to be proportional to the length of the transfer line. Unfortunately the fixed experimental arrangement at SLAC required an exceedingly long (6-foot) transfer line and we were forced to take our loses and smile. 
TABLE III

\section{CRYOSTAT OPERATIONS}

Definitions and constants

Flow $: 1$ standard $\mathrm{ft}^{3} / \mathrm{min}$ He gas $=2$ liquid liters $/$ hour

Heat of vaporization : ( He at $4^{\circ} \mathrm{K}$ ) $22 \mathrm{~J} / \mathrm{gm}=2.9 \times 10^{3} \mathrm{~J} /$ liquid liter

Power input $: 1$ watt creates flow of $.65 \mathrm{SCFM}=1.21$ /hour

\section{OPERATIONS}

Flow (main)

.65 SCFM ( 1.3 liquid liters/hour)

Flow (separator)

2.0 SCFM ( 4.0 liquid liters/hour)

Pressure (can) $150 \mu$

Temperature (can)

$1.02^{\circ} \mathrm{K}$

Temperature (front heat exchanger)

$1.9^{\circ} \mathrm{K}$

Temperature (back heat exchanger)

$2.8^{\circ} \mathrm{K}$

Temperature (heat shield)

$18 \quad O_{\mathrm{K}}$

Helium to cool down to room temperature

Cool down time to stable operation

Helium use per 16 hour day (including cool down)
. 25 liters

$1-1 / 2$ hours

100 liters
Power Input: microwaves off micruwaves un microwave power input
.45 SCFM $=.75$ watt

$.65 \mathrm{SCFM}=1.1$ watt

.35 watt 
$-63-$

Almost all of the helium gas was recovered and pumped to a liquifying unit about $1 / 5$ of a mile away. 
E. Measurement of Target Polarization

The usual technique of Nuclear Mangetic Resonance (NMR) is employed with special sophistication. Our target is large and it is necessary to measure the average polarization of the entire target as seen by the beam. The detection rf field must be uniform throughout the target and not large enough to depolarize the target. Consequently the signals we deal with are quite small and carefully designed equipment is needed to avoid noise problems.

The MMR technique involves stimulating proton spin flips with a.n applied rf magnetic field of the appropriate frequency. Let $g(\omega)$ be the line shape function for the proton resonance, i.e. the probability that the transition will be observed with the frequency $\omega . g(\omega)$ is thus the distribution of relative proton energies. Therefore we normalize $g(\omega)$ by the condition:

$$
\int_{-\infty}^{\infty} g(w) d w=1 .
$$

An rf magnetic field applied perpendicular to the main field will flip some protons. Let the $\mathrm{rf}$ field be $\mathrm{H}=\mathrm{H}_{1} \cos (\omega t)=\operatorname{Re}\left(\mathrm{H}_{1} \exp (i \omega t)\right)=$ Re(He) Then the transition probability per unit time at frequency $\omega$ is

$$
W=\frac{\pi}{2}\left(\gamma H_{1}\right)^{2} \rho(w)
$$

where $\gamma$ is the free proton gyromagnetic ratio and $\rho(\omega)$ is the final state density in energy $=\delta(\omega)$ for one state. If $\Delta N(\omega)$ is the difference in population of protons along and opposite the main field with energy difference between $\omega$ and $(\omega+d \omega) k$, and $\Delta N$ the total population difference then:

$$
\Delta \mathbb{N}(\omega)=g(\omega) \Delta v i d \omega
$$


Let $\mathrm{N}$ be the total number of protons. Then the power absorbed at frequency $\omega$ is:

$$
\Delta \mathrm{N}(\omega) \mathrm{Whw}=\Delta \mathrm{Ng}(\omega)+\frac{\pi}{2}\left(\gamma \mathrm{H}_{1}\right)^{2} \text { hw }
$$

where we have used $\delta(\omega) d \omega=1$.

Remembering that the polarization $\mathrm{P}=\Delta \mathrm{N} / \mathrm{N}$ we can write:

$$
\text { Power absorbed }=\sin \frac{\pi}{2} g(\omega)\left(\gamma \mathrm{H}_{1}\right)^{2} \text { hwNP . }
$$

The power absorbed can be related to a complex susceptability of the material $X=X^{\prime}-i X^{\prime \prime}$ where the magnetization density is given by $M_{c}=X H_{c}:$
$\quad M=\operatorname{Re} M_{c}=\operatorname{Re} X H_{c}=\operatorname{Re} X H_{1} e^{i \omega t}=X^{\prime} H_{1} \cos \omega t+X^{\prime \prime} H_{1} \sin \omega t$. Then the average power abosrbeditunit volume $=$

$$
\begin{aligned}
-\vec{M} \cdot \frac{d \vec{H}}{d t} & =4 \omega H_{1}^{2} X^{\prime}\langle\sin (\omega t) \cos (\omega t)\rangle_{a \cdot v}+4 \omega H_{1}^{2} X^{\prime \prime}\left\langle\sin ^{2}(\omega t)\right\rangle / a v \\
& =2 \pi H_{1}^{2} X^{\prime \prime}
\end{aligned}
$$

Thus equating the two expressions for the power absorbed

$$
P=\frac{4 \chi^{\prime \prime}(w)}{\pi \hbar \gamma g(w) N_{v}}
$$

where $\mathbb{N}_{\mathrm{v}}$ aris the number of protonsłunit volume. This implies that:

$$
x^{\prime \prime}(\dot{\omega}) \% g(\omega)=\text { constant for a given target polarization }=P C
$$

where $a:=j \operatorname{ri} \gamma \mathrm{N}_{\mathrm{v}} / 4$

Thus: $\quad x^{\prime \prime}(\omega)=P C g(\omega)$

$$
\int_{-\infty}^{\infty} X^{\prime \prime}(w) d w=P C \int_{-\infty}^{\infty} g(w) d w=P C
$$


Hence

$$
\int_{-\infty}^{\infty} x^{\prime \prime}(w) d w
$$

is proportional to the polarization and independent of the line shape $g(\omega)$.

Since the constant $\mathrm{C}$ is not easily determinable, nor are the normalization parameters in the circuit exactly known, we use a procedure of measuring the ratio between a known and unknown polarization. First a measurement $\cdots$ le of the thermal equilibrium (TE) or natural signal is made. When there is no microwave pumping the target is in thermal equilibrium with the Iiquid He and the polarization is given by Eq. V-I providing carc is taken to wait long enough for equilitibrium to be established. The temperature is measured with calibrated carbon resistors. Typically the temperature is $1.05^{\circ} \mathrm{K}$ and the TE polarization is about $0.24 \%$. Next a measurement of the signal is taken with the target polarized and the ratio of the two $\mathbb{R M R}$ readings is the ratio of the two polarizations.

To measure $X^{\prime \prime}(\omega)$ we use a tuned circuit. The can containing the target acts as a one-turn coil which surrounds the sample whose $X^{\prime \prime}(\omega)$ we wish to measure. An extremely quiet rf oscillator throttled by a high impedance acts as a constant current souree. The rest of the circuit is show in Fig. 12. The cable which extends from the cavity to the irif oscillator is 2 wavelengths long. Being an integral number of half wave lengths, this cable does not introduce any capacitance or inductance. The $\mathrm{R}$ represents the effect of cable resistance (stainless steel to avoid heat conduction) and stray resistance, $\mathrm{I}$ is the inductance 


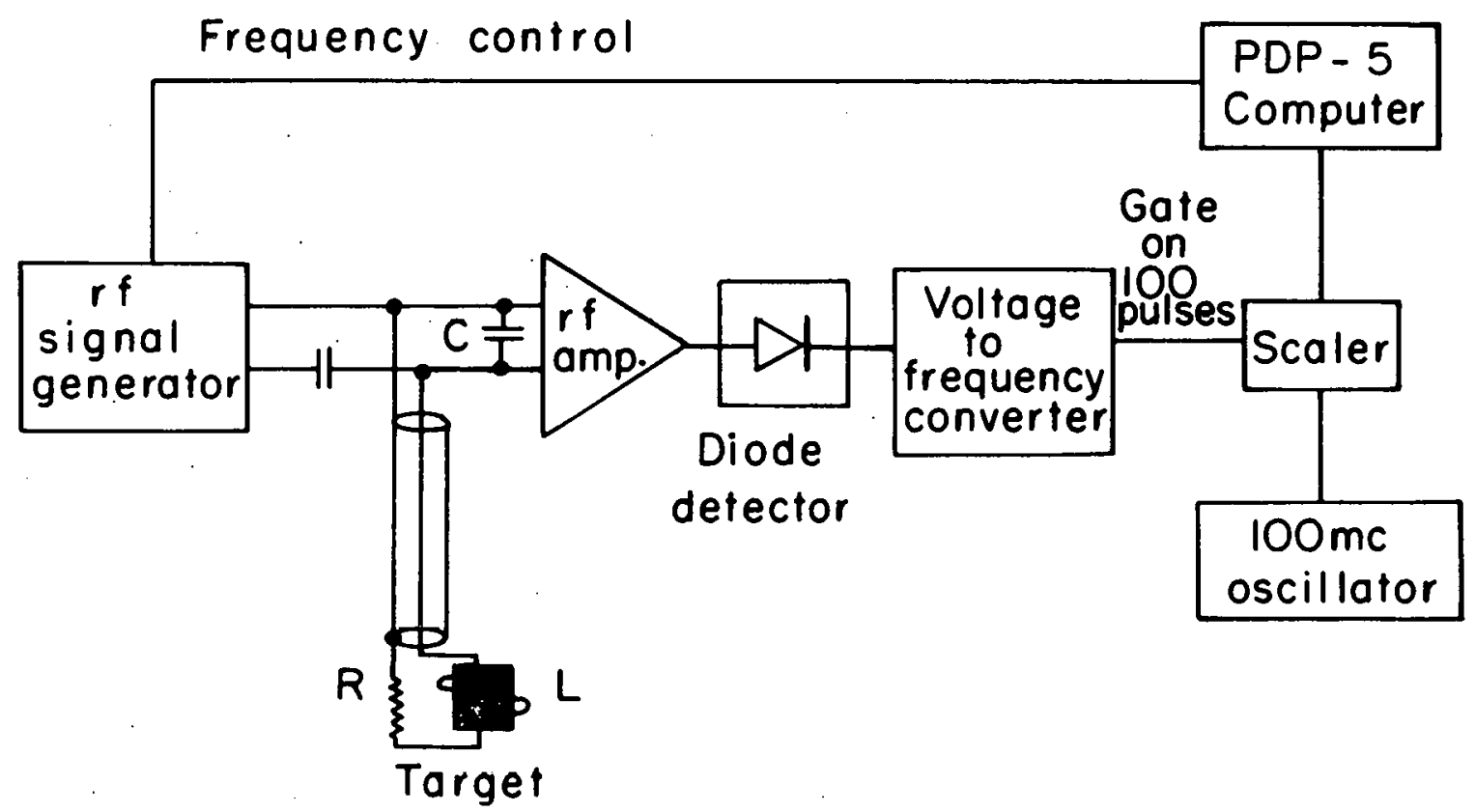

XBL707-3434

Fig. 12. Target Polarization Nuclear Magnetic Resonance Circuit. 
of the coil and $C$ is an adjustable capacitance to tune the circuit. It is shown in many places ${ }^{29}$ that the absolute value of the impedance across $\mathrm{C}$ is

$$
|z|=(\omega L)^{2} /\left(R+\varphi^{\prime \prime}\right)
$$

where $\varphi^{\prime \prime}=4 \pi \eta \omega L X^{\prime \prime}$ and $\eta$ is the filling factor (fraction of the rf magnetic field energy that is within the target material), and $\omega$ is the frequency, assumed near the circuit resonant frequency. This relation is true only at the circuit resonance and thus to avoid complicated corrections we. arrange that our readout electronics 38 automatically retunes the circuit (by adjusting a capacitance parallel to $C$ ) as $\omega$ is changed (called tracking). To find the polarization which is proportional to

$$
\int_{-\infty}^{\infty} \chi^{\prime \prime}(w) d w
$$

we measurc

$$
\frac{1}{|Z|}=\frac{R+Q^{\prime \prime}}{(\omega L)^{2}}
$$

and then subtract the "background"

$$
\frac{1}{\left|Z_{0}\right|}=\frac{R}{(\omega L)^{2}}
$$

by taking the same measurement with $\omega$ far from the proton resonance (where $X^{\prime \prime}=0$ ). This background is about $10^{3}$ times the signal when the polarization is $0.24 \%$ for TE signals. The area of the signal

$$
\int_{-\infty}^{\infty}\left(\frac{1}{|z|}-\frac{1}{\left|z_{0}\right|}\right) d w
$$

is then proportional to 


$$
\int_{-\infty}^{\infty} x^{\prime \prime}(w) d w
$$

In practice a PDP-5 computer takes a measurement at a given frequency $f_{i}$ at proton resonance and background in a pattern of (I) proton resonance region $R_{i}^{I}$ (at $\left.f_{i}\right)$, (2) high frequency backgfound $B_{i}^{h}\left(\right.$ at $\left.f_{i}+200 \mathrm{kHz}\right)$, (3) low frequency background $B_{i}^{L}\left(\right.$ at $f_{i}-200 \mathrm{kHz}$ ) and (4) proton resonance region $R_{i}^{2}$ (at $f_{i}$ ). The computer steps through the resonance in this manner in $2-1 / 2 \mathrm{kHz}$ steps.

$$
\frac{1}{2} \sum_{i}\left[R_{i}^{\prime}-\left(B_{i}^{i}+B_{i}^{h}\right)+R_{i}^{2}\right]
$$

is then proportional to

$$
\int_{-\infty}^{\infty} \dot{\chi}^{\prime \prime}(w) d w
$$

and thus to P. Both background terms are measured to try to cancel the slight linear frequency dependence of the circuit. A complete readout of polarization took 1.2 seconds.

When measuring very small polarizations this deviant behavior is often large enough to make the inaccuracies in this procedure a substantial fraction of the signal size. In this case we repeat the same procedure with the magnetic field about $250 \mathrm{G}$ (1\%) higher or lower to get entirely out of the proton resonance region. The result of this "off field" measurement is thus a measurement of the circuit characteristics which depend only on the readout frequency and not on the magnetic field. This circuit background (which can be either positive or negative) is then subtracted from the "on field" measurement to give the actual area of the target proton resonance. By this procedure we can reproducibly measure the thermal equilibrium signal to within $3 \%$ of itself 
$-70-$

at various temperatures and various times. This is what limits the accuracy with thich we know the target polarization. In this experiment, of course, the target polarization is a normalization factor and since we are looking for a deviation from zero this error is negligible for our purposes.

To convert the analog signal (which is a voltage proportional to the impedance $V=Z I_{0}, I_{0}$ the constant current) to a digital representation of

$$
\frac{1}{|Z|} \propto \frac{1}{N \mid}
$$

the $n f$ voltage is first amplified and then rectified by a diode. The diode circuit has been measured to give a d.c. output linearly dependent (to $1 \%$ ) on the rf voltage within our operating region. This voltage level output is then converted to a number proportional to $1 / \mathrm{V}$ in external circuitry. As show in Fig. 12 the voltage is converted to a frequerrey. A rixed number of cycles llen dels ds a yale vil a fasl scalen recording signals from a stable $100 \mathrm{MHz}$ pulse generator. This scaler reading, inversely proportional to the voltage as desired, is easily read by the computer. This circuitry has to be accurate to 1 part in $10^{5}$ to allow for the large subtractions. Second-order corrections to the measured polarization due to dispersive effects of the real part of the inductance has been calculated to be less than $2 \%$.

Before the experiment, when the PDP-5 computer was not available to control the above readout procedure, we used an oscilliscope to measure the diode output directly, with a simple circuit to simulate the background. The size of the signal (background subtracted)was typically 
$700 \mu \mathrm{V}$ for $0.24 \%$ polarization and about $100 \mathrm{mV}$ for $35 \%$ polarization while the background was $700 \mathrm{mV}$. If we assumed that the line shape $\mathrm{g}(\omega)$ was independent of polarization then we could quickly obtain the polarization by measuring the maximum voltage in the signal. What we actually want is:

$$
\frac{1}{|z|}-\frac{1}{\left|Z_{0}\right|} \propto \frac{1}{|V|}-\frac{1}{\left|V_{0}\right|}=\frac{V_{0}-V}{V V_{0}}
$$

If $\mathrm{V}=\mathrm{V}_{\mathrm{O}}^{\mathrm{O}}+\Delta \mathrm{V}$ where $\Delta \mathrm{V}$ is signal size then:

$$
\frac{1}{V}-\frac{1}{V_{0}}=\frac{\Delta V}{V_{0}\left(V_{0}+\Delta V\right)}=\frac{1}{V_{0}^{2}}\left(\frac{\Delta V}{1+\frac{\Delta V}{V_{0}}}\right)
$$

and so knowledge of $\mathrm{V}_{\mathrm{O}}$ and $\Delta \mathrm{V}$ gives one a number proportional to the polarization. By integrating $\frac{1}{V}-\frac{I}{V 0}$ numerically by hand we found that the assumption of unchanging line shape was a valid one. (It is interesting to note that the physical shape of the target with respect to the wiin $25 \mathrm{kG}$ field did affect the line shape. When the target was contained in small cylindrical bags aligned with the field, the polarized protons increased (decreased) the field that neighboring protons experienced when the polarization was along (opposite) the magnetic field and thus increased (decreased) the resonant frequency slightly, but clearly noticeably.)

A more complete description of the polarization readout system is contained in reference 39. 


\section{RUNNIING PROCEDURE}

\section{A. Beam Tune Up}

It was necessary to change targets every day and each target was a separate individual whose physical appearance and geometry (as well as its virtue which was measured in $\%$ polarization) were slightly different every day. Thus not only did we have to refocus and stabilize the electron beam once a day following an 8-hour shutdown to change targets, but to steer it and adjust the vertical and horizontal sweep to approximate by a rectangle the amorphous creature within the cryostat (a task approximating that of squaring of the circle or vice-versa). At the beginning of the experiment the above procedure took almost all of our allotted 16 hours of running a day before the beam was passed on to less fussy users. Our great concern with beam stability was inspired by fear that any instability anywhere would introduce a random error.

Our daily procedure began with the installation of a fresh (and smelly) polarized target. While the cryostat was cooling toward thermal equilibrium at $l^{\circ} \mathrm{K}$, we used the movable beam dump as a target shield and turned on the electron beam. The SLAC crew did a superb job in attempting to reduce the beam position jitter (due to pulsed magnets which were used to divert some pulses to other experiments, balky klystrons, etc.). Unfortunately much was left to the hand of God who this summer was suffering from a mild case of Parkinson's disedse. Our very strict requirements on stability were based on a fear that. misdirected beam pulses would go through portions of the target of different density or miss the target completely. While we were never completely satisfied with the beam position stability, there is no evidence that 
any uncertainties were introduced in our results from this cause. To center the beam on the target we expanded the horizontal and vertical sweep and plotted (by our SDS-9300 computer of course) the number of events vs. sweep position. This target profile was then used to adjust the centering and sweep size. The profile shown in Fig. 13 is somewhat atypical heving been taken with special precautions and procedures. More typically, interpretation of these profiles was more art then science. However the sweep size obtained was almost always very close to the $I^{\prime \prime}$ nominal target size and the center position varied by a few millimeters from the nominal central beam line. An X-ray picture of the target by a photon beam taken during the following experiment is shown in Fig. 14. Unfortunately during this centering procedure the target's virtue was being compromised by the rape of radiation damage. Two computers were used to do the bureaucratic work required. A PDP-5 computer from Berkeley controlled the target as a slave of the SLAC SDS-9300 computer. Information was passed from accumulator to accumulator through two independent buffers (one for each direction). Each computer then saw the other as a simple buffer to be filled or read at its leisure. 


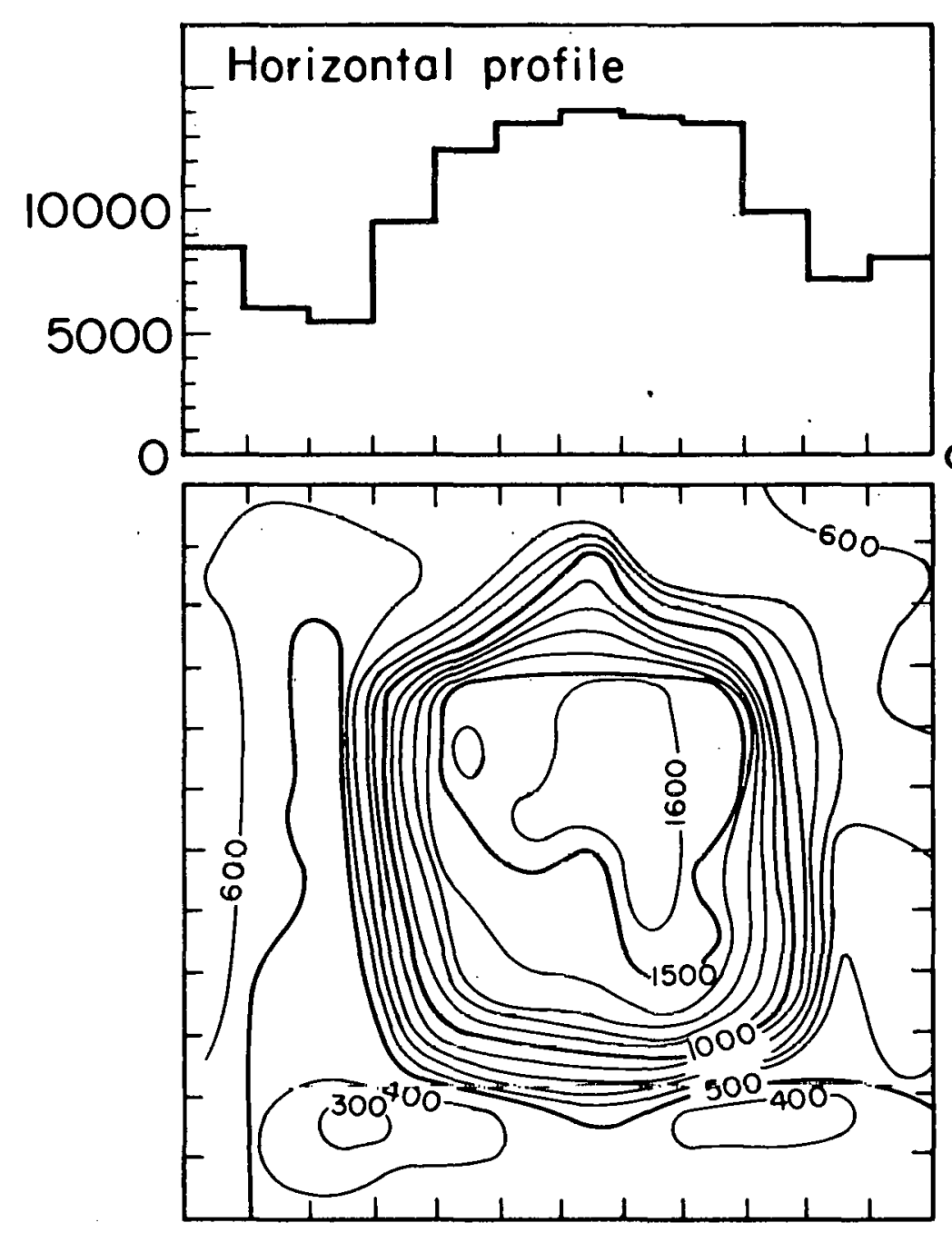

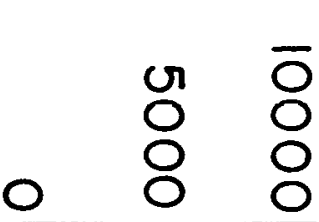

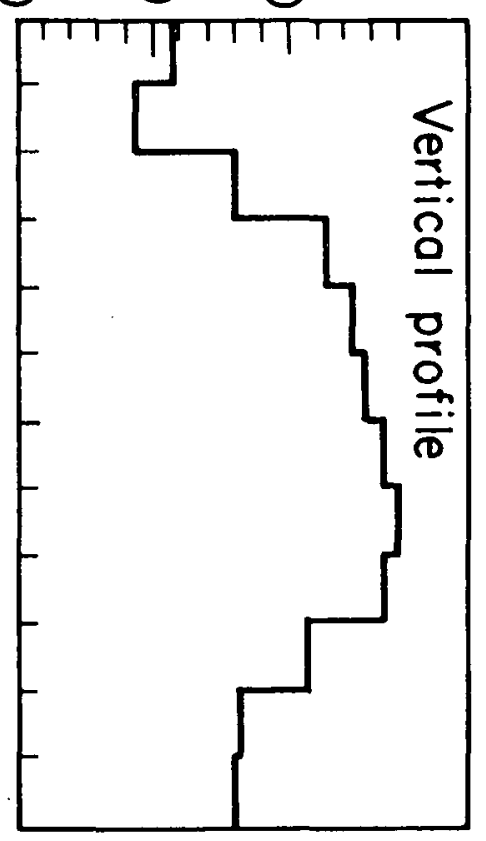

XBL698-345I

Fig. 13. Profiles of polarized target taken with electron beam. 


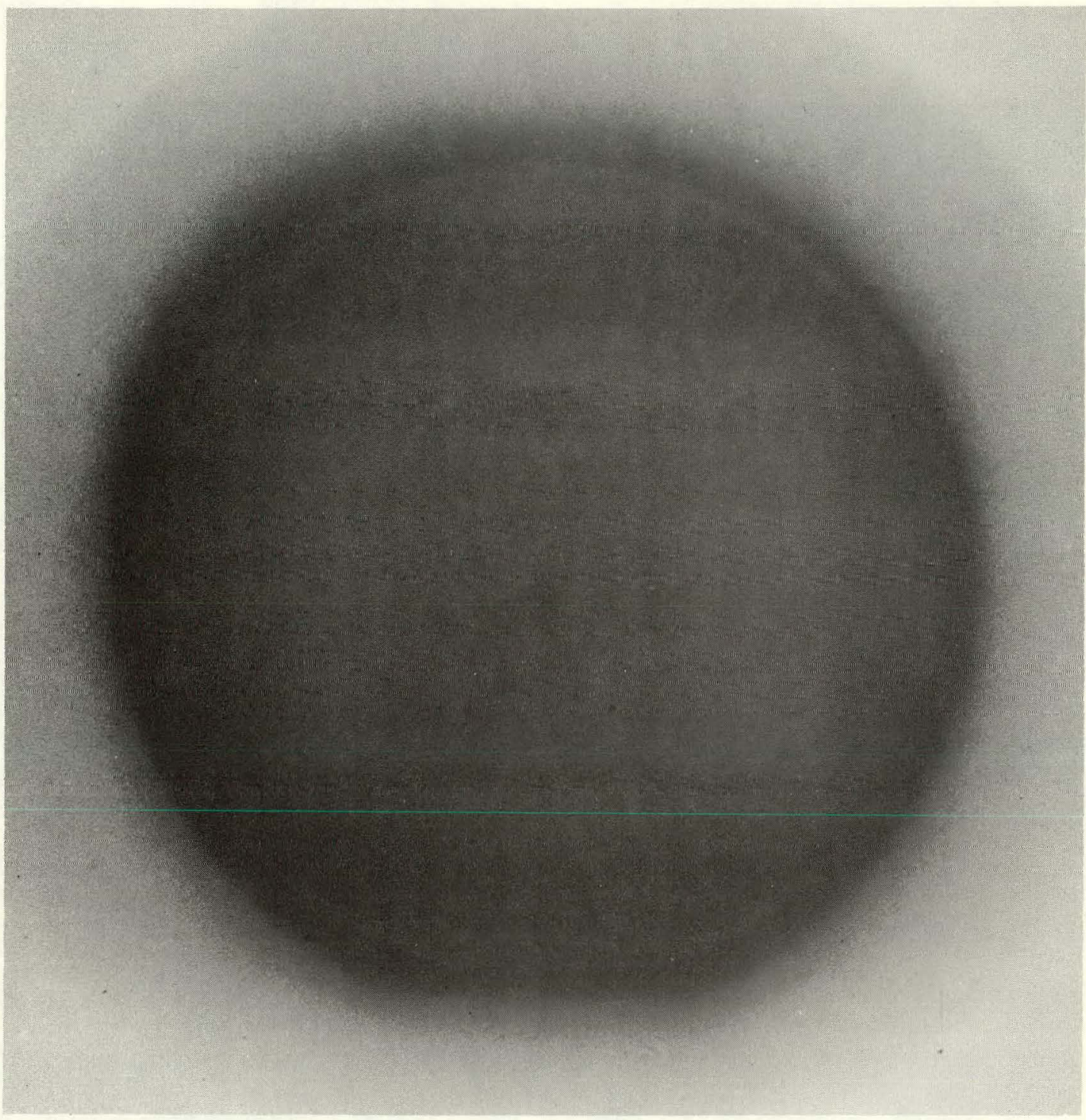

XBB 6910-6804

Fig. 14. X-ray photograph of target taken by the SLAC photon beam. A skilled observer will note the two teflon blocks the target bags sit on, the vertical ribs of the target and the NMR cable at the top. The dark spot in the center is a drop of solder. 


\section{$-76-$ \\ B. Data Taking}

The basic unit of data taking was one complete sweep of the beam over the target. Since the target had a non-uniform density it was essential to include equal amounts of data from all sections of the target for each sign of polarization. Six complete sweeps, taking about 10 seconds, constituted a subrun. The beam monitors as we.71 a.s a.17 the scalers, were read by computer. If the monitors disagreed by more than $4 \%$ or if the target polarization readings did not meet criteria of magnitude and number, the subrun data were rejected from the on-line analysis. After a predetermined number of good subruns (typically 12 or about 2 minutes, called a signrun) the SDS computer turned the beam intensity down to 1 pps (to avoid target radiation damage) and ordered the subservient PDP-5 computer to initiate the target polarization reversal. When the polarization had reversed and reached a preset magnitude (usually $20 \%$, taking about $50 \mathrm{sec}$ ) the SDS computer turned the beam back on and reinitiated data taking. Each subrun, and thus each signrun began and ended when the sweeping beam was on the boundry between the top and middle target regions thus ensuring that the non-uniform target would be sampled by the beam in the exact same manner for each segment of data. This procedure was repeated four times to make a pattern of polarization +-+- or -+-+ which was called a foursum (lasted about $12 \mathrm{~min}$.$) . The data, was analyzed in terms of complete foursums to$ try to balance out noise both for the actual asymmetry calculation and for the "test" asymmetries described below. If a foursum had to be interrupted due to minor disasters such as beam drift or target failure, a new foursum was begun and the incomplete one thrown out. Several foursums 
(usually 4) constituted a complete run. It was only between runs that human hands took over from the computer and made those careful observations that only man is capable of doing (yawn, scratch, and push the start button). The beam position, monitors and trigger rates were then checked, data for the run were printed out by the computer and carefully stored in books, target polarizations were thought about and, if necessary, (every $3-4$ runs, about 3 hours) the target was annealed. When a new run was begun the starting polarization was in the opposite direction to the starting polarization of the previous run to try once again to cancel out any insidious systematic effect. Up-to-the-microsecond information was displayed by the computer on its oscilloscope. Calculated asymmetries as well as missing-mass spectra, event and accidental rates, beam position, target polarization, and over a hundred other numbers and graphs were thus available to anyone who was diligent enough to look. Due to the large size, attractive format and impressive array of buttons as well as the importance of monitoring possible systematic drifts (e.g. a misread scaler or fluctuating beam could easily be detected if looked for) many of us spent continuous hours just staring at the screen.

Typical data taking rates were:

\begin{tabular}{lc|lc}
\hline Pulses per second: & 180 & Triggers/pulse: & 1.5 \\
Beam electrons/pulse: & $1.0 \times 10^{9}$ & Accidentals/trigger: & .012 \\
Root Mean Square polarization & .22 & \\
Our running day ended as the sun came over the pastureland and a
\end{tabular}


$-78-$

begged to return from whence it came and anxious physicists on other beam lines prepared to take over the duties. While the target was being changed, the data taken were reanalyzed off-line, placed on summary tapes, and combined with data taken earlier. In this way we were able to have a preliminary analysis of the data to date available at all times to guide our decisions as to what we should do next (electron or positron beam, beam energy, missing mass, are there any systematic effects). At times the enthusiasm of those working on this off-line analysis almost delayed real data taking, which began at sunset.

And like the sun going round the earth, this cycle was repeated for four months. 


\section{ANALYSIS}

\section{A. Least-Square Fit}

From Eq. III-I the quantity $A$ we are attempting to determine is the slope of a linear relation between the cross section and target polarization. The technique used to extract $A$ is to do a least squares., fit of our data which were essentially cross sections (numbers of counts/ monitor) and target polarizations, to a straight line intercept $\sigma_{0}$ and slope of $\sigma_{0} \mathrm{AH}_{f}$. This yields $\mathrm{AH}_{f}$, which, when combined with our knowledge of $\mathrm{H}_{f}$ discussed in the next section, gives the desired value of $A$. The error is the error in the fit and includes the statistical error from the number of counts as well as the error due to uncertainty in the beam monitor. Possible systematic and other random errors are discussed below. The solution of this least-sqaure fit appears in many places 40 but some comments are appropriate since our problem is different in some respects from the one quoted in the reference. Due to the extremely small size of the expected asymmetry, the lack of kinematic separation of hydrogen and non-hydrogen scattering and the equal time devoted to each sign of polarization, there is a considerable simplification in the solution. The error due to the monitor requires separate treatment since it is not a simple counter whose error follows Poisson statistics. Drifts as well as noise contribute to fluctuations which we estimate to be $\delta M=1 \%$ per subrun (10 seconds) on the basis of comparison between the monitors and also calibration circuitry on the toroids.

The results are: 
$-80-$

$$
\begin{aligned}
& A=\frac{\sum_{i=1}^{N S U B} N_{i} P_{i}-\sum_{i=1}^{\text {NUB }} N_{i}\langle P\rangle}{H_{f}\left\langle Q^{2}\right\rangle \sum_{i=1}^{\text {SUD }} N_{i}} \\
& E_{\text {rror }} \text { (counters) }=\left(H_{f}^{2}\left\langle Q^{2}\right\rangle \sum_{i=1}^{\text {NUB }} N_{i}\right)^{-1 / 2} \\
& E_{\text {rror (monitors) }}=\frac{\left\langle P^{2}\right\rangle^{1 / 2} \delta M}{H_{f}\left\langle Q^{2}\right\rangle(N S \cup B)^{1 / 2}}
\end{aligned}
$$

$(\nabla I I-1)$

where $P_{i}, N_{i}$, and $M_{i}$ are the target polarization, number of events and number of beam particles in the $i$ th subrun. NSUB is the total number of subruns considered.

$$
\begin{aligned}
& \langle P\rangle=\sum_{i} P_{i} M_{i} / \sum_{i} M_{i} \quad \text { the arèreqge polarization } \\
& \left\langle\mathrm{P}^{2}\right\rangle=\sum_{i .} \mathrm{P}_{i}^{2} \mathrm{M}_{i} / \sum_{i} \mathrm{M}_{i} \quad \text { the mean-square polarization } \\
& \left\langle D^{2}\right\rangle=\left\langle\mathrm{P}^{2}\right\rangle-\langle\mathrm{P}\rangle^{2} \quad \text { the displacement from the mean }
\end{aligned}
$$
are independent.

$$
\text { Error }=\left(E^{2} \text { (COUNTERs) }+E^{2} \text { (monitors) }\right)^{1 / 2}
$$

(VI II)

$$
\text { Typically in a subrun: } \begin{aligned}
P_{i .} & =+22 \% \\
N_{i} & =250 \text { counts/missing-mass bin } \\
M_{i} & =2 \times 10^{12} \text { electrons } \\
o M & =0.01
\end{aligned}
$$


$-81-$

The averages for one of our running conditions were:

$$
\begin{aligned}
P & \approx 0.1 \% \\
P^{2} & \approx(0.22)^{2} \\
\text { NSUB } & \approx 15,000
\end{aligned}
$$

Thus the monitor error is about $15 \%$ of the magnitude of the counter errors and when added in quadrature changes the total statistical error by only a couple of percent of itself.

Equation VII-I can be further simplified to the form of III-2 if. half the subruns have polarization $\pm P$ and each subrun has the same amount of beam.

$$
A=\frac{P\left(\sum_{t} N_{i}-\sum N_{i}\right)}{H_{f} P^{2}\left(\sum N_{i}+\sum N_{i}\right)}=\frac{1}{P H_{f}} \frac{\sum_{ \pm} N_{i}-\sum N_{i}}{\sum N_{i}+\sum N_{i}} \quad(\nabla I I-3)
$$




\section{B. Test Asymmetries}

In making such a sensitive test (a few hundredths of a percent in $\epsilon)$ we cannot ignore the possibilities of random errors. To evaluate them we calculate asymmetries from our data which should be zero, even if there were a $T$ violation. This is done by pretending, for the purpose of calculation, that the target polarization is different than it actually is. Random gyrations of the data would result in non-zero values of these "test" asymmetries. To relate the "test" asymmetry values to a possible random error in our real asymmetry requires an assumption on the nature of possible random fluctuations. If the fluctuations are random, uncorrelated with anything, then each "test asymmetry f'ormed, no matter what sections of the dalta are called "positive" and which "negative" polarization (as long as their was no correlation with actual reversals) should give similar values within counting statistics. The real asymmetry should also have this same shiftt. (I'his systematic shift will of course fluctuate due to statistical errors.) We can weaken our assumptions on the nature of the errors. Let the actual target polarization reverse at a frequency $f$. We can assume that the fourier component of the fluctuations with frequency $f$ is the same as that with $\beta f$ where $\beta \approx 1$. Then a measurement of test asymmetries at target reversal frequencies $\beta f$ will give information on the random error in the real asymmetry. In practice we take values of $\beta$ of $1 / 2$, 2, 3 as well as other patterns which have phase lags of various number of degrees or patterns not of a single frequency. All of these patterns include equal amounts of data of both actual signs of polarization so 
that even if the cross sections were different, this would not effect the test asymmetries. Our data-taking pattern of 12 subruns for each sign of polarization and 2 complete cycles of + - polarization (a foursum) was designed to enable us to devise many test asymmetry patterns of higher and lower reversal frequency than the actual reversal frequency. Figure 15 shows these patterns. Most of them use three subruns as their basic unit of fake polarization reversal. They are all statistically independent of one another so that each one provides new information not contained in the others. Appendix D gives the criteria for statistical independence of these patterns.

What kinds of analysis can be made from test asymmetries to derive estimates of our random error?

(a) On-line, in real time: Five test asymmetries were displayed on the computer oscilloscope, each as a function of missingmass (12 bins), along with the $x^{2}$ with respect to 0 and with respect to the mean, the mean and the statistical error of the mean forleach pettern. These were watched almost constantly for significant deviations from their expected values of $(12,11,0 \pm 2$ s.d.) and data taking was stopped by the experimenter on the rare occasions when his subjective judgment indicated the situation was deteriorating and that we should withdraw. Most often this observation led to the discovery of the cause of the difficulty, usually an erratic shift in beam position or intensity, and at the beginning, computer program bugs. Sometimes, however, no "causc" was discovered, which simply meant something strange was going on 


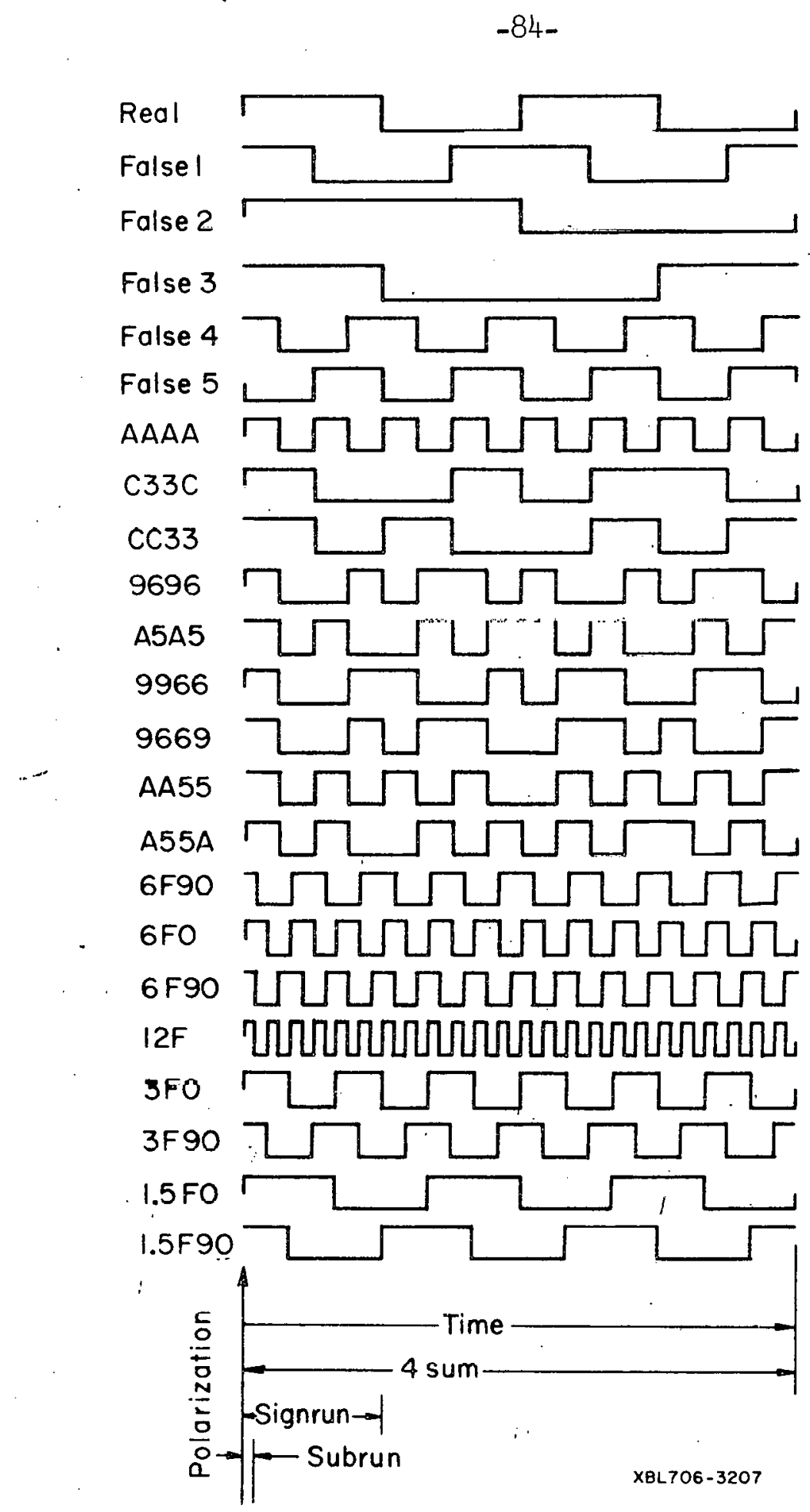

Fig. 15. Test asymmetry patterns. The names at left are nicknames used only by fritends. The first 5; False 1 through False 5 were observed on-line. 
$-85-$

that our monitoring devices could not detect (such as a failure of a scaler to be read or record correctly in a just barely significant digit). Throwing out data on the subjective test asymmetry criteria could not bias our real asymmetry results since, as mentioned above, the test asymmetries were statistically independent of the real asymmetry:

(b) Off-line analysis: Here, of course a much more quantitative analysis is needed to determine whether the test asymmetry values for all of the data included in.the analysis were consistent with no random error and, if not, what is the size of the random error. Several overlapping approaches were used, not because they were completely independent (they were not) but because they emphasized different aspects of any fluctuations.

Many of these techniques involved making a distribution curve of the number of occurrences of values of (asymmetry)/(statistical error) = number of standard deviations from zero. If there were only statistical errors (Eq. VII-2), this distribution would be a gaussian, centered at zero and of width 1 ( 1 standard deviation). Any statistically significant deviation from this is evidence for a non-statistics contribution to the error.

(1) Foursum by foursum distribution: A distribution is formed with entries of asymmetry/error taken from each missing-mass bin for each foursum separately. Thus there are 12 (number of mass bins) $x$ No. of foursumis (a few hurdred) $x$ No. of test asymmetry patterns included. This tests whether the data are 
uniformly good over time (foursums) and for each hodoscope element (missing-mass bin). It is possible to devise criteria of data rejection based on throwing out all foursums with entries greater then N standard deviations. Figure 16 shows some truly typical examples. Clearly the distributions are almost perfect gaussians.

(2) Mass-bin-asymmetry distribution: The "test" asymmetries are comblned for all the data at specfied running conditions (inciaent energy, spectrometer angle) just as the rcal asymmetry is. This results in 27 "test" asymmetry plots of asymmetry vs. missing mass for each of our running conditions. Some of these are shown in Fig. l.'. As before, $x_{\text {mean, }}^{2} x_{0}^{2}$, the mean and error of mean are calculated and, together with a skilled eye, serve as a basis for a subjective test as to wheller lle values are consistent with zcro. More quantitatively, each mass bin of each "test" asymmetry becomes an entry into a distribution of asymmetry/error values with 500-1000 entries. Figure 18 shows one these distributione. Again they are completely consistent with a gaussian centered at $O$ with 1 standard deviation width, indicat1ng no significant nonstatistics error. This distribution, especially the tails, is a direct test of what the fluctuations of the real asymmetry plot (which is our final results) might be. The absence of nonstatistics tails is strong evidence that large random fluctuations do not occur in the real asymmetries. 


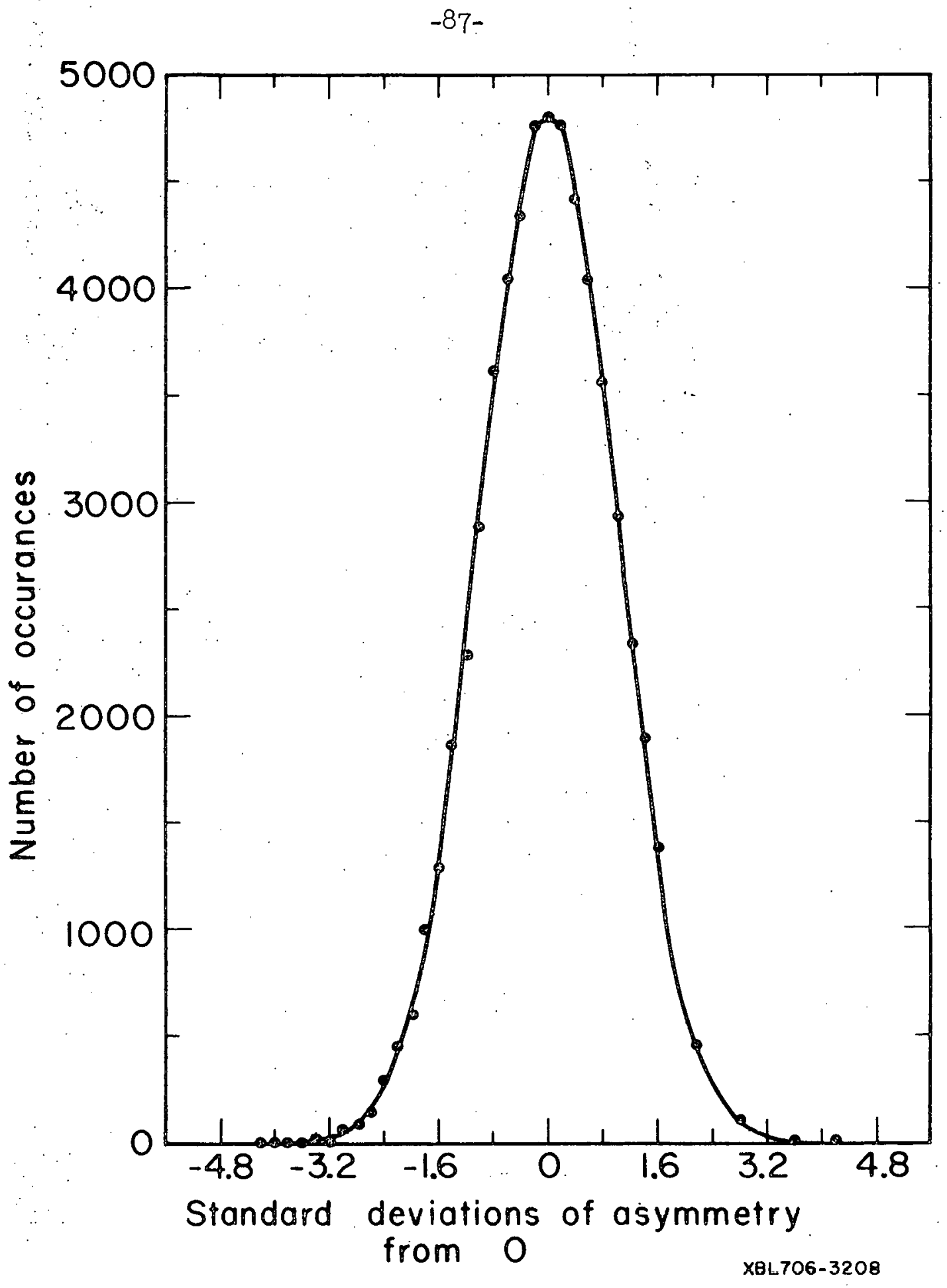

Fig. 16. Foursum by foursum distribution. Mean $=4.4 \times 10^{-3}$, width $=0.9995$ S.D., $68.1 \%$ of events < 1 S.D. from 0. $95.41 \%$ of events <2 S.D. from $0.99 .74 \%$ of events <3 S.D. from 0 and $100 \%$ of events $<4$ S.D. from 0 . 


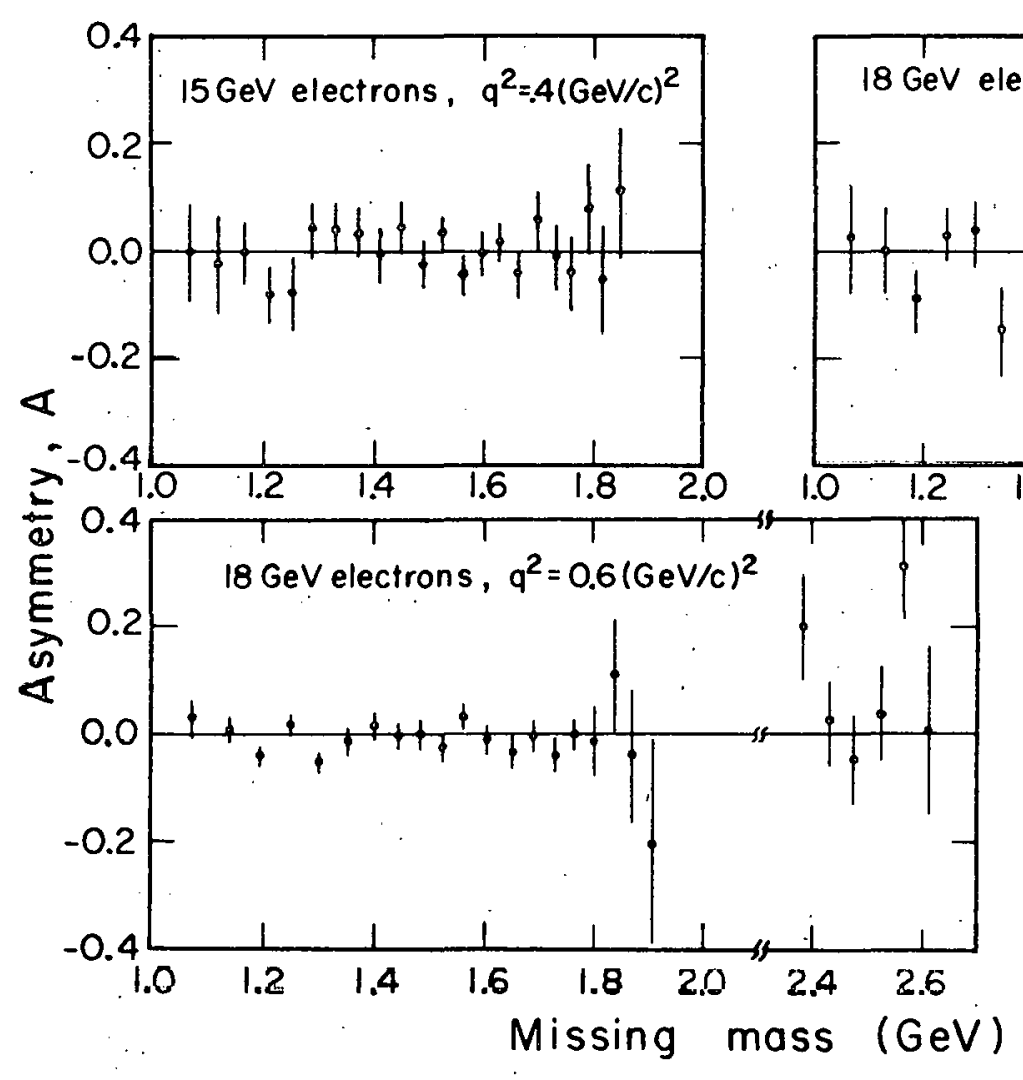

Fig. 17. Test asymmetries as a function of missing mass (False 1 ). $q^{2 \approx .4}$ electrons, $x_{\sigma}^{2}=14.5, x_{\text {mean }}^{2}=14.4$, Average $=(-.17 \pm .87) \%$; $q^{2 \approx 1} .0$ electons, $x_{0}^{2}=22.4, x_{\text {mean }}^{2}=22.3$, Average $=(-.38 \pm 1.22) \%$; $q^{2 \approx .6}$ electrons, $x_{0}^{2}=16.3, x_{\text {mean }}^{2}=15.3$, Average $=(.55 \pm .58) \%$; $q^{2 \approx .4}$ positrons, $x_{0}^{2}=13.6, x_{\text {mean }}^{2}=13.6$, Average $=(-.26 \pm 1.41) \%$. 


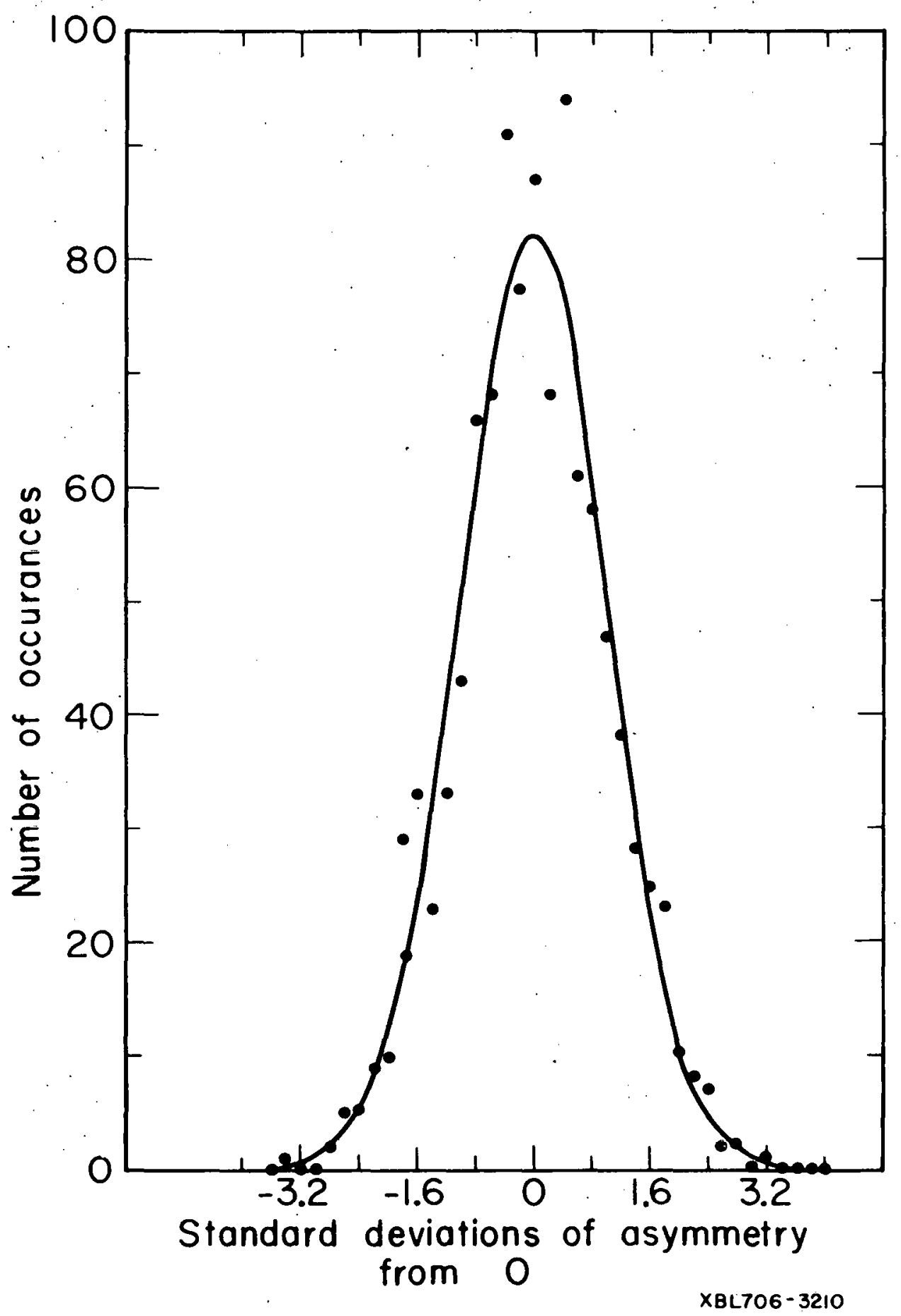

Fi.g. 18. Distribution of test asymmetries. All $q^{2 \approx .6 . ~ t e s t ~}$ asymmetries, 27 spectra of asymmetry versus missing mass with 39 mass bins for a total of 1053 entries. Mean $=-2.2 \times 10^{-2}$ S.D., width $=1.023$ S.D., $67.8 \%$ of events $<1$ S.D. t'rom U, $95.1 \%<2$ S.D. from $0,99.0 \%$ $\leq 3$ S.D. from $0,100 \% \leq 4$ S.D. from 0 . 
(3) Spectrum Asymmetry distribution: Each of the $27 \times 4$ (No. of running conditions) plots mentioned in (2) has a mean asymmetry and error. A distribution of asymmetry/error of these quantities is shown in Fig. 19. As in all cases above, this is completely consistent with the expected gaussian. This distribution is valuable in determining any biases or offsets of an ent1re spectrum of missing mass such as might be caused by a defective monitor. Since each entry has a tiny statistical error corresponding to about a quarter of our total data (over 100 million events for our four running conditions) we are very sensitive here to very slight fluctuations, not even significant for cach individual missing-mass bin. (e.g. a 2 S.D. shift of the mean corresponds to a 0.4 S.D. shift per bin for 25 missing-mass bins.)

(4) To get an even more quarlitative estimate or lite lotal error (systematic plus"statistical plus anything-else error), the "test" asymmetries themselves were used to calculate the error. The difference between a "test" asymmetry value and zero is duc to this total error and the appropriate average of all 27 "test" asymmetries is an estimate of the total error. We form:

$$
X_{0}^{2}=\sum_{i=1}^{27}\left[\frac{\text { Asymmetry }}{\text { ERROR }_{i}}\right]^{2}
$$




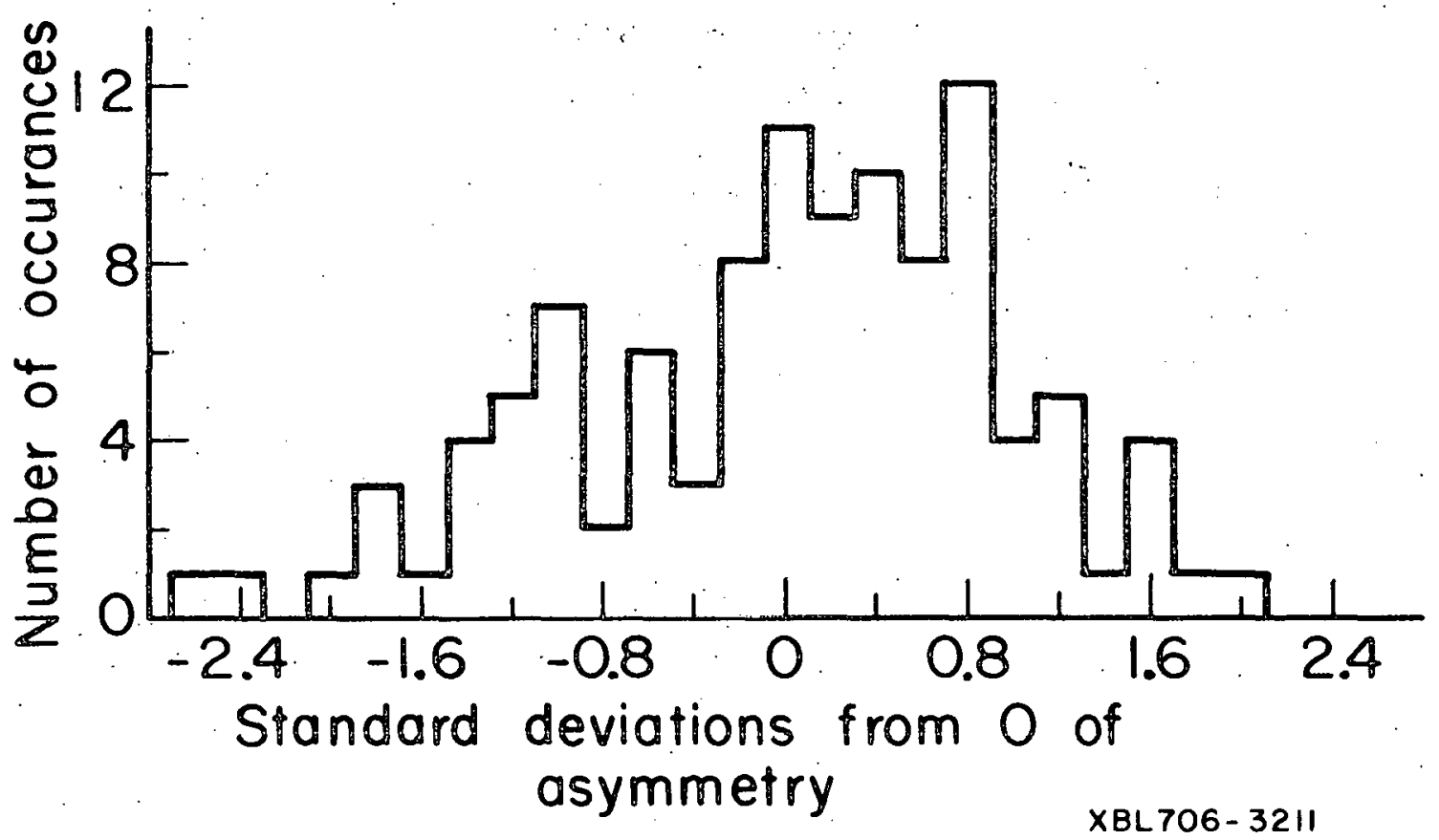

Fig. 19. Distribution of average asymmetries from each of the 108 spectra of test asymmetries.

Mean $=0.01$ S.D., width $=0.91$ S.D., $69 \%$ of events $<1$ S.D. from $0,97 \% \leq 2$ S.D. from $0,100 \% \leq 3$ S.D. from 0 . 
where the sum is over all "test" asymmetry patterns and the asymmetry is that for one of the missing-mass bins. Noting that the error is about the same for each pattern (since the same number of events are involved and all come from the same data) and also that $\chi_{0}^{2}$ should equal the number of degrees of freedom (27) if the error is really the total error we have:

$$
\text { TOTAL ERROR }=\left[\frac{\sum_{i=1}^{27} A_{i}^{2}}{27}\right]^{1 / 2} \text {. }
$$

Thus the appropriate average mentioned above is the root-meansquare. If there are no random fluctuations the total error should equal the statistics error. Figure 20 shows the total error as calculated above as well as showing the countingstatistics error as the bars. The average ratio of (total error)/(courlting-statistics error) is also shown and is very close to 1.00 .

This "test" asymmetry ananlysis gives us confidence that any random nonstatistical error is negligible and our true errors are accurately represented by the couriting-statistics errors. 


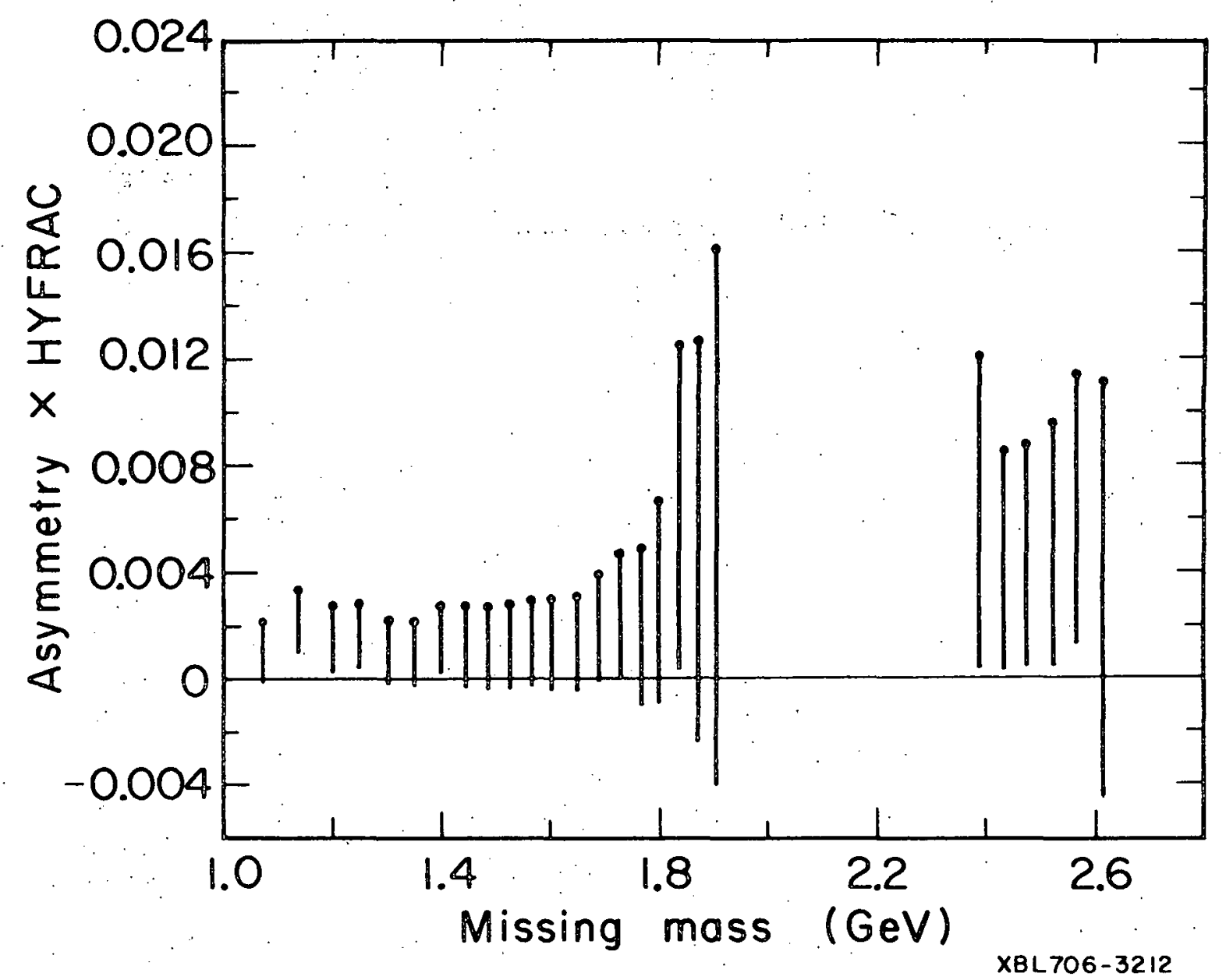

Fig. 20. Root mean square (total) error for $q^{2 \approx 0}$.6. The error bars are the counting statistics errors. From the graph Total error $=0.96 \mathrm{x}$ (the statistical error) when averaged. 


\section{Deadtime}

All of the above information on the "test" asymmetry results includes a small correction due to the deadtime in our detection system. This correction has almost no effect on the real asymmetry (usually less then 0.I S.D. but with a maximum of 0.4 S.D.). The necessity for this correction was discovered through analysis of the "test" asymmetries and in particular false 5. Cormarison of counts/monitor versus beam intensity done on the actual data of the experiment shuwed a deadtime of 60 no arter each event (or $6 \%$ at $1-1 / 2$ events per $1.5 \mu$ sec pulse). While we do not understand why we have such a large deadtime, its existence throughout our data taking is indisputable as shown in the plot of Fig. 2l. A deadtime correction is of course rate dependent since the more events the more deadtime to recover from them. If this deadtime is combined with a fluctuating beam intensity a non-zero asymmetry can be generated.

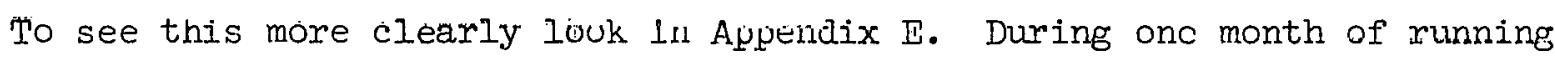
there was a $3 \%$ fluctuation in the beam intensity which followed the same pattern as false 5 resulting in a 5 S.D. shift from zero. This corresponded to an increāsing beam irtensily fıü the time the bcam was turned on after a polarization sign change to when it was turned off to change sign again. Other, much smaller fiuctuations in beam intensity were found to follow a couple of other 'test" asynmetry patterns and our deadtime correction eliminated small deviations from true gaussian distributions that had troubled us. No significant alteration of the real asymmetry was necessary since there was fortunately little correlation between beam intensity and actual target polarization reversals. 
$-95-$

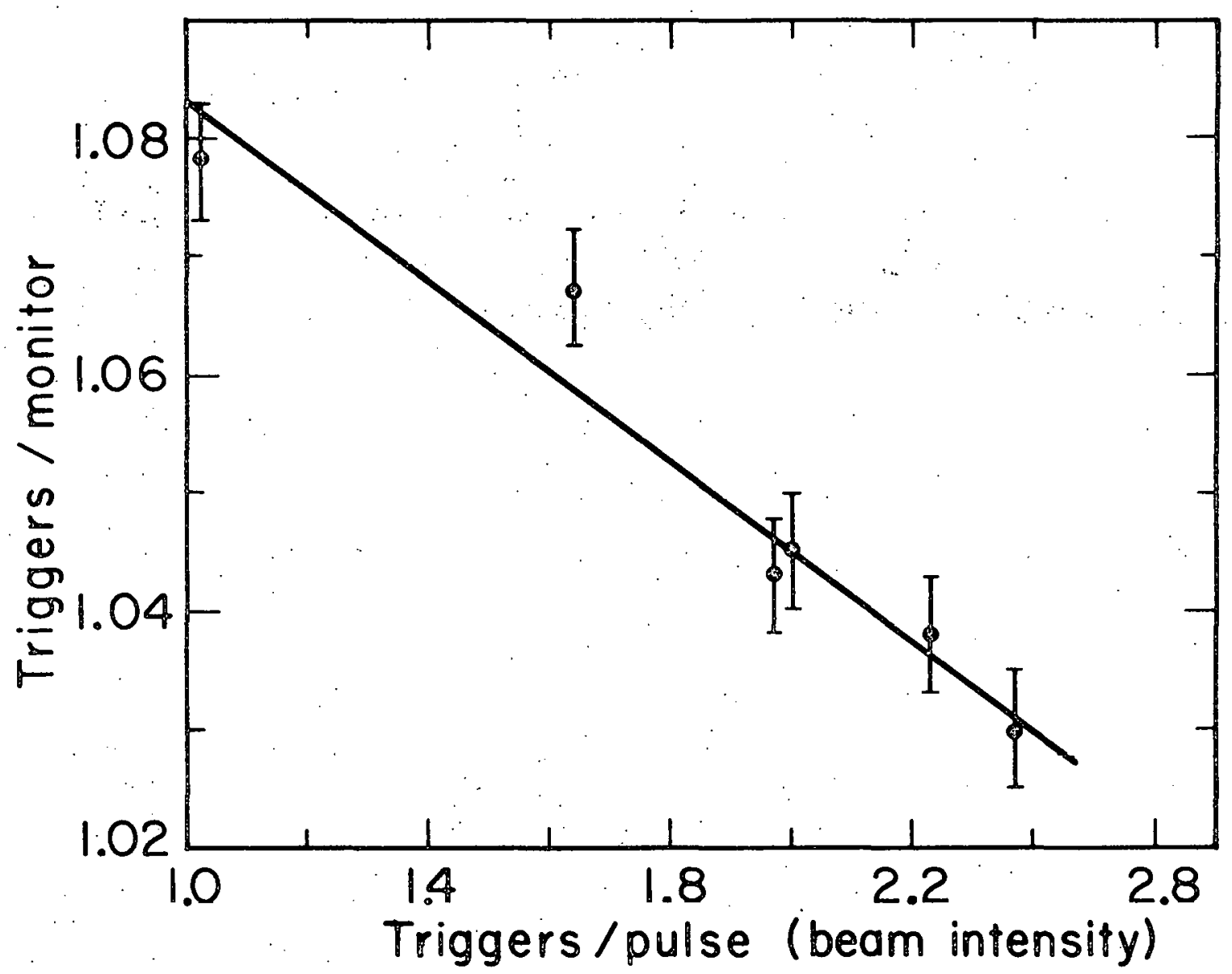

XBL706-3213

Fig. 21. Deadtime. Points represent consecutive runs at different beam intensity. The slope of the line is $4 \%$ change in triggers/monitor for each increase of 1 trigger/pulse. 


\section{Monitors}

Another quantity which should be zero is the asymmetry of one beam monitor with respect to another. The asymmetry is defined as usual as $t$ he relative difference of monitor $\mathrm{M}^{2}$ normalized to monitor $\mathrm{M}^{2}$ :

$$
A_{\text {mon }}=\frac{1}{P H_{f}} \frac{M_{+}^{\prime} / M_{+}^{2}-M_{-}^{\prime} / M_{-}^{2}}{M_{+}^{\prime} / M_{+}^{2}+M_{-}^{\prime} / M_{-}^{2}}
$$

where + and - represent the sign of the target polarization and the fortuitous factor $\mathrm{PH}_{\mathrm{f}}$ is included only for direct comparison with the asymmetries of the actual hodoscope counters.

The asymmetry of the number of counts with respect to monitor $\mathrm{M}^{1}$ (VII-3) should be the same as the asymmetry with respect to $\mathrm{M}^{2}$ if we can put any faith at all in our data. This difference can be expressed in terms of $A_{\text {mon }}$ by some arithmetic yiclding:

$$
A_{\text {mon }} \approx \frac{1}{P H_{+}}\left\{\left[\frac{N_{+} / M_{+}^{\prime}-N_{1} / M_{-}^{\prime}}{N_{+} / M_{+}^{\prime}+N_{-} / M_{-}^{\prime}}\right]-\left[\frac{N_{+} / M_{+}^{2}-N_{-} / M_{-}^{2}}{N_{+} / M_{+}^{2}+N_{-} / M_{-}^{2}}\right]\right\}
$$

in the approximation that $\mathrm{N}_{+} \approx \mathrm{N}_{-}$and $\mathrm{M}_{+}^{7}, 2=\mathrm{M}_{-}^{7}, 2$.

I'able IV shows $A_{\text {mon }}$ for the three monitors and four different running conditions and compares it with the appropriate statistics errors. The monitor asymmetry is always lese then half the statistics error on the combined data and less then $1 / 10$ the error for each mass bin and thus makes little or no contribution to the uncertainty in our results. The monitor asymmetries are consistent with zero when their errors are taken 


\section{TABLE IV. MONITORS}

How much would results change if different monitor used?

\begin{tabular}{|c|c|c|c|c|c|c|c|c|}
\hline \multirow[b]{2}{*}{$E_{0}$} & \multirow[b]{2}{*}{$q^{2}$} & \multirow[b]{2}{*}{$e^{ \pm}$} & \multicolumn{2}{|c|}{$\begin{array}{c}\text { Counting statistics } \\
\text { errors }\end{array}$} & \multicolumn{4}{|c|}{$\begin{array}{c}\Delta \text { due to different } \\
\text { monitor }\end{array}$} \\
\hline & & & All bins & One bin & Mon & B & Mon & $\ddot{C}$ \\
\hline 18 & 0.6 & - & $\pm 0.06 \%$ & $\pm 0.24 \%$ & 0.007 & & 0.000 & \\
\hline 18 & 1.0 & - & $\pm 0.16 \%$ & $\pm 0.7 \%$ & 0.01 & $\%$ & 0.09 & $\%$ \\
\hline 15 & 0.4 & - & $\pm 0.11 \%$ & $\pm 0.4 \%$ & 0.05 & $\%$ & 0.05 & $\%$ \\
\hline 12 & 0.4 & + & $\pm 0.12 \%$ & $\pm 0.35 \%$ & 0.06 & $\%$ & 0.07 & $\%$ \\
\hline
\end{tabular}


$-98$

into account. The monitor asymmetries serve as a check on the estimated size of our monitor errors. These errors have already been included in our total error as shown in Section $A$ and hence we do not add on these monitor asymmetries as additional errors. 
$-99-$

F. Regions

The necessity for dividing the data into the three kinematic regions of the target enabled us to make a check on the absence of systematic error. There is one particular type of error which we were worried about since it was the only one which could be physically correlated with target polarization. This is the liquid helium level discussed in Section V.D. Any systematic lowering of the helium level during one sign of polarization would result in the top target region having a noticeable different asymmetry than the others, especially the bottom region. On one day we were actually able to detect such a difference which was accompanied by uneven cryostat operation. A small hole in the can was found to be the culprit. Aside from this (the data was thrown out) there was no statistically significant difference between asymmetries in the three target regions. 


\section{F. Selection of Data}

Not all the data that was recorded on magnetic tape was of uniform quality as regard to suscetibility to systematic and random error. Each foursum, as a complete independent unit of data, was either accepted or rejected from further analysis on the basis of several consistency requirements. Amorig these were:

(1) Beam stability: was the beam intensity constant to better then $x \%$ (usually 40\%) from subrun to subrun within the foursum? See Fig. ?? for how this cut was made. It is typical of how the others were done.

(2) Monitor agreement: did the three independent beam intensity monitors agree with each other to $x \%$ (usually $6 \%$ ) during each sulurus.

(3) Beam position: Di.d the beam position as monitored upstream of the sweeping magnets change by a significant fraction of its natural high frequency jitter from subrun to subrun. (After study, - no actual cut was made bascd on beam position.)

(4) Trigger consistency: ald the triggers/monitor vary by more then $x$ S.D. (usudily 4) from subrun to subrun.

(5) Hodoscope: did the events/monitor in each of the 10 hodoscope counters vary by more then $x$ S.D. 


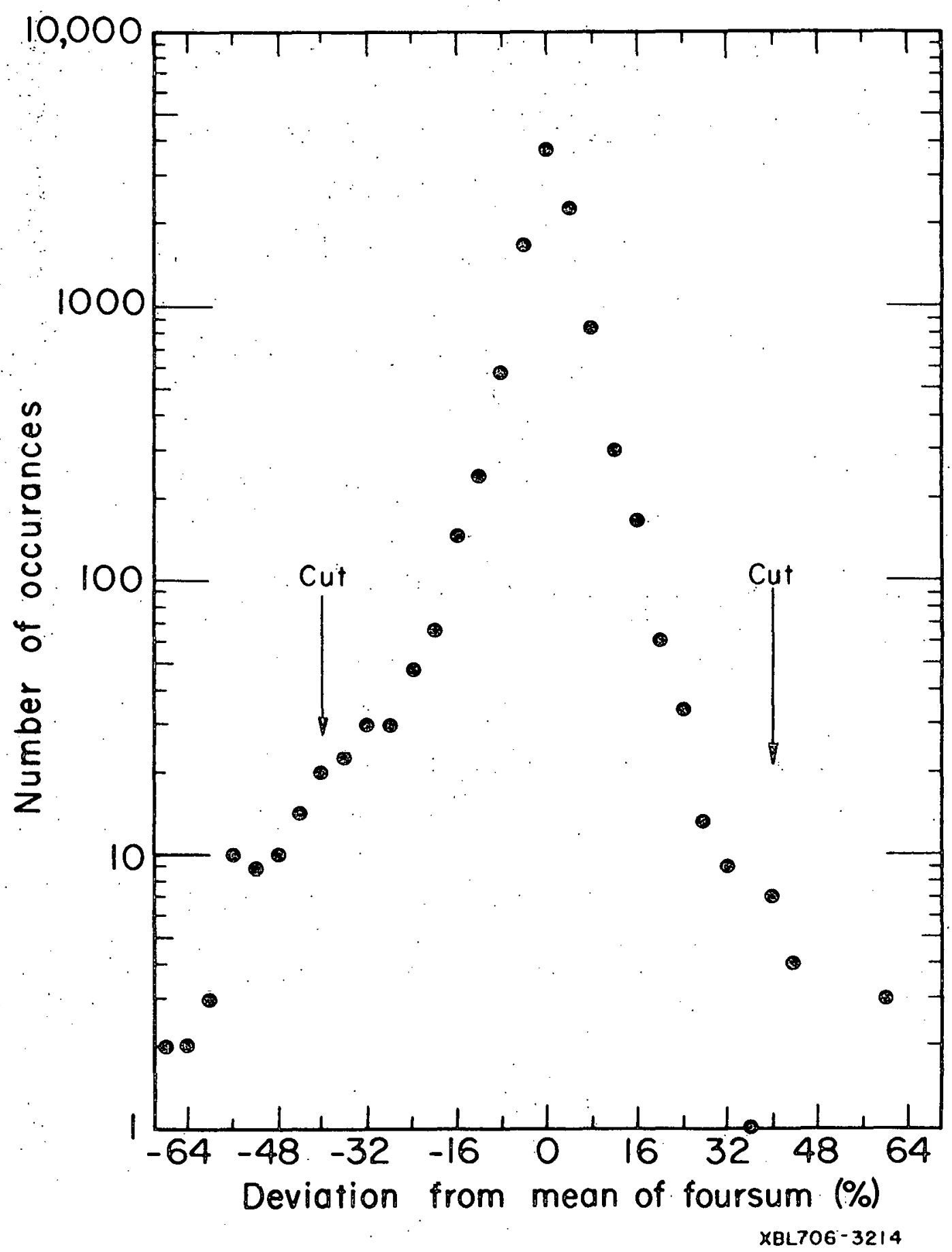

Fig. 22. Beam intensity with respect to mean of foursum. The data rejection cuts are shown. 
(usually 5) from subrun to subrun. This was a. test of whether a scaler had miscounted or been misread by the computer, and error which occurred 20 times out of $\geq 10$ million readings.

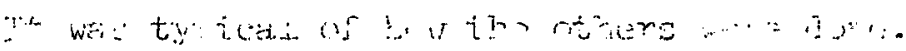

(6) Arcidental rate: Did the accidental rate change by more then. X S.D. (usually 4 S.D.) from subrun to subrun.

(7) Target polarization: Many checks to see if the polarization recorded is the sign and approximate magnitude expected.

(8) Test Asymmetrles: Was the $x^{2}$ with respect to 0 of each 'test' asymmetry less then some huge number (100 with 12 experted.). This was the last test, designed to catch any horirendous trouble that slipped through the other criteria.

Even perfectly good data will be rejected by these criteria due to expected statistical fluctuations of the variables and thus it may in principle be difficult to determine what "good" limits on these cuts should be. In practice we found that there were certain foursums which had to be thrown out due to troubles like misread scalers and widely differing beam monitors. If one formulated ad hoc "reasonable" (a t'ew percent loss due to statistical fluctuations) values of $\mathrm{x}$ the clearly bad foursums were thrown out in an unbiased way (each foursum had to meet the same criterla), only $15 \%$ of the total data was rejected, and the quality of the data assumed the pristine form described above. 


$$
-103-
$$

Further checks of the validity of the cuts were obtained by changing them to very strict (reject $50 \%$ of data) and very loose (reject $5 \%$ of data) and observing no statistically significant change in the values of any of the asymmetries, "test" or real. We believe the always-necessary operation of throwing out bad data was accomplished in a natural and unbiased manner. None of the cuts was correlated with a possible asymmetry. The final cuts resulted in a loss of about $15 \%$ of the data, making an extremely small increase in the statistical error. 


\section{HYDROGEN FRACTION}

Since it was impossible to separate the small fraction (10\%) of the scatters which occurred from free polarizable protons (the hydrogen in the target) from the background events from heavy nuclei, we had to make an independent measurement of the hydrogen/background ratio (called hydrogen fraction or Hyfrac $H_{f}$ ). We expected that $H_{f}$ would be a function of the missing mass of the outgoing hadronic state. For example, elasticaliy scattered electrons from f'ree protons should have a $\delta$ tunction tior missing mass (ignoring spreading due to experimental resolution and radiative corrections) while elastic scatters from bound nucleons which are not at rest due to Fermi motion within the nucleus (200 MeV/c momentum) should have a missing-mass distribution about $200 \mathrm{MeV}$ wide. Thus $\mathrm{H}_{\mathrm{f}}$ should be a maximum at the elastic scattering and a minimum between elastic and the one-pion threshold where no scattered electrons from free protons should appear (again ignoring resolution and radiative corrections). Since the resonances have widths comparable to the spread Que to Fermi motion we did not expect a large increase in $\mathrm{H}_{f}$ at the excited nucleon states.

Our determination of $\mathrm{H}_{f}$ consiatcd of finding the micsing-mass spectrum of hydrogen and carbon and then comparing them with the spectrum from the polarized target. We installed carbon or $\mathrm{CH}_{2}$ targets of the same radiation length as the butanol and used a $\mathrm{CH}_{2}-\mathrm{C}$ subtraction to get a hydrogen spectrum. A standard carbon (hydrogen) missing-mass spectrum was normalized to the amount of carbon (hydrogen) in a $\mathrm{CH}_{2}$ target of the same weight as our polarized target. A backeround of 
about $7 \%$ due to vacuum window, He bags, etc. was subtracted out. Thus a $\mathrm{CH}_{2}$ target spectrum could be fit by $\mathrm{A}_{\mathrm{h}} \times$ (standard hydrogen spectrum) $+A_{C} \times$ (standard carbon spectrum) where $A_{c} / A_{h}=1$. The butanol spectrum around the elastic peak (where there was the most structure and most $c$, $\mathrm{H}_{2}$ difference and hence the best fit) was fitted by a least square method to a linear ' combination of standard hydrogen and standard carbon spectrum. Typically $A_{c} / A_{h}=1.7$ which indicates that our polarized target, as seen by the beam, had less hydrogen then pure butanol $\left(\mathrm{C}_{4} \mathrm{H}_{9} \mathrm{OH} \approx \mathrm{CH}_{2}\right)$, as is expected. This is due to a background of carbon or carbon-like nuclei associated with the target such as aluminum can and vacuum windows and He bag $\left(0.2 \mathrm{gms} / \mathrm{cm}^{2}\right)$ teflon bags to hold the butanol $\left(0 .\left(25 \mathrm{gms} / \mathrm{cm}^{2}\right)\right.$ and liquid hellium in the can $\left(0.38 \mathrm{gms} / \mathrm{cm}^{2}\right)$ and unknown amounts of solder to seal the can up. The butanol-water target mixture was approximately $2 \mathrm{gms} / \mathrm{cm}^{2}$ : $A_{c} / A_{h}$ depended on the size of the sweep of the beam, being larger $(\sim 1.9)$ when the sweep was larger then the target and smaller ( 1.4) when the sweep was smaller than the target (missing the ends where the density of plastic bag and liquid helium was greatest). Thus the geometrical fuzziness of our target caused it to present a smaller fraction of free protons to the beam than a large block of $\mathrm{CH}_{2}$. We ran with a large beam sweep to irradiate uniformly the entire target so that our polarization readings would reflect the polarization of the target where the beam hit and we would not have to deal with a contribution to the polarization reading of an unused, highly polarized (no radiation damage) section of the target. To callculate $\mathrm{H}_{\mathrm{f}}$ for all values of missing mass, carbon, $\mathrm{CH}_{2}$, and 


\section{$-106-$}

target-empty data were taken at all values of the missing mass and a subtraction again determined the hydrogen spectrum $\left(\mathbb{N}_{h}\right)$ and carbon spectrum $\left(N_{c}\right)$. Then for the alcohol target $1 / H_{f}=$ (total counts) $/$ (hydrogen counts) $=\left(A_{h} N_{h}+A_{c} N_{c}\right) / A_{h} N_{h}=I+A_{c} / A_{h} \cdot N_{c} / N_{h}$ where $N_{c}$ and $N_{h}$ depend on the cross sections for $\mathrm{dC}$ and ep scattering. Figure 23 shows $\mathrm{H}_{f}$ as a function of missing mass for the four values of $\mathrm{q}^{2}$ used in the experiment. We estimate the uncertainty of these values to be about $20 \%$, based on repeated measurements on different days and a comparison with another method described below. The uncertainty is due to (a) lack of absolute calibration of the monitor (it changed from day to day) and (b) different targets on different days with varying amounts of alcohol, bag, liquid helium and solder. Since $\mathrm{H}_{f}$ is only a normalization factur, and since we are looking for the significance of the deviation of our asymmetry from zero (number of standard deviations) an uncertainty in $\mathrm{H}_{\mathrm{f}}$ does not. alter the significance of our results.

An alternate method, using only a carbon target, was used to check the accuracy of the complex fitting procedure described above. Missingmass spectra from an alcohol target and a carbon target (same number of radiation lengths) were normalized to the low-missing-mass ( $<850 \mathrm{MeV}$ ) tails of the spectra where none of the counts are due to free protons, which cut off at the nucleon mass. (Radiative effects increase the missing mass.) The difference between the two spectra is due to the free protons in the butanol target. A typical set of spectra are shown in Fig. 24. The two methods agree to better than $20 \%$. 


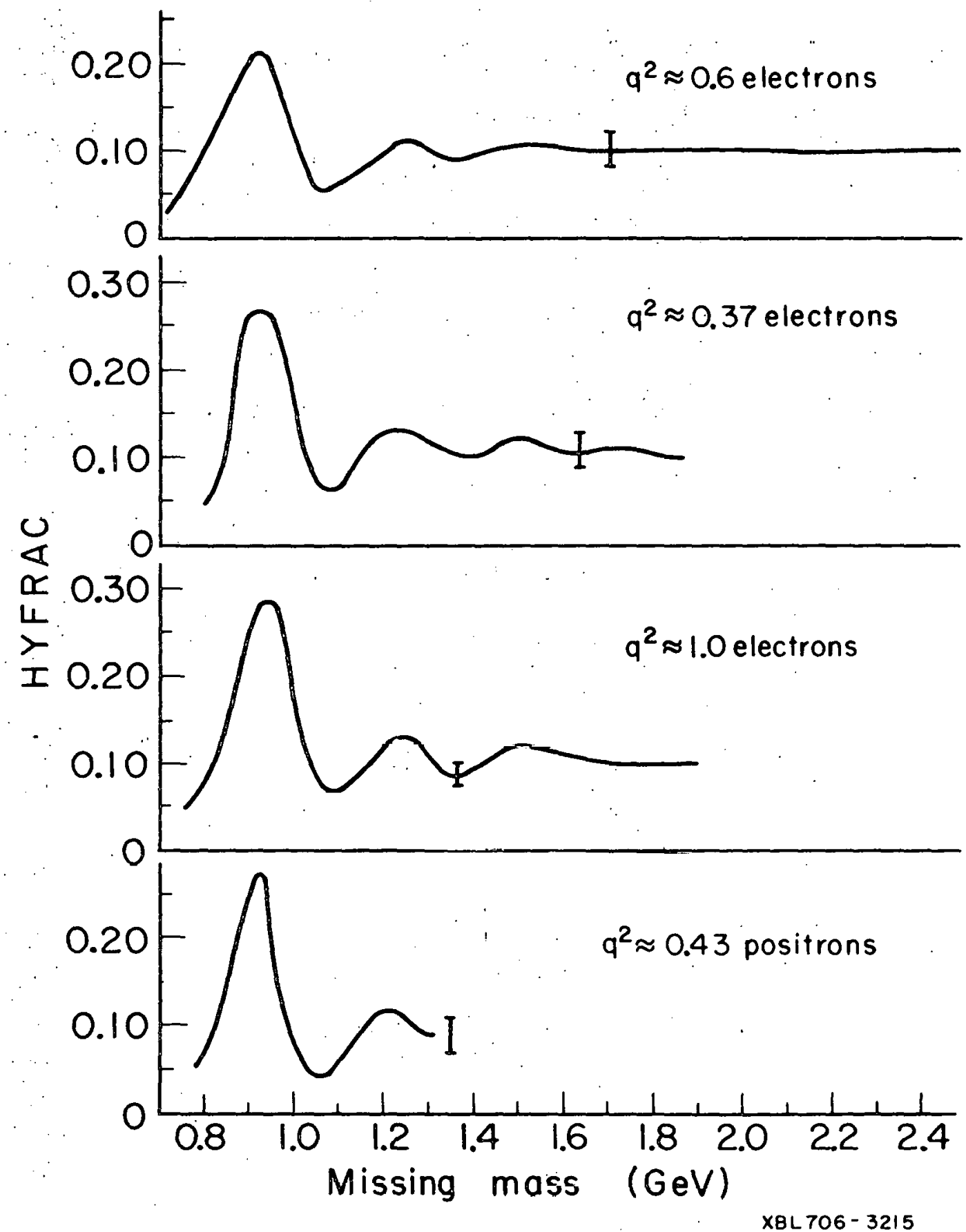

Fig. 23. Hydrogen Fraction as a function of missing mass for our four running conditiono. 

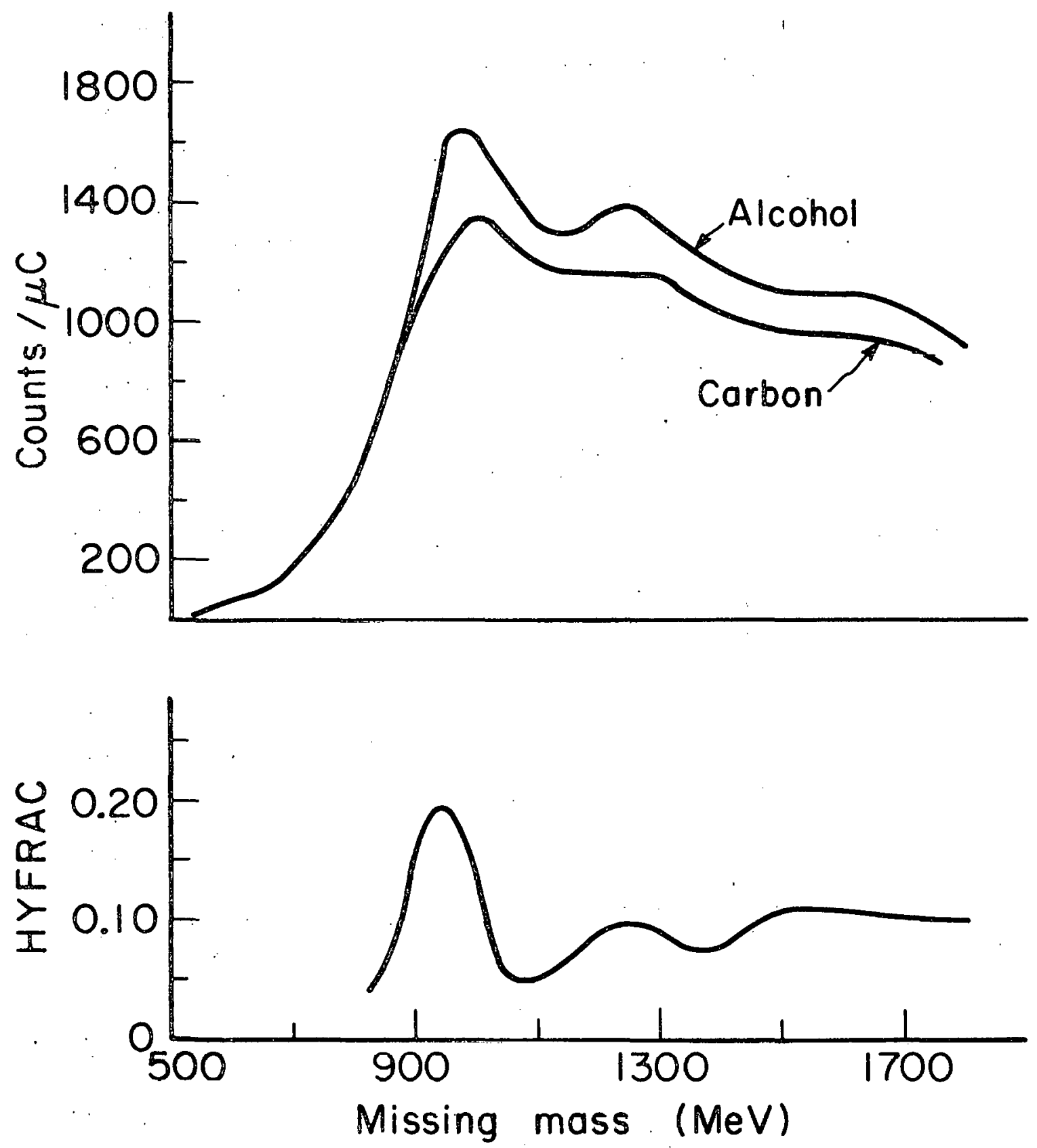

XBL706-3216

Fig. 24. Butal Alcohol target and carbon missing-mass spectra (courlis/Coulomb of beam), normalized to the tails bclow the elastic peak. Below is the hydrogen fraction calculated from these spectra. $q^{2} \approx 0.6$. 
IX. RESULTS

The final results of asymmetry $A$ as a function of missing mass for the four different running conditions (scattering angle and incident energy) are show in Fig. 25. Tables V, VI, VII, and VIII' show these same results. Table IX shows the results averaged over the electroproduced resonances. The results of chen and al. are shown for comparison at the bottom of the Table $\mathbb{D X}$. The resonance regions are important because there one partial wave dominates to some extent over the many others present.

There is no statistically significant deviation from zero asymmetry. (See Appendix $F$ on the apparent asymmetry at $q^{2}=0.6$, missing mass = $1200 \mathrm{MeV}$.$) Thus we have found no evidence for a time-reversal-$ invarience, violation in this reaction. Our errors rule out an asymmetry greater than 3 to $4 \%$ at a 2 S.D. (95\% confidence level) which is far below the $30 \%$ predicted by the naive model mentioned in section II. It is extremely unlikely that a T-violation and a $2-\gamma$-exchange asymmetry would cancel each other over the wide range of kinematics in our data (especially since $2-\gamma$ exchange is expected to be very small). The absence of asymmetry in the positron data taken at slightly different kinematic conditions is a confirmation that no cancellation occurs (they would then add for the positron data).

Unfortunately it is now apparent that this simple model is not necessarily adequate to describe the complex physical situation. Complications are:

(1) A glance at the cross sections, Fig. 24, shows that the reso- 

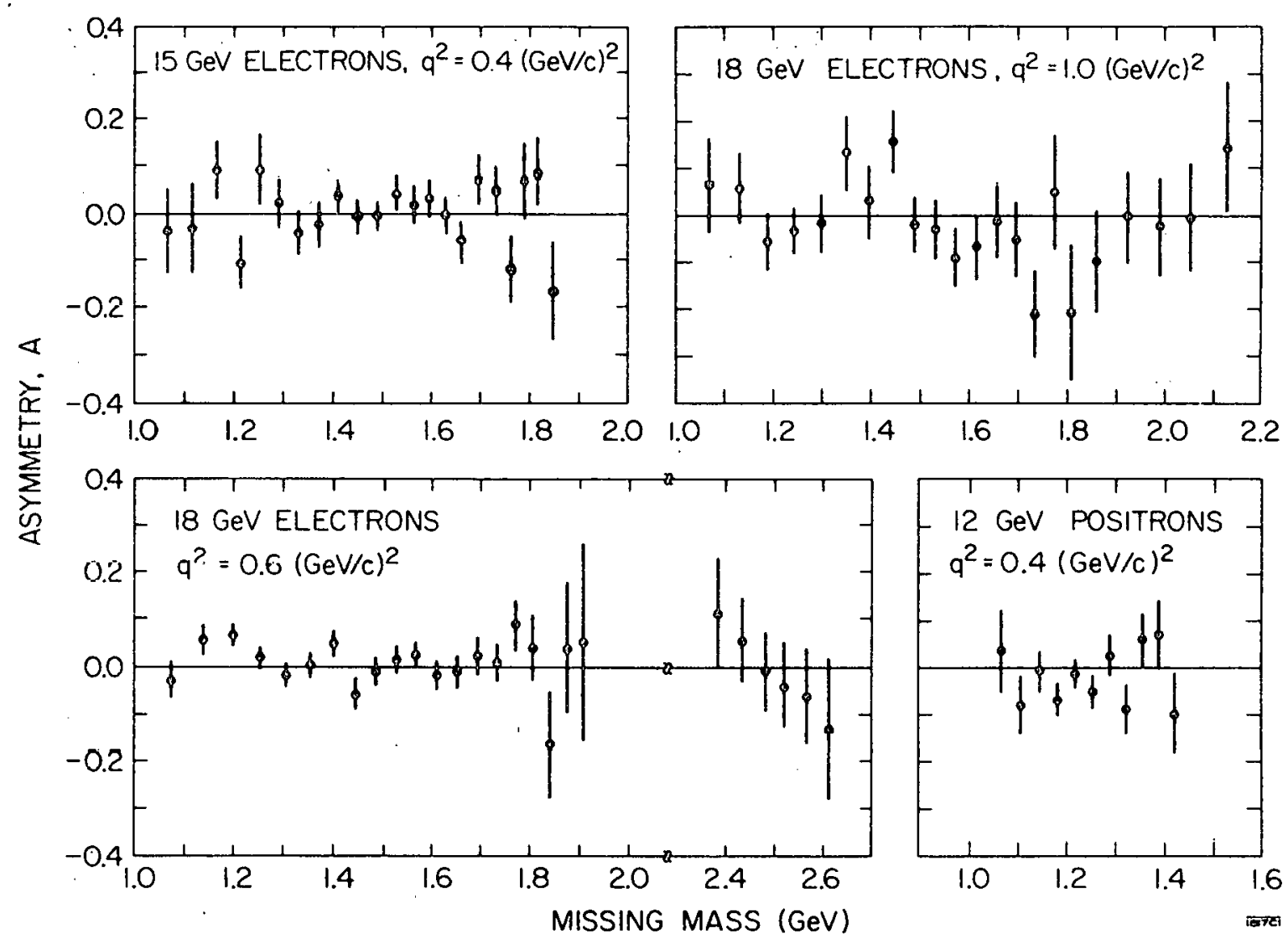

XBL $708-1712$

Fig. 25. Results. Asymmetry versus missing mass for our four ruming conditions. The resolution is $1-1 / 2$ mass bins. 
$-111-$

Table V. Asymmetry $A$ in ep $\rightarrow$ e $\Gamma$ inelastic scattering. The error $\triangle A$ is the statistical error.

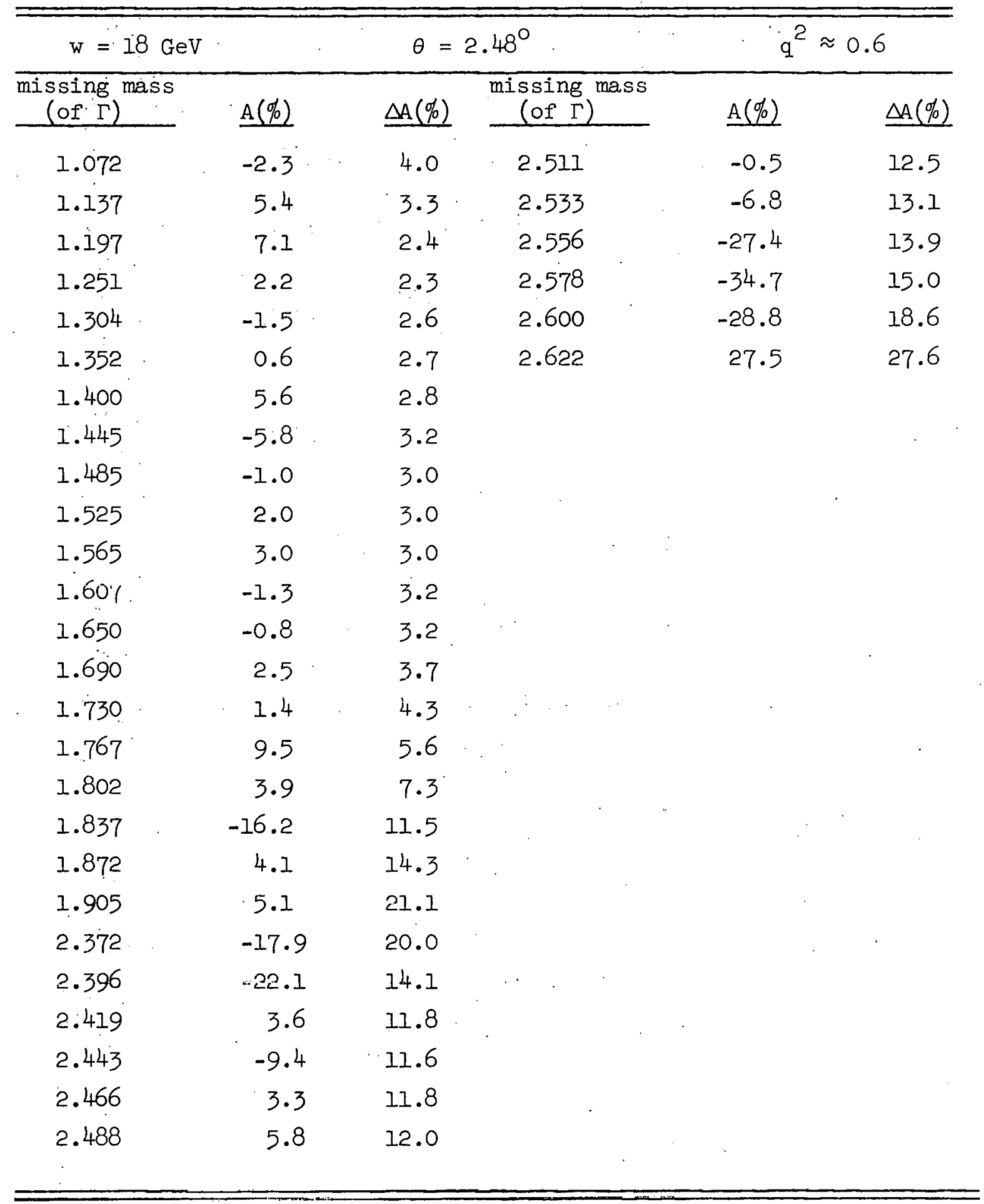


Table VI. Asymmetry $A$ in $\mathrm{ep} \rightarrow \mathrm{e} \Gamma$ inelastic scattering. The error $\triangle \mathrm{A}$ is the statistical error.

\begin{tabular}{|c|c|c|}
\hline $\mathrm{w}=15 \mathrm{GeV}$ & $\theta=2.37^{\circ}$ & $q^{2} \approx 0.4$ \\
\hline missing mass (of $\Gamma$ ) & $A(\%)$ & $\triangle A(\%)$ \\
\hline 1.065 & -4.0 & 8.9 \\
\hline 1.115 & -3.0 & -9.3 \\
\hline 1.165 & 9.9 & 6.2 \\
\hline 1.212 & -10.0 & 5.5 \\
\hline 1.252 & $9.3-$ & 7.0 \\
\hline 1.290 & 2.2 & 5.3 \\
\hline 1.330 & -4.4 & 4.7 \\
\hline 1.370 & -6.1 & 4.7 \\
\hline 1.410 & 5.0 & 4.8 \\
\hline 1.450 & -0.7 & 4.7 \\
\hline 1.488 & -0.1 & 4.1 \\
\hline 1.526 & 4.2 & 3.7 \\
\hline 1.561 & 0.2 & 3.8 \\
\hline 1.594 & 3.0 & 4.0 \\
\hline 1.627 & -0.8 & 4.5 \\
\hline 1.660 & -6.2 & 4.9 \\
\hline 1.695 & $7 \cdot 7$ & 5.3 \\
\hline 1.730 & 4.7 & 6.5 \\
\hline 1.760 & -12.1 & 7.4 \\
\hline 1.787 & 8.3 & 9.3 \\
\hline 1.815 & 9.2 & 12.0 \\
\hline 1.845 & -18.1 & 16.6 \\
\hline
\end{tabular}


Table VII. Asymmetry $A$ in ep $\rightarrow$ e $\Gamma$ inelastic scattering. The error $\triangle A$ is the statistical error.

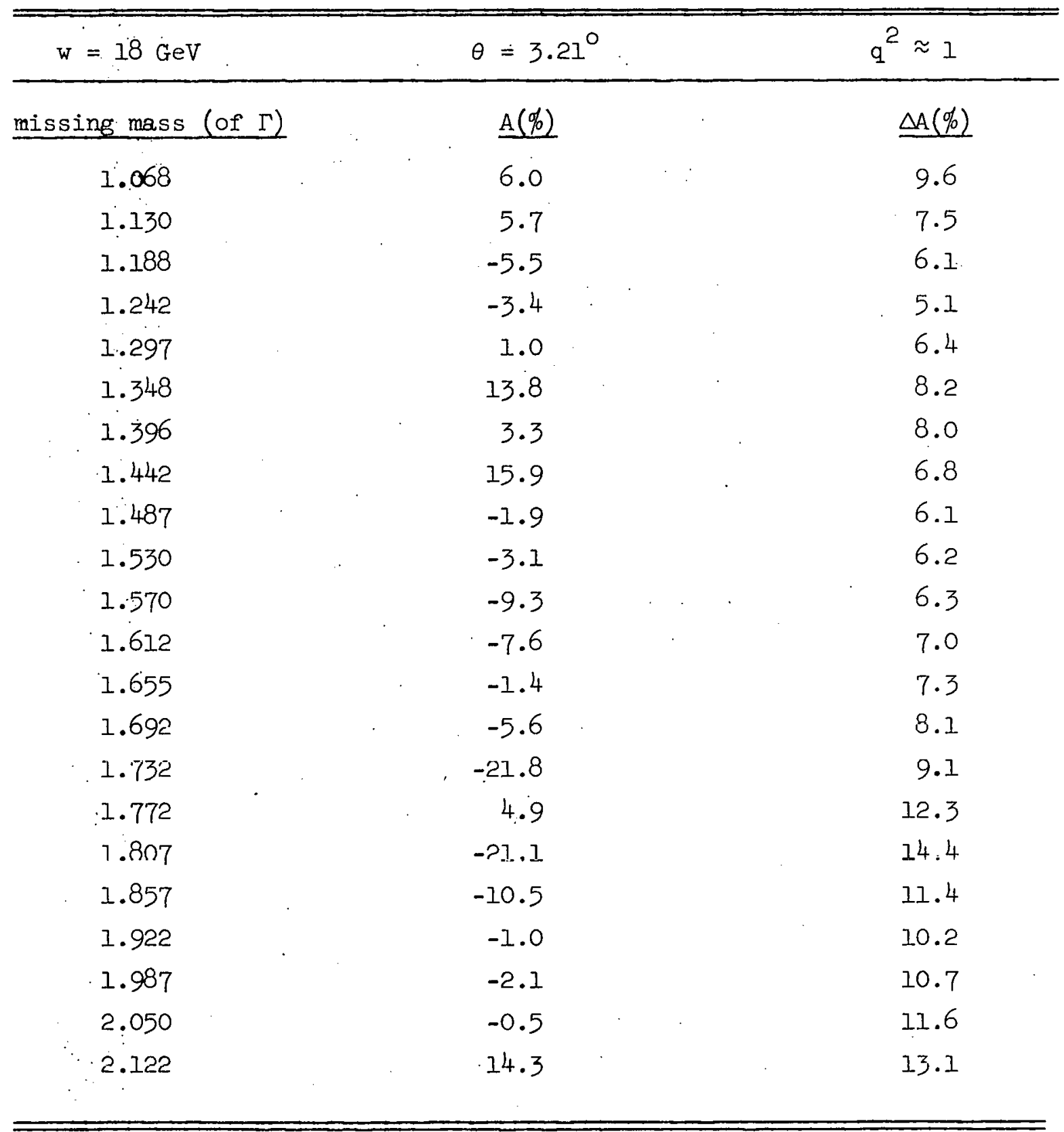




$$
-114-
$$

Table VIII. Asymmetry $A$ in $\mathrm{ep} \rightarrow \mathrm{e} \Gamma$ inelastic scattering. The error $\triangle \mathrm{A}$ is the statistical, error.

\begin{tabular}{|c|c|c|}
\hline $\mathrm{W}=12 \mathrm{GeV}$ & $\theta=3.18^{\circ}$ & $q^{2} \approx 0.4$ \\
\hline missing mass (of $\Gamma$ ) & $\mathrm{A}(\%)$ & $\triangle A(\%)$ \\
\hline 1.066 & 3.0 & 9.1 \\
\hline 1.106 & -8.3 & 6.6 \\
\hline 1.145 & -1.0 & 4.5 \\
\hline 1.182 & -6.8 & 3.5 \\
\hline 1.217 & -1.5 & 3.2 \\
\hline 1.252 & -4.5 & 3.7 \\
\hline 1.287 & 2.8 & 4.7 \\
\hline 1.322 & -9.2 & 5.2 \\
\hline 1.355 & 6.2 & 5.8 \\
\hline 1.387 & $7 \cdot 3$ & 7.6 \\
\hline 1.420 & -9.6 & 8.8 \\
\hline
\end{tabular}


Table IX. The percentage asymmetry values.(A) averaged over missing-mass bins corresponding to the resonances $\Delta(1236), N^{*}(1512)$ and $N^{*}(1688)$, using widths of $0.15,0.12$ and $0.11 \mathrm{GeV}$, respectively. In addition, a measurement in the deep inelastic region (mass $2.37-2.62 \mathrm{GeV})$, for $\mathrm{EO}=18.0 \mathrm{GeV}$ and $\mathrm{q}^{2}=0.54(\mathrm{GeV} / \mathrm{c})^{2}$, found $A=(-1.6 \pm 3.5) \%$. The data of Chen et al., (Ref. 10 are shown for comparison.

\begin{tabular}{|c|c|c|c|c|c|c|}
\hline \multirow{2}{*}{$\begin{array}{c}\text { Incident } \\
\text { beam }\end{array}$} & \multirow{2}{*}{$\begin{array}{l}\text { Incident } \\
\text { electron } \\
\text { energy, } \\
\mathrm{E}_{\mathrm{O}} \\
\mathrm{GeV}\end{array}$} & \multirow{2}{*}{$\begin{array}{l}\text { Four- } \\
\text { momentum } \\
\text { transfer } \\
\text { squared } q^{2} \\
(\mathrm{GeV} / \mathrm{c})^{2}\end{array}$} & \multicolumn{4}{|c|}{ Asymmetry value, $\mathrm{A}(\%)$} \\
\hline & & & $\Delta(1236)$ & $N^{*}(1512)$ & $\mathbb{N} *(1688)$ & \\
\hline $\mathrm{e}^{-} \quad \vdots$ & 18.0 & $0.58^{a}$ & $2.8 \pm 1.4$ & $-1 \cdot 3 \pm 1.7$ & $0.8 \pm 2.1$ & 量 \\
\hline$e^{+}$ & 12.0 & $0.42^{b}$ & $-3.0 \pm 1.8$ & -- & --- & $\mathbb{x}$ \\
\hline$e^{-}$ & 15.0 & $0.37^{a}$ & $2.3 \pm 2.9$ & $1.5 \pm 2.2$ & $1.2 \pm 3.1$ & . \\
\hline$e^{-}$ & 18.0 & $0.96^{a}$ & $-2.8 \pm 3.3$ & $-4.8 \pm 3.6$ & $-8 \cdot 2 \pm 4 \cdot 7$ & 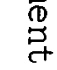 \\
\hline$e^{-}$ & 3.98 & $0.23^{b}$ & $3.8 \pm 4.3$ & - & -- & $\begin{array}{c}3 \\
\stackrel{5}{8}\end{array}$ \\
\hline$e^{-}$ & 5.97 & $0.72^{a}$ & -- & $3.6 \pm 4.7$ & $-0.5 \pm 4.4$ & 10 \\
\hline$e^{-}$ & 5.498 & $0.52^{a}$ & $-\cdots$ & $-2.6 \pm 8.2$ & $3.6 \pm 7.3$ & 1: \\
\hline
\end{tabular}

at $1.512 \mathrm{GeV}$ missing mass.

b At $1.236 \mathrm{GeV}$ missing mass. 
nance regions where a $\Delta I=0$ transition is possible $\left(N^{*}(1512), N^{*}(1688)\right)$ do not show any great resonance dominance and hence there are significant contributions from many partial waves. Thus the terms in the sum over all final states (all values of total angular momentum and different particle states) which we observe and which contribute to the form factors W(Eq. II-4) can cancel each other in the case of $\mathrm{W}_{3}$ and probably contribute in a random-walk fashion to a value small compared to the sum of the absolute values of the terms. On the other hand, the sums in $W_{1}$ and $W_{2}$ are just the sums of absolute values. The asymmetry, Eq. II-5, is therefore expected to be small (approximately $N(N)$ if each sum is dominated by $\mathrm{N}$ states, each of whose partial cross sections are comparable in magnitude. (It is important to distinguish between the sum just referred to which is forced on us by our apparatus with the sum over all orientations of the spin $\sum_{m_{j}}$ necessary to have 0 asymmetry in a T-conserving one- $\gamma$-exchange theory.)

(2) The transition between the initial proton, $I=1 / 2$, and the $N^{*}(1512)$ or $N^{*}(1688), I=1 / 2$ may be $\Delta I=0$ or 1 and there is no evidence as to which, or if both contribute. Any contribution from $\Delta I=1$ would reduce the expected asymmetry by Lee's $\Delta T=0$ rule.

(3) Even on the hypothesis of maximal T violation the phase between $F_{\text {_ }}$ and $F_{z}(\varphi)$ need not be $90^{\circ}$ since at least half the reaction amplitude is $\mathrm{T}$-conserving. Indeed Lee has pointed out ${ }^{41}$ that $\varphi \leq 10^{\circ}$ which would reduce our expected maximum asymmetry $A$ to $\leq 0.2 \times 30 \% \approx 6 \%$.

(4) The data on $\sigma_{I} / \sigma_{t}$ is quite poor in all cases and usually less than 2 S.D. from zero. $\sigma_{I}=0$ would mean $F_{z}=0$ and hence no asymmetry 
even if there is a T violation.

Despite these quite valid theoretical problems, it seems to me that we would be very unlücky to have seen no asymmetry over our entire range of kinematic parameters if there were a $\mathrm{T}$ violation in the electromagnetic interactions of hadrons. This experiment is thus evidence against (albeit far from conclusive) the hypothesis of Bernstein, Feinberg, and Lee.

Further research into the problem of the CP violation is necessary. It seems unlikely that a refinement of the experiment described in this report can be productively used to illuminate the mystery. Once the first theoretical estimates have been proved faulty, there is rarely a lower limit that theorists would not stoop to. Almost an order of magnitude reduction in experimental errors is conceivable by doubling the target polarization (by using a liquid $\mathrm{He}^{3}$ coolant) and collecting 10 times as many events using a crystal detector with large solid angle. (Between the conception and the act falls the shadow of untested technology.)

I believe that scarce resources would be better spent on other experimental approaches to the same CP violation. The $\eta \rightarrow \pi^{+} \pi^{-} \pi^{0}$ charge asymmetry has never been satisfactorily measured down to the limits of the initial theoretical estimates. Tests of possible $0.1 \% \mathrm{CP}$ violations in the strong and weak interactions are just reaching the required accuracy and should be encouraged. (For example, one may look for a Tviolating term in the beta decay of nuclei.) The measurement of $\eta_{0 O}$ must also receive priority. Why then redo once again the ep inelastic asymmetry just because possible techniques are available for experimental 


$$
-118-
$$

inprovement. Rather let the equipment be made to fit the potentially interesting experiment. 


\section{ACKNOWLIEDGMENTS}

I would like to thank God for not interfering in any major way with the successful operation of this experiment. Next in line is my thesis advisor Professor Owen Chamberlain, whose interference was a powerful influence on this experiment and my entire graduate career. The presence of a wise man is a rare opportunity to learn. His patience in reviewing this thesis is proof enough of his dedication as a teacher. Thanks also to Professor Gilbert Shapiro whose good ideas and advise were a grcat aid and to Professor Herbert Steiner whose spirit of hard. and careful work hung over the experiment even though he himself was absent during the actual running. The greatest effort and aid was given by my fellow graduate students, the now Dr. Tom Powell, trie now Dr. Charles Morehouse, the now Dr. Peter Robrish, and the future Drs. John Jaros and Steve Shannon. Their work and companionship were an important part of my graduate education. Ray Fuzesy, the outstanding master of all mechanical arts and operator of the polarized target at all hours deserves a special thanks, both for himself, and as representative of all the other skilled artisans who contributed to this experiment. I will never forget nor ever follow most of Ray's advice.

It was a pleasure to work with and learn from Richard Taylor, Les Cottrell, John Litt and Luke Mo of SLAC. Professor Taylor was a special personal guide. The skilled work and cooperation of the SLAC operating crew in obtaining a perfect beam for us deserves thanks.

My first child would like to thank my colleagues for the time off they gave to her father to enable her conception to tuke place. 


$$
-120-
$$

Private words to non Physics, extremely close associates, and my loving wife shall not be demeaned by being printed here.

I claim complete responsibility for the spelling in this report. Any errors are not due to Rosemary Fowell who worked so diligently attempting to turn chaos into typed order. 


\section{DFDTCATION}

I began my graduate student career amid the Free Speech Movement, began this experiment during the People's Park battles, and am writing this thesis amid a mass, nationwide, student strike to end the war in Vietnam. The old men of the physics world are still content to listen to government experts and to obey leaders they do not believe or respect, and they are afraid to voice their political opinions in public. Many are so grateful for government support of their research they will not oppose its wrongdoing. They still let the military decide what is the best way of using their research for the betterment of mankind. Even those who oppose the government act as private advisors and refuse to make public their advice and let the people decide.

If anything, my education has taught me the fallibility and greatness that man can attain, often simultaneously. Trust and faith in any collection of men can be entertaining and personally rewarding, but it is disastrous as a public means of running the country. I, as an expert in physics and a reader of many experts in other fields, know their limitless incompetence and equally limitless ability to hide such incompetence, which seems to be the only universal expertise. When the people make the cholce they may not always make the correct choice, but at least they know what the decision is and who is responsible. There is no reason to believe a government expert more than one who is outstide government. Unless we cease to be prisoners of those who pay our way, there will be no future. 
I pledge not to engage in any research which I consider to be destructive to man's physical or spiritual welfare and to counsel others to follow the same course of action. 


\section{APPENDIX A}

\section{Cancellatippiofigctuations}

In this section the manner in which the various types of random fluctuations cancel out due to target polarization reversals is discussed. Four cases will be dealt with. (I) The fluctuations vary slowly compared to target reversai frequency. (2) The fluctuations vary rapidly.

The fluctuations are delta functions. (4) The fluctuations are step functions.

Let us take a hypothetical situation of data acquisition with consecutive periods of data taking, each period with equal segments in which the target is polarized plus and minus. Time is measured by the number of beam particles, $q$, passing through the target and each of $\mathrm{K}$ periods has $2 Q$ particles ( $Q$ for each sign of polarization). Let $\mathbb{N}_{ \pm}$be the events/ period for each sign of polarization without any random fluctuations and let $f(q)$ be the events/(beam particle) which are due to the fluctuations. $\mathrm{T}=2 \mathrm{QK}$ is the total time of our hypothetical acquisition and $\mathrm{N}=\mathrm{K}\left(\mathrm{N}_{+}+\mathrm{N}_{-}\right)$ is the total number of counts in that time; both $T$ and $N$ are constant for a fixed duration of running. We wish to find values of $Q$ and $K$ to achieve maximum cancellation of random error. The asymmetry during the $i^{\text {th }}$ period of running is:

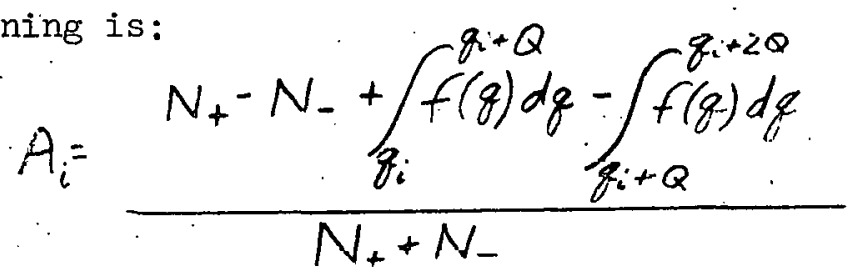

Averaging over the $\mathrm{K}$ periods:

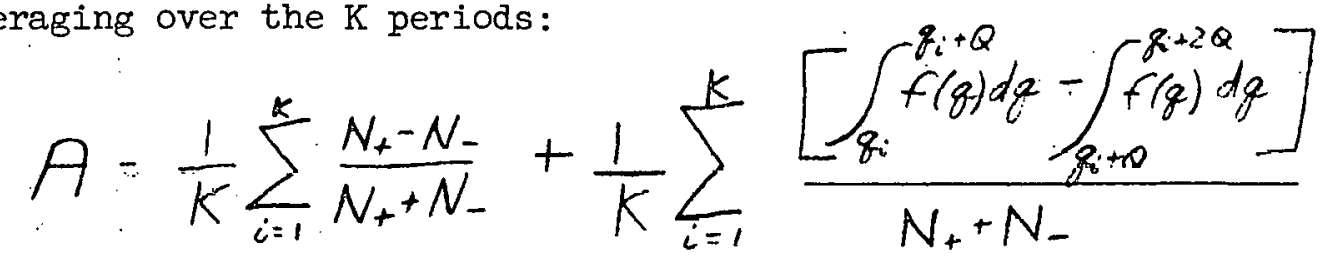




$$
-124-
$$

The second term is due to the fluctuations.

$$
A_{f l u c t}=\frac{1}{N} \sum_{i=1}^{K}\left[\int_{g_{i}}^{g_{i}+Q} f(q) d g-\int_{g_{i}+Q}^{g_{i}+2 Q}(q) d g\right] \quad(\beta-1)
$$

In case $(I) f(q)$ varies so slowly that the integrals in $(A-I)$ can be evaluated by replacing $f(q)$ by its value at the center of the interval and hence the difference becomes:

$$
f\left(q_{i}+Q / 2\right) Q-f\left(q_{i}+3 Q / 2\right) Q=-\left.\frac{d f}{d q_{i}}\right|_{i} Q^{2}
$$

The sum over all periods $i$ can be changed to an integral

$$
\sum \rightarrow \frac{1}{2 Q} \int \operatorname{dg}
$$

yielding:

$$
\begin{aligned}
A_{\text {fluct }} & =-\left.\frac{1}{N} \sum_{i=1}^{k} \frac{d f}{d q}\right|_{i} Q^{2}=-\frac{Q}{2 N} \int_{0} \frac{d f}{d q} d g \\
& =\frac{Q}{2 N}[f(0)-f(T)]=\frac{T[f(0)-f(T)]}{4 K N}
\end{aligned}
$$

All terms but $K$ depend only on the length of the total run. Hence the contribution of the random fluctuations to the asymmetry for a fixed data taking time is proportional to $I / K$ where $K$ is the number of target reversal cycles.

In case (2) $f(q)$ varies so rapidly that, on the average, it does not matter if we take the difference or sum of integrals in $(A-1)$. Using the sum we observe that the complete expression $(A-1)$ is just 


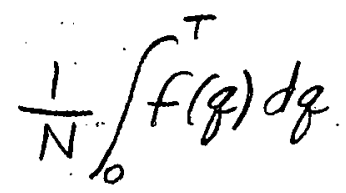

which depends only on the length of the data taking interval, and not on how we reverse the target.

In case (3) the integral (A-I) over the delta functions are again random numbers depending in magnitude on the size of the disturbance and depending in sign on the sign of the disturbance and on which of the two integrals (A-I).is involved. Thus, as in case (2), A fluct is independent of the manner in which the target polarization is reversed.

In case (4) first consider the effect of a single step of in $f(q)$ occurring at the time the polarization is changing from plus to minus in one of the terms in the sum $(A-1)$. Equation ( $A-1)$ then becomes:

$$
A_{\text {fluct }}=-(\delta f) \frac{Q}{N}=-\frac{T(\delta f)}{Z K N}
$$

If the etep occurred at another time, $A_{\text {fluct }}$ would be smaller and averaging over all possible times would introduce a numerical factor of if $\sqrt{3}$ into $(\mathrm{A}-2)$. Hence a step function fluctuation contributes to the asymmetry in a manner proportional to $1 / \mathrm{K}$ for a fixed interval of data taking. For many step functions, $m$ in number, the contribution from each one adds as in a random walk to give a resultant fluctuation proportional to $\sqrt{\mathrm{m}}$. Hence:

$$
A_{\text {fluct }}=\frac{\sqrt{m} T(\delta f)}{2 \sqrt{3} K N}
$$


Thus for a fixed $m$ the contribution of the fluctuation is proportional to $1 / \mathrm{K}$ for many random step functions in $\mathrm{f}(\mathrm{q})$. This last type of fluctuation is almost the same as case (I) since, if $m$ is very large and of small, $f(q)$ is almost a slowly varying continuous function. 
APPENDIX B

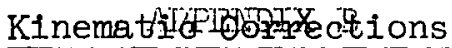

There are two types of kinematic corrections that must be made to the usual spectrometer optics due to the presence of our polarized target. (i) That due to the polarized target magnet and (2) that due to material in the beam.

(1) Since the magnet is at the target there is no significant focusing done by it. To correct for the bending of the beam and scattered particles by the magnet the relation $\theta=\theta_{\text {spec }}+\frac{1}{2} \theta_{\text {bend }}\left(1+w^{\prime} / w^{\prime}\right)$ is used. $\theta$ is the actual scattering angle, $\theta_{\text {spec }}$ the angle of the spectrometer with respect to the initial direction, $\theta_{\text {bend }}$ the angle which the beam bends in going through the entire magnet and $w$ and $\mathrm{w}^{\prime}$ are the beam and scattered electron energies. The first plus sign would be minus if the magnet were reversed to bend the beam toward the spectrometer. $\theta$ rather then $\theta_{\text {spec }}$ was used to calculate the actual missing mass.

(2) A minimally ionizing particle will lose about $5 \mathrm{MeV}$ traveling through our 2 gram $/ \mathrm{cm}^{2}$ target, with approximately equal energy loss before $(\Delta w)$ and after $\left(\Delta w^{\prime}\right)$ the interaction. Thus substituting $w \sim \Delta w$ and $w^{\prime}: \Delta w^{\prime}$. for the beam energy $w$ and detected energy $w^{\prime}$ will give us the actual missing mass $\left(M_{p}^{2}+2 M_{p}\left(w-\Delta w-w^{\prime}-\Delta w^{\prime}\right)-q^{2}\right)^{1 / 2}$. Using typical values of $\mathrm{w}=18.00 \mathrm{GeV}, \mathrm{w}^{\prime}=16.82 \mathrm{GeV}$ and $\mathrm{q}^{2}=0.58 \mathrm{GeV}^{2}$ this represents a shift of about $10 \mathrm{MeV}$. This correction, although small, is included in our kinematic calculations. 
APPENDIX C

\section{Target Heating E'tects}

The polarization obtainable with butanol targets is, near $1^{\circ} \mathrm{K}$, inversely proportional to the absolute temperature. 30 Thus it is important to keep the heat input low and the cooling efficient. The thermal conductivity of butanol is quite small $\left(0.5 \times 10^{-3} \text { watt/cm }-{ }^{\circ} \mathrm{K}\right)^{34}$ and so heat deposited in the interior of a block of butanol will raise its temperature considerably. This necessitates the small size of the ribs in the target containing bags.

Heat input to the target can come in the form of bulk (uniform) heating of the entire target or in the form of local heat input of large amounts to small volumes within the target. The former can be caused by the microwave power or by the loss of energy of the electron beam. Local heating could be caused by the beam and could cause total depolarization of small volumes within the target. Careful investigations were thus carried out to be certain that the beam was only causing bulk heating.

We could control the beam heating by controlling the beam intensity through the target and were thus able to measure the reaction of the target to heat loads. For bulk heating the expected relations are Polarization $\alpha i /$ Temperature and Temperature linearly dependent on beam intensity. This fits our data tor both $3 \mathrm{~mm}$ diameter bags $\left(\triangle \mathrm{P} / \mathrm{P}=-4.3 \% / 10^{\mathrm{I}} \mathrm{I}\right.$ electrons/. $\mathrm{sec})$ and $2 \mathrm{~mm}$ diameter bags $\left(\Delta \mathrm{P} / \mathrm{P}=-1.3 \% / 10^{11}\right.$ electrons $\left./ \mathrm{sec}\right)$ as shown in Fig. 26. Our use of $2 \mathrm{~mm}$ target containing bags was forced on us by these results.
A further confirmation of these beam bulk heating effects is seen 


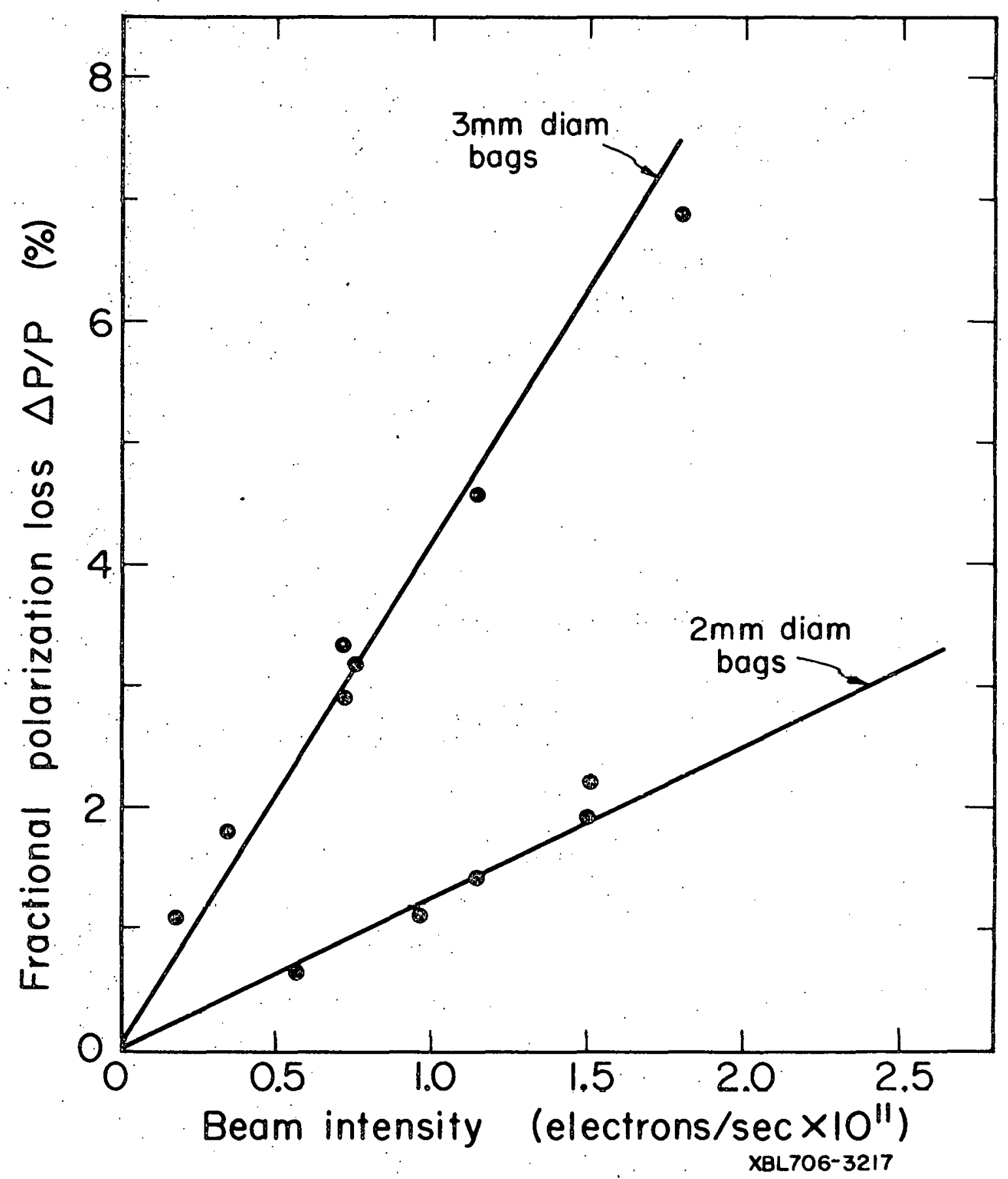

Fig. 26. Fractional polarization loss due to heating of the target by the beam. 
in the relaxation time (the time it takes the polarization to subside to 1/e of its initial value with microwave power off). The Raman process of electron relaxation, which is believed to be the mechanism through which our protons eventually relax, predicts a temperature dependence of $\mathrm{T}^{-7}$. This is completely consistent with our data: on comparison of Polarization ( $\alpha 1 / T)$ and relaxation time at various beam intensities. These effects discussed above are consistent with bulk heating of the entire sample. The possible effects of instantaneous local heating due to the small intense and short beam pulse depositing energy at the instantaneous rate of $10^{4}$ watts was investigated more completely. We wanted to prove that this local heating did not depolarize the small area the beam went through. Local depolarization would not be detected in our polarization measurements since only a tiny fraction of the target is involved. Stepping the beam after every pulse with the sweeping magnets was one built-in insurance against such a potential disáster. It was still important to investigate whether the first part of the beam might depolarize a spot and the remainder of the beam effectively pass through an unpolarized target. If the $\mathrm{T}^{-7}$ behavior continued up to $1 \cup^{\circ} \mathrm{K}$ (the approximate instantaneous raise in temperature) this might be a problem since the relaxation time at $1.05^{\circ} \mathrm{K}$ is about 300 seconds. We know of no mechanism is the target for relaxation in times as small as microseconds so this possibility is remote, but extremely dangerous. Two tests proved conclusively that the target was not locally depolarized.

(a) A small target, the size of the beam profile was installed. Local heating in this target was the same as bulk heating. We used a 
special polarization readout whose response was faster than the $1.6 \mu \mathrm{sec}$ beam pulse and thus would have been able to detect any instantaneous decline in polarization as the beam went through the target. None was observed.

(b) Normal Target. If we turn off the microwave power, then any depolarization will not be restored. Thus a comparison of relaxation time as a function of beam intensity (with the beam sweeping over the entire target so that each local volume was heated separately) would indicate any depolarization due to the beam. Local depolarization would have resulted in the target being completely depolarized after one complete beam sweep. The results are completely consistent with bulk heating with no local depolarization.

The effect of microwave power and resultant heating were also studied. The polarization increases with increasing microwave power until a maximum is reached and heating effects overcome increased spin flipping ability. We were willing to give up a little polarization magnitude to take advantage of the faster polarization reversal time which high microwave power offered. We estimate that 10 milliwatts $/ \mathrm{cm}^{3}$. was dissipated in the interior of our target (a total of about 0.25 watts) from microwave power. This is over $60 \%$ of the total microwave power dissipated in the cryostat. 
APPENDIX D

\section{Statistical Independence of Test Asymmetries}

The condition for statistical independence of two different patterns of pretended target reversal is that there should be as many intervals when the pretended polarizations are the same as when they are different. This condition is derived from the transformation of the error matrix, Wab, from one set of variables, $x$, to another, $x^{\prime}$,

$$
V_{i j}^{\prime}=\sum_{\mu \nu} \frac{\partial x_{i}^{\prime}}{\partial x_{\mu}} \frac{\partial x_{j}^{\prime}}{\partial x_{\nu}} V_{\mu \nu} .
$$

$x_{\mu}$ will be taken to be the number of counts in a subrun, $N_{\mu}$, while one of the other set of variables, $\dot{x}_{j}^{\prime}$, will be the observed test asymmetry in pattern $j$. Thus $x_{j}^{\prime}=x_{j}^{\prime}\left(N_{I}, N_{2}, \ldots N_{n}\right)$. In fact, from Eq. VII-3

$$
X_{j}^{\prime}=\left[\sum_{j^{+}} N_{\mu}-\sum_{j=} N_{\mu}\right] /\left[\sum_{j^{+}} N_{\mu}+\sum_{j=} N_{\mu}\right]
$$

where $j^{+}\left(j^{-}\right)$represent sums over all subruns $\mu$ for which the polarization is considered positive (negative) for asymmetry pattern. $j$. Since the $\mathbb{N}_{\mu}$, being counts in separate subruns are statistically independent of each other the error matrix elements are

$$
V_{\mu 2}=\left(\Delta x_{\mu}\right)^{2} \delta_{\mu 2}=N_{\mu} \delta_{\mu 2}
$$

where we have used normal counting statistics for the errors. In our case the observed asymmetry $\epsilon$ is $<2 \%$ because of the dilution factor $\mathrm{H}_{f} \mathrm{P}$ and hence the number of counts/subrun is a constant, $\mathrm{N}$, to better 
than $4 \%$ and $v_{\mu \dot{\nu}}=N_{\dot{\mu} \dot{y}}$ Now:

$$
\frac{\partial x_{j}^{\prime}}{\partial N_{\mu}}=\frac{(-1)^{j(\mu)}}{\sum N_{\lambda}}-\frac{\varepsilon}{\left(\sum N_{\lambda}\right)^{2}}
$$

where the second term is negligibly small since $\epsilon \leq 0.02$ and $\sum N_{i}=$ total counts in pattern is several thousand. $j(\mu)$ is $O(1)$ when $\mu$ is in $j^{+}\left(j^{-}\right)$. Hence:

$$
V_{R j}^{\prime}=\sum_{\mu=2} \frac{(-1)^{j(\mu)+k(\mu)}}{\left(\sum N_{\lambda}\right)^{2}} N \delta_{\mu \nu}=\text { constants } \times \sum_{\mu c}(-1)^{j(\mu)+k\left(\mu_{0}\right)}
$$

Thus if $k$ and $j$ are two patterns in which there are as many subruns in which the pretended polarization are the same $(j(\mu)+k(\mu)$ even) as subruns when they are opposite $(j(\mu)+k(\mu)$ odd) the sum cancels itself out to zero and the two patterns are statistically independent of each other (no correlation). This was the criterion used to make up these patterns. 


\section{APPENDIX E}

\section{Deadtime}

Here I will show that a rate-dependent deadtime can introduce an asymmetry if there is an asymmetry in the beam intensity. An asymmetry in the beam intensity (higher intensity during one sign of polarization then the other) will lead to an asymmetry in the event rate (events/second) and hence to an asymmetry in the amount of deadtime (more deadtime during one sign of polarization then the other).

Let $\mathrm{D}_{+}$and $\mathrm{D}_{-}$be the fraction of counts lost during + and - polarization due to deadtime and $\mathrm{N}_{ \pm}$be the measured counts. Then:

$$
\begin{aligned}
P H_{f} A_{\text {actual }} & =\frac{N_{+}\left(1+D_{+}\right)-N_{-}\left(1+D_{-}\right)}{N_{+}\left(1+D_{+}\right)+N_{-}\left(1+D_{-}\right)} \\
& =\frac{N_{+}-N_{-}}{N_{+}+N_{-}+N_{+} D_{+}+N_{+} D_{-}}+\frac{N_{+} D_{+}-N_{-} D_{-}}{N_{+}+N_{-}+N_{+} D_{+}+N_{-} D_{-}}
\end{aligned}
$$

For small asymmetries (as in this experiment) $\mathbb{N}_{+} \approx \mathbb{N}_{-} \approx \mathbb{N}$

$$
P H_{f} A_{\text {a.ctual }}=\frac{N_{+}-N_{-}}{N_{+}+N_{-}+N\left(D_{+}+D_{-}\right)}+\frac{N\left(D_{+}-D_{-}\right)}{N\left(Z+D_{+}+D_{-}\right)}
$$

For small deadtimes $D_{+}, D_{-} \ll I_{1}$. Hence:

$$
\begin{aligned}
\text { PH } H_{f} A_{\text {acrual }} & =\frac{N_{+}-N_{-}}{N_{+}+N_{-}}+\frac{D_{+}-D_{-}}{2\left(D_{+}+D_{-}\right)} \cdot\left(D_{+}+D_{-}\right) \\
& =P H_{+} A_{\text {measured }}+A_{d} D
\end{aligned}
$$

where $D=\left(D_{+}+D_{-}\right) / 2$ and $A_{d}=\left(D_{+}-D_{-}\right) /\left(D_{+}+D_{-}\right)$is the asymmetry of the deadtime which is the same as the asymmetry of the beam intensity. Thus If both $A_{d}$ and $D$ are nonzero a nonzero asymmetry can be introduced into our results. 
APPENDIX F

The Phony Bump

This is a discussion of the apparent nonzero asymmetry in our data at $\mathrm{q}^{2}=0.6$ centered at missing mass of $1200 \mathrm{MeV}$. The average asymmetry over the three points is $(4.5 \pm 1.4) \%$. This 3.2 S.D. asymmetry has a $0.137 \%$ chance of occurring in this particular spot. However since our real data contains 107 bins there are a total of 105 adjacent sets of 3 bins which gives a $105 \times 0.137 \%=15 \%$ statistical chance for this deviation to appear somewhere in our real data. This is the equivalent to considerably less then 2 S.D. This analysis is confirmed by generating random asymmetries using a random number generator to simulate the counts in our experiment. Three out to 25 sets of asymmetry spectra show fluctuations of the magnitude of our actual real data, for a $12 \%$ probability. Analysis of our test asymmetries shows the probability to be $30 \%$. Hence this data can be completely explained as a quite probable statistical fluctuation once it is realized the large quantity of data we have. Perhaps the moral is that the more data one takes, the less significant each part of it might be unless one knows the physics behind the process well enough not to dilute data taken where a real effect might occur 'with other data taken where a real effect will not occur.' We believe the argument above to be sound as it stands, but we are nevertheless inclined to point out that the region of missing mass around $1200 \mathrm{MeV}$ is a particularly unlikely place for a real nonzero asymmetry to appear. This 'is a consequence of the $\Delta I=0$ rule. A 2- $\gamma$-exchange-caused asymmetry is extremely unlikely, due to the large size of the asymmetry 
compared to the calculations of Tsai and Cahn, ${ }^{12}$ who predict $0.6 \%$. A large 2-y effect at this missing mass would be hard to reconcile with no observable 2- $\gamma$-exchange asymmetry in elastic scattering ${ }^{14}$ at almost the same kinematic conditions. Thus, far from diluting the $\mathrm{q}^{2}=0.6$, missing mass $=1200 \mathrm{MeV}$, data, the rest of the experimental results are expected to be more significant, hence reinforcing our interpretation of this small asymmetry as a statistical fluctuation. 


\section{REFERENCES}

1. A. Wightman and R. Streater, PCT, Spin and Statistics, and all that, (W. A. Benjamin, New York, 1964).

2. J. Steinberger, Review of the experimental situation relevant to $\mathrm{CP}$ violation, in Topical Conference on Weak Interactions, CERN, Jan. 1969. (CERN, Geneva, 1969).

3. T. D. Lee and C. N. Yang, Phys. Rev. 104, 254 (1956).

4. C. s. Wu, et al., Phys. Rev. 105, 1413 (1957).

5. J. H. Christenson, et al., Phys. Rev. Letters 13, 138 (1964).

6. J.: Bèrnstein, G. Feinberg, and T. D. Lee, Phys. Rev. 139, B1650 (1965), see also:

T: D. Lee, Phys. Rev. 140, B967 (1965);

T. D. Lee, Pliys. Rev. 140, B959 (1965);

T. D. Lee, Proceedings of $13^{\text {th }}$ International Conference on High Energy Physics (University of California Press, Berkeley, 1967); T. D. Lee, Physics Today, Vol. 19, No. 3 (1966);

T. D. Lee, Proceedings of the 1967 International Symposium on Electron and Photon Interactions at High Energies, Stanford Linear Accelerator Center (AEC, 1967) p. 389;

N. Christ, Possible Tests of $\mathrm{C}_{\mathrm{ST}}$ and $\mathrm{T}_{\mathrm{ST}}$ Invariance of Electromagnetic Phenomena, (thesis), Columbia University, 1966.

7. T. D. Lee, Phys. Rev. 139, B1415 (1965);

J. Bernstein, G. Feinberg, and T. D. Lee, IPlys R. Rev. (1395) Bl650 (1965).

8. M. Gormley, et al., Phy's. Rev' Letter's 2l, 399 (1968).

9. N. Christ and T. D. Lee, Phys. Rev. 143, Bl310 (1966). 
10. J. R. Chen, et al., Phys. Rev. Letters 로, 1279 (1968).

11. J. D. Bjorken and J. D. Walecka, Electroproduction of Resonances, SLAC-PUB-139.

12. R. Cahn and Y. S. Tsai, SLAC-PUB-722.

13. E. D. Bloom, D. H. Coward, H. De Staebler, J. Drees, L. W. Mo, R. E. Taylor, M. Breidenbach, J. I. Friedman, and H. W. Kendall, Phys. Rev. Letters 23, 930 (1969).

14. Thomas Powell, Michel Borghini, Owen Chamberlain, Raymond Z. Fuzesy, Charles C. Morehouse, Stephen Rock, Gilbert, Shapi,ro, Howard Weisberg, Roger L. A. Cottrell, John Litt, Luke Mo, and Richard E. Taylor, Phys. Rev. Letters 24, 753 (1970.

15. See for example: L. N. Hand, Phys. Rev. 129, 1834 (1963).

16. T. D. Lee, Phys. Rec. 140, B959 (1965);

T. D. Lee, Proceedings of the 1967 International Symposium on Electron and Photon Interactions at High Energies, Stanford Linear Accelerator Center (AEC 1967), p. 389.

17. L. B. Okun, Physics Letters 23, 595 (1966).

18. L. B. Okun and C. Rubbia, Proceedings of the Heidelberg International Conference on Elementary Particles (North-Holland Publishing Co., Amsterdam, 1968) footnote p. 336.

19. A. A. Cone et al., Phys. Rev. 156, 1490 (1967);

H. L. Lynch, J. V. Allaby, and D. M. Ritson, Phys. Rev. 164, 1635 (1967);

F. W. Brasse et al., Nuovo Cimento 55A, 679 (1968);

W. Bartel et al., Phys. Letters 27B, 660 (1968). 
W. Albrecht et al., DESY-69/7 (Deutsches Elektronen-Synchrotron, Hamburgi).

20. A. A. Cone et al., Phys. Rev. 156, 1490 (1967).

21. N. Christ and T. D. Lee, Phys. Rev. 148, 1520 (1966).

22. R. S. Larsen and D. Horelick, Report No. SLAC-PUB-398, Stanford Linear Accelerator Center (1968).

23. G. E. Fischer and Y. Murata, Report No. SLAC-PUB-605, Stanford Linear Accelerator Center (1969), (to be published in Nucl. Instr. and Methods).

24. K. B. Mallory et al., Instrumentation and Control, Chap. 15 in The Stanford Two-Mile Accelerator, edited by R. B. Neal (W. A. Benjamin, N. Y., 1968).

25. SLAC User's Handbook, Section E, Stanford Linear Accelerator Center; and W.K.H. Panofsky, Proceedings of the International Symposium on Electron and Photon Interactions at High Energies, Hamburg, Germany (1965).

26. A. Boyarski, "The online control, analysis and display system for the SLAC multi-GeV/c spectrometers, "Proceedings of the Conference on Computer Systems in Experimental Physics, Skytop, Penn., (March 1969).

27. M. Borghini, Polarized Proton Targets, Ecole Internationale de La Physique des Particules Elementaires, Herceg Novi (Yougoslavie) 1968;

C. D. Jeffries, Dynamic Nuclear Orientation (Interscience Publishers, Inc., New York, 1963). 
$-140-$

28. V. K. Ermolaev, Yu N. Molin and N. Ya Buben, Kinetika i Katiliz 3, 58 (1962), English translation Kinetics and Catalysis 3, 46 (1962).

29. Claude Schultz, Scattering of $250-\mathrm{MeV}$ Positive Pi Mesons from a Polarized Proton Target, (thesis) UCRL-11149.

30. S. Mango, 0. Rwnofsson, and M. Borghini, Nucl. Instr. Methods 72 , $4.5(1.969)$;

Michel Borghini, Owen Chamberlain, Raymond Z. Fuzesy, Willlam Gorr, Charles C. Morehouse, Thomas Powell, Peter Robrish, Stephen Rock, Stephen Shannon, Gilbert Shapiro, and Howard Weisberg, Nucl. Instr. and Methods (to be published), (UCRL-19724, March 1970).

32. J. R. Chen, et al., Phys. Rev. Letters 21, 1279 (1968);

H. Weisberg, et al., Bull. Am. Phys. Soc. 13, 164 (1968).

33. Private communication. Raymond Z. Fuzesy.

31. Michel Borghini, ot al., Nucl. Instx. and Methods (t.n he published), (UCRL-19724, March 1970).

35. Fluorinated Ethylene-Propylene film, type A, 0.0005 inch thick. Fluorocarbon Corp., Pine Brook, New Jersey.

36. A. Masaike, et al., Phys. Letters 30A, 63 (1969).

37. P. Roubeau, Cryogenics 6, 207 (1966), and thesis (Grenoble, 1966).

38. Q. A. Kerns, H. W. Miller, and A. K. Wolverton, UCRL-17548.

39. Charles C. Morehouse, and friends, UCRL-19897.

40. Paul Grannis, Measurement of the Polarization Parameter in ProtonProton Scattering from 1.7 to $6.1 \mathrm{BeV}$, (thesis) UCRL-16070 (1965).

41. T. D. Lee (Columbia University), private communication to Gilbert Shapiro, April 1970. 


\section{LEGAL NOTICE}

This report was prepared as an account of Government sponsored work. Neither the United States, nor the Commission, nor any person acting on behalf of the Commission:

A. Makes any warranty or representation, expressed or implied, with respect to the accuracy, completeness, or usefulness of the information contained in this report, or that the use of any information, apparatus, method, or process disclosed in this report may not infringe privately owned rights; or

B. Assumes any liabilities with respect to the use of, or for damages resulting from the use of any information, apparatus, method, or process disclosed in this report.

As used in the above, "person acting on behalf of the Commission" includes any employee or contractor of the Commission, or employee of such contractor, to the extent that such employee or contractor of the Commission, or employee of such contractor prepares, disseminates, or provides access to, any information pursuant to his employment or contract with the Commission, or his employment with such contractor. 
TECHNICAL INFORMATION DIVISION

LAWRENCE RADIATION LABORATORY

UNIVERSITY OF CALIFORNIA

BERKELEY, CALIFORNIA 94720 